\title{
DOE/mc/31173 -.24
}

\section{Industrial Advanced Turbine Systems: Development and Demonstration}

\author{
Annual Report \\ October 1, 1996 - September 30, 1997
}

Work Performed Under Contract No.: DE-FC21-95MC31173

For

U.S. Department of Energy

Office of Fossil Energy

Federal Energy Technology Center

P.O. Box 880

Morgantown, West Virginia 26507-0880

By

Solar Turbines

2200 Pacific Highway

PO Box 85376

San Diego, California 92186-5376

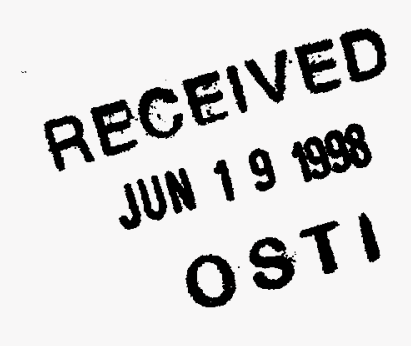




\section{Disclaimer}

This report was prepared as an account of work sponsored by an agency of the United States Government. Neither the United States Government nor any agency thereof, nor any of their employees, makes any warranty, express or implied, or assumes any legal liability or responsibility for the accuracy, completeness, or usefulness of any information, apparatus, product, or process disclosed, or represents that its use would not infringe privately owned rights. Reference herein to any specific commercial product, process, or service by trade name, trademark, manufacturer, or otherwise does not necessarily constitute or imply its endorsement, recommendation, or favoring by the United States Government or any agency thereof. The views and opinions of authors expressed herein do not necessarily state or reflect those of the United States Government or any agency thereof. 


\section{DISCLAIMER}

Portions of this document may be illegible electronic image products. Images are produced from the best available original document. 


\subsection{INTRODUCTION}

The U.S. Department of Energy (DOE) has initiated a program for advanced turbine systems (ATS) that will serve industrial power generation markets. The objective of the cooperative agreements granted under the program is to join the DOE with industry in research and development that will lead to commercial offerings in the private sector. The ATS will provide ultra-high efficiency, environmental superiority, and cost competitiveness. The ATS will foster (1) early market penetration that enhances the global competitiveness of U.S. industry, (2) public health benefits resulting from reduced exhaust gas emissions of target pollutants, (3) reduced cost of power used in the energy-intensive industrial marketplace and (4) the retention and expansion of the skilled U.S. technology base required for the design, development and maintenance of state-of-the-art advanced turbine products.

The Industrial ATS Development and Demonstration program is a multi-phased effort. Solar Turbines Incorporated (Solar) has participated in Phases 1 and 2 of the program. On September 14, 1995 Solar was awarded a Cooperative Agreement for Phases 3 and 4 of the program (DE-FC2195MC31173) by the DOE's Office of Energy Efficiency and Renewable Energy (EE). Technical administration of the Cooperative Agreement will be provided from EE's Chicago Operations Office. Contract administration of the Cooperative Agreement will be provided from DOE's Office of Fossil Energy, Federal Energy Technology Center (FETC).

Phase 3 of the work is separated into two subphases: Phase $3 \mathrm{~A}$ entails Component Design and Development; Phase 3B will involve Integrated Subsystem Testing. Phase 4 will cover Host Site Testing. The cooperative agreement funding is separated into three budget periods. Budget Period 1 expires March 14, 1998.

\subsection{SUMMARY PROGRAM ASSESSMENT}

\subsection{Estimate at Completion}

Forecasts call for completion of the program within budget as originally estimated. Scheduled completion is forecasted to be approximately 3 years late to original plan. This delay has been intentionally planned in order to better match program tasks to the anticipated availability of DOE funds. To ensure the timely realization of DOE/Solar program goals, the development schedule for the smaller system (Mercury 50) and enabling technologies has been maintained, and commissioning of the field test unit is scheduled for May of 2000 . The development schedule for the larger system (ATS-L) has been delayed to accommodate the funding shortfall without undue impact to near-term program goals, with commissioning of the field test unit planned for March of 2002.

Significant efforts have been spent since mid-year to reforecast and control expenditures due to Solar's and DOE's current funding and resource constraints. Selective reductions and delays in program activities were identified and implemented. Although these actions will increase technical risk and the attainment of stretch goals, it is not anticipated that the schedule for initial test units or the attainment of basic program performance requirements will be impacted.

\subsection{Overall Assessment of Performance}

As of the end of the reporting period work on the program is $22.80 \%$ complete based upon 
milestones completed. This measurement is considered quite conservative as numerous drawings on the Mercury 50 are near release. (In order to main objectivity in assessing schedule progress, Solar uses a 0/100 percent complete assumption for milestones rather than subjectively estimating progress toward completion of milestones.) Variance information is provided in Section 4.0 Program Management.

Mercury 50: Work this year has focused on detailed design and the release of long-lead, critical part drawings. As is usually the case with programs involving any major turbine design, stages 1 and 2 blades and nozzles are pacing items and are consuming a large portion of the design resource.

Major design reviews held this year include those listed in Table 1. Each of the reviews resulted in positive suggestions for design improvements (mechanical integrity or cost); none were sufficient to impact the engine schedule.

Good progress has been made on the release of compressor parts during this reporting period, there being only two remaining stages of blades and disks left to release to the suppliers. The aft hub is the only remaining critical compressor part of significance.

The latest estimate of engine drawing completion is $45-50 \%$.

\begin{tabular}{|l|l|}
\hline \multicolumn{1}{|c|}{ Subassembly /Component } & \multicolumn{1}{c|}{ Date } \\
\hline Stage 2 Nozzle and Diaphragm Assembly & 7 March \\
\hline Preswirl Assembly & 7 April \\
\hline Exhaust Collector & 8 April \\
\hline Stage 2 Blade & 15 April \\
\hline Combustor End Cover & 17 April. 9 May \\
\hline Center Frame Assembly & 3 May \\
\hline Nozzle Case Assembly & 6 May \\
\hline Combustion System Assembly & 9 May \\
\hline Fuel Injector Assembly & 9 May \\
\hline Stage 1 Blade and Disk & 15 August \\
\hline Stage 1 Nozzle & 27 August \\
\hline Catalytic Combustor & 05 Sept \\
\hline
\end{tabular}

Table 1. Mercury 50 Design Reviews

Over 200 preliminary drawings were issued over the past 12 months. In order to maintain an aggressive schedule, Solar design engineers, manufacturing engineers and suppliers work 
concurrently based upon various levels of controlled preliminary drawings (CPDs) rather than working in serial manner based on the formal release of final drawings. Also some 160 tools are now order to support engine and rig build dates.

Solar received three development gear box housings this quarter, which were machined and delivered to assembly at Kearny Mesa. Kearny Mesa Assembly has completed the assembly of one gear box and delivered it to Superior Gear for test.

Twelve compressor case casting halves have been received and are awaiting the machining design release. The first development nozzle support forging was received, which will be used for machining development at the supplier. This first part is a Haynes 242 alloy and will be reserved for future testing.

Howmet is building casting tools for the first stage nozzle, second stage nozzle, first stage blade and second stage blade. Texas Steel built the patterns for the combustor end cover and the forward and aft end plates on the center frame. Many castings and forgings are scheduled to arrive during the next quarter.

Rigs and Technology Development: Work on the test rigs and in the enabling technology development programs is also progressing well. Significant milestones during the year have included:

- The design, assembly, installation, instrumentation and running of the compressor test rig at Wright Patterson Air Force Base. The rig was designed and constructed by The Agilis Group. Test results demonstrated compressor efficiency more than two points better than state-of-the-art industrial compressors.

- The designed, fabrication, instrumentation, assembly and running of the squeeze film damper rig in support of the Mercury 50 gas turbine anti-friction bearing system. Baseline testing using an existing damper design was completed; follow-on configurational option tests using a new Solar design is scheduled in early 1998.

- The design, fabrication and commencement of test activities on the half scale, uncooled. two and a half stage turbine aerodynamic test rig. The stage 1 nozzle flow tests have been completed, and baseline testing will commence in the upcoming reporting period.

- The completion of $f \& j$ testing (combined and gas-side core) on the Caterpillar Technology Center transient rig, and the fabrication, delivery and installation of a 100 cell performance core at the Caterpillar Technology Center, to measure the thermal effectiveness to establish the heat transfer and pressure drop characteristics of the Mercury 50 primary surface recuperator (PSR).

- Completion of testing on thermally painted screw cooled blades in Mars test engine confirming the effectiveness of this advanced airfoil cooling design to reduce blade leading edge temperatures while using less cooling air flow.

- Completion of single can catalytic combustion tests, demonstrating successful startup and transition from part load to catalytic operation, with NOx emissions consistently below 5 ppmv in catalytic operation at full load. 
- The completion of design of the ultra-lean premix annular combustor rig and the construction of the housing and endcover, as well as the assembly of the first injector, for the rig. Atmospheric testing on the rig is scheduled to commence in the upcoming reporting period.

- Completion of a 2000 hour test of thermal barrier coatings (TBC) on Stage 1 turbine blades in a Mars field engine and a 50 hour test of TBC on a Centaur 50 combustor liner with no degradation or spallation of TBC observed.

- Successful forging, heat treating and mechanical property testing of subscale Udimet 720 turbine disks for the first two engines. Test results exceeded creep and rupture life targets.

- Installation and commencement of testing of the 4000 hour Ceramic Stationary Gas Turbine (CSGT) field test engine at the ARCO Bakersfield site. The successful startup of this engine under the CSGT contract marks the first ever operation of a gas turbine incorporating ceramic components in an industrial application environment. The modified Centaur 50 gas turbine contains AS-800 silicon nitride Stage 1 turbine blades fabricated by Allied Signal Ceramic Components and inner and outer SIC/SIC CFCC combustor liner fabricated by Dupont Lanxide Corporation.

- Construction of the vortex cooling heat transfer rig at University of Utah to evaluate heat transfer coefficients.

- The completion of vacuum die cast process and the receipt of test bars for material testing.

- The modification of $347 \mathrm{SS}$ to produce a potential recuperator material having two to four times the creep life of standard 347 SS. A patent disclosure on the process has been initiated.

ATS-L: Work during the past year focused upon concept design. The Product requirements specification was completed, and the Business Plan was approved, in the second quarter 1997. An engine concept design review was held in July. Preliminary design proceeded with considerable effort spent on investigating alternative combustor designs. A preliminary design review is anticipated by year end.

Commercialization: Solar completed its ATS Commercialization Plan detailing the required efforts to promote distributed generation appropriately shape the ATS product and communicate the applicability of ATS products to fit these needs. The Plan was submitted to DOE in March 1997. The following subcontracted studies and reports were included as appendices to the Plan:

- Global Market Survey Report prepared by Duke/Fluor Daniel;

- Energy Use in the Industries of the Future prepared by Onsit Energy Corporation; and

- Environmental, Energy and Social Issues Affecting Distributed Generation Siting prepared by Resource Catalysts. 
The latter study was funded with matching funds provided by the California Trade and Commerce Agency, Office of Strategic Technologies.

A full time Mercury 50 Sales and Marketing Manager was appointed in mid-April reporting to Solar's Director of Worldwide Industrial Power Generation (IPG) Sales. The Mercury 50 product was formally announced at the American Society of Mechanical Engineers (ASME) Turbo Exhibition in Orlando, Florida the first week in June. A Mercury 50 data sheet was released two weeks later at PowerGen Europe in Madrid.

Meetings were held in Rochelle, Illinois and at Solar's San Diego facilities during the Third quarter to pursue locating the Mercury 50 field demonstration unit within the Rochelle Municipal Utilities' service district. Formal announcement of the host site is anticipated in December.

Detailed highlights from the most recent quarter are discussed below.

\subsection{TECHNICAL PROGRESS BY TASK}

\subsection{Mercury 50 Engine and Package}

\section{WBS 10200: Engine Design}

Task complete.

\section{WBS 10300: Package Definition}

A design review was held in early September to status the package design effort and to ensure that the overall system schedule will be supported. As of that meeting the following subjective estimates of completion were made: frame design - $60 \%$; recuperator support structure - $25 \%$; lube oil system - 75\%; gas fuel system - 50\%; air assist system - 50\%; and electrical packaging $25 \%$.

This quarter saw comprehensive development of detail design for the package with a focus on engine assembly removal from the forward end. The air inlet duct and the relief valve system design were completed.

\section{WBS 11100: Air Inlet System}

The inlet muff drawing has been fully reviewed with the supplier (Ketema) during this reporting period. Several minor changes have been requested to simplify the fabrication of this part. After a number of aerodynamic CFD (computational fluid dynamics) iterations based on preferences from Package Design, the air inlet duct configuration was accepted by all parties concerned, with minimal pressure loss compared with original designs.

\section{WBS 11200: Compressor Assembly-Mercury 50}

Compressor Static Structure: Detail design of static hardware has progressed well during this 
reporting period.

Preliminary machining drawings for the air inlet housing, compressor case and compressor discharge were issued for hardware procurement. \#1, \#2, and \#3 bearing housing casting drawings were also issued and parts were ordered. Some changes to the datum system have been requested by Manufacturing relating to the compressor discharge machining drawing. These changes will be made, and the drawing will be reissued.

The inner diffuser assembly has been issued to allow fabrication of this part. The outer diffuser assembly is also being detailed. Oil seals for the \#1 bearing housing have been issued.

The outlet guide vane (OGV) went through a couple of major iterations during this reporting period. Product cost considerations played an important role in the final design selection. The inner diffuser casting and the semi-machined stator ring were redesigned accordingly. Changed castings will support the first few engines with extra machine time required.

Casting drawings for the IGV, VGV1, and VGV2 have been issued for part procurement. The stator vane 6 drawing has been issued for tooling. Stator vane design is complete for stages 7 and 8 , and drawings are in the process of being created at Agilis.

Variable Guide Vane Actuation System: During this reporting period the layout of the vane actuation system has been completed. Drawings for the IGV, 1st stage and 2nd stage vane actuation rings were issued to initiate tooling. Two of the three rings have been designed similar to existing Solar engines, and the 2nd stage will be manufactured using tooling common with the IGV and 1st stage rings. No new tooling will be required for any of the rings. The supplier has reviewed the design and given feedback, which facilitated manufacturing processes.

Prototype components for the new variable vane shaft design were fabricated by the current vane supplier and are in transit to Solar. Availability of prototype test pieces will speed up the release of machined vane drawings.

Casting drawings for the IGV inner guide vane ring have been issued. The interface with the air inlet housing, the 1st stage compressor disk, and the inlet guide vane have been worked sufficiently to allow the supplier to proceed with the machining of the inner ring. Sufficient latitude has been incorporated in the design to allow for future potential changes without having to delay component delivery.

Compressor Rotor: Issuance of blade drawings progressed well during this reporting period. Drawings and electronic data for compressor blades 1 and 2 have been issued to the new forging supplier. Blades 3,4 , and 5 castings were received, and the first article inspections were completed for these blades. Some minor issues were evident relating to the chord width and platform contour profile; however the airfoils themselves were excellent in quality. The first metal samples of blade 6 castings were completed. Casting drawings of blades 7 and 8 were recently issued to the supplier. The machining supplier was recently chosen for blades 3 through 10.

Disk and spacer drawings were also worked vigorously during the last reporting period. Disk assembly drawings for tooling purposes were issued for stages 1 through 9 . The forward and aft hub semi-machined drawings were completed and issued. 


\section{WBS 11300: Combustor Assembly}

Combustor Case: The endcover castings were received from Texas-Steel. These two castings will be used for the combustor rig testing. Also, the combustor housing casting drawing was released to Wisconsin Centrifugal enabling tooling fabrication.

A visit was made to the machining supplier, Major Machining, to review the endcover and housing machining drawings in detail. Many suggestions were made based on Major's experience with Solar parts. Most of these suggestions have been incorporated into the drawings.

A material test plan has also been conceptualized. This will involve testing 347 stainless steel keel bars for creep and fatigue properties. Test samples will then be removed from an endcover casting. These will be correlated to the keel bars. These tests will be used to verify design margins.

A 3D elastic/plastic analysis of transient thermal and static loads was completed for engine startup to shutdown. These results have been compared to the original 2D analyses and to standard ASME Boiler Code. Several areas of high stress concentration were identified which necessitated the introduction of an additional heat shield to buffer the thermal gradients during the engine transients.

\section{WBS 11400: PSR Section}

A design review was held with recuperator manufacturing in July. The core. air inlet and outlet ducts, gas inlet and outlet ducts, mounts, thermally balanced restraint system (TBRS), end beam and mount drawings were reviewed by manufacturing.

Final assembly drawings completed and are undergoing final check.

Factory assembly of a Solar prototype core is in progress. All air cells have been manufactured for that core, and core assembly is in progress.

Transient structural analyses during start up of the recuperator are being performed. Preliminary results have shown that the air out duct tee is excessively stressed. The high stresses are due to thermal gradients that occur during start up. Structural supports are being redesigned to alleviate the thermal stresses that develop.

Sample welds of the tee to core weld were fabricated and analyzed. The analysis revealed poor penetration due to the difference in thickness of components. Modification to the weld preparation of the tee and the resulting impact on the structural integrity is being analyzed.

TBRS stud stress analysis was performed to validate change in thread. Gas ducting deflection analysis was performed to verify the clearance of stiffener tube during operation.

\section{WBS 11500: Fuel System -Mercury 50}

Ultra lean premix (ULP) combustion system: The majority of the drawings for the ULP 
combustion system assembly and the annular combustion test rig were released during this last reporting period for the manufacture of combustion development hardware. The combustion liner hole patterns have still not been fully defined but liner drawings were released to the allow design and manufacture of the required tooling.

A decision was made to modify and use Centaur 40 fuel manifolds for the Mercury 50 engine. CFD and flow modeling work has been continuing to support definition of the injector plenum air deflector baffle. Other sheet metal parts which go into the injector plenum, i.e., the air flow baffle and end plate heat shield are still being worked.

The injector design was released, but certain key dimensions will not be fully defined until test results from the prototype injector are obtained.

Concerns relating to the ability of the electro-chemical milling supplier to meet target dates for the turbulated liner cylinders forced a decision to go with wire turbulators for the first engine build. The ECM liners will now be used for the 2 nd development liner.

The first prototype Mercury 50 ULP gas only fuel injector was assembled and underwent cold flow testing to measure its flow effective areas for comparison with the design intent. The injector also was tested in a cold air flow combustor rig to visually observe the flame from the main and pilot systems. Thus far all testing has met expectations, but the design will not be fully qualified until testing at representative inlet air temperatures and pressures is completed. Manufacturing of components to make a full engine set for the annular combustion rig tests has been ongoing during this reporting period.

\section{WBS 11600: Turbine Assembly}

Stage 1 Blade: Mercury Machine Tool Company is in the process of developing the airfoil wax die and the electrodes for the ceramic core die. They have completed $40 \%$ of the wax die and approximately $80 \%$ of the ceramic core electrodes during this reporting period. They have also completed $20 \%$ of the machining fixtures. A drawing/tooling design review was held in July. Attendees included Solar personnel from design, heat transfer and layout as well as representatives from Howmet and Mercury Machine Company. An electrode review was held in September.

A critical design review (CDR) was held in late August for the stage 1 blade and disk. A few minor issues were raised, but the design team sanctioned the blade design. The design team has been evaluating three different suppliers for the abrasive tip coating deemed necessary for optimal engine efficiency. Solar and Howmet are in process of evaluating a Pt-Al coating/CMSX-10 material combination. Solar and Howmet are also of consolidating the thermal cycles for the CMSX-10 blade.

Drawings for the stage 1 disk and rim seals have been released for tooling purposes, with the disk rough machining drawing released to order actual parts. Rough machining disks have been received in house. Blade damper drawings have also been issued.

Stage 2 Blade: The second stage turbine blade drawing was issued this reporting period for tooling purposes. Release of the turbine blade casting definition has allowed for investment casting tooling procurement. Details of the blade firtree attachment have been thoroughly analyzed for adverse 
tolerance conditions and the design definition incorporated into the blade configuration. Similarly, rough machined disk definition release has stimulated progress on the Udimet 720 intermediate grain size development program. Ladish Corporation has developed forging die and ultrasonic inspection shapes and completed preliminary cooling rate studies. Wyman-Gordon Corporation completed the first four pancake forgings for Udimet 720 and has developed ultrasonic and heat-treat shapes from the released disk design definition.

Detail design of the turbine shaft and center-shaft has progressed over the last reporting period. Seal stress calculations for the threaded labyrinth seal were completed using ANSYS finite element modeling. The shape was optimized to fit in the available space with due regard to thermal and centrifugal deflections matched with acceptable stress. Curvic coupling separation forces and required assembly interference for pressing the seal disk on the turbine shaft were calculated and found acceptable. Both shafts modeled using finite element techniques and acceptable stresses/deflections were demonstrated as a result. Appropriate spline dimensions and pilot dimensions were assessed and incorporated into the design.

Stage 1 Blade Cooling Design: The cooling design of the first stage turbine blade was completed during the last reporting period. Design options were identified in an effort to reduce risk and to be rainbow-tested. A $15.4 \mathrm{X}$ scaled leading edge model with the screw-shaped swirl cooling configuration was tested for heat transfer coefficients using a transient liquid crystal technique. The measured values matched within $5 \%$ of the predicted values. A $7 \mathrm{X}$ polyurethane model of the overall cooling core is being constructed that will be used for a flow visualization and pressure drop and heat transfer measurements.

The aft nozzle support was redesigned during this reporting period in order to minimize thermal distortion.

A finite element thermal model is being constructed to provide transient response data for turbine tip clearance predictions. This model will include a $3 \mathrm{D}$ centerframe along with $2 \mathrm{D}$ combustor, turbine and compressor sections. The complete engine thermal model is $50 \%$ defined, pending completion of nozzle support/centerframe interface redesign. The thermal FE model will make use of a recently completed engine dynamic simulation database which predicts dynamic transient behavior characteristics for the recuperated Mercury 50 engine for all operating conditions.

\section{WBS 11800: Exhaust Assembly/Center Frame}

Center Frame: A design review of the center frame design was held in July. The review confirmed the acceptability the center frame under steady-state, and full load conditions. A simplified 3D finite element transient thermal and stress model for the center frame (based solely on the axial deflections) was completed in August.

Drawings for the forward and aft end plates have been issued for tooling purposes and have been sent to the supplier for procurement. The full center frame assembly supplier has been selected and is awaiting the full assembly drawing release.

The preliminary relief valve piping system were defined. Engine design is working closely with Packaging to assure that the system performs as required. 
Exhaust Collector: A Critical Design Review for the exhaust collector was held in July. Agilis representatives presented the current design and analysis to the Mercury design team. The design was approved, pending action items documented in the review. During this visit, Agilis and Solar's engineers visited Solar's collector supplier, Ketema. A few modifications to the design were incorporated based on this visit.

Drawings have been released to the supplier for tool fabrication. These drawings include the flange forging, the flange machining and the outer flowpath.

A stress analysis of the collector without heat shields resulted in a design modification to exclude the heat shield. This will reduce cost and the complexity of the assembly.

A CFD analysis was undertaken during this reporting period which demonstrated an increase in pressure recovery with the addition of a simple baffle plate. As a result of this analysis, a baffle plate will be added during engine development testing to verify predicted efficiency increases. If the test is successful, the plate will be introduced into the collector design.

A detailed conceptual study was undertaken by Agilis during this reporting period to investigate the feasibility of incorporating a hot strut design in place of the current EGV and nozzle case support system. The hot strut would mitigate the sensitivity of the stage 1 blade tip clearance from centerframe thermal effects. The conceptual design has been completed. A preliminary hot strut drawing will be prepared in order to send to potential suppliers for cost assessment.

\section{WBS 12110: Generator}

The generator specification was issued and Request for Quotes were issued to several suppliers. Only one supplier had responded as of the end of the quarter,.

\section{WBS 12140: Package/Generator Frame}

The basic skid was designed, and a CAD model was completed. The base design includes the generator support, oil tank (inclusive to frame/not removable), pivoting trunnion mount, 'soft foot' mount, tie down pads and lifting devices.

The recuperator support is still in the concept stage - no detail design was done this quarter.

\section{WBS 12150: Enclosure Assembly and WBS 12160: Ancillary Equipment}

Package heat rejection for ventilation requirements and package noise signature were established after technical interfaces between package and engine design engineers. Ancillary engineering finalized the air inlet duct configuration with a comprehensive interface with engine design engineering. The 'relief valve system' silencer design was modified to allow for updated design conditions. The associated control scheme was revised. The air inlet filtration system and the ventilation system for the enclosure were initially sized, thus allowing an assessment of the feasibility of innovative package design concepts. The 'drop-over' enclosure concept was presented at the package design review. Work was initiated on the design of an overhead engine 
removal system that will be integral with the package support structure.

\section{WBS 122210: Control Console}

Conceptual control concepts were developed and sketches reviewed sketches within the Controls Group. An input/outlet list was completed based on available current information relating to the engine and package equipment. A CO analyzer was selected on an interim basis.

\section{WBS 12220: Lube Oil System}

The lube oil system was $80 \%$ complete. Work was done towards selection of suppliers of oil pump/motor/valve assembly. A further iteration of the on-going update of lube oil schematic was completed. The thermo control valve was defined.

\section{WBS 12230: Fuel System}

Another iteration of the gas fuel schematic was completed. The design of the system is approximately $80 \%$ complete.

\section{WBS 12240: Start System}

Design of the start system is complete.

\section{2}

\section{TECHNOLOGY DEVELOPMENT PROGRAMS}

\section{WBS 19100: Advanced Turbine Cooling and Sealing}

Blade Screw Cooling: A test rig to evaluate the heat transfer coefficients in a screw-shaped vortex cooling chamber was completed at the University of Utah. The engineering report on the flow phenomena for this cooling technique was submitted to Solar. A test report on the effects of rotation on screw-shaped cooling effectiveness was completed at MIT and submitted to Solar.

Nozzle Film Cooling: Validation testing on the upscaled turbine nozzle film cooling test rig was completed. Testing included steady state and transient testing, as well as the secondary (coolant entry) flow systems.

Disk Cooling: The gas ingress test on the unsealed disk cavity was completed on the rig as a baseline for evaluating optimized turbine disk sealing and cooling. Comparison tests on the sealed disk cavity have been started.

Semi-active Tip Clearance Control: A Saturn engine demonstration test with semi-active tip clearance control was successfully completed, and results are under evaluation.

Other: A feasibility study was performed for application of dimples for heat transfer augmentation without and increase in pressure loss penalties. Results are promising for both 
turbine hot section and combustor liner cooling.

\section{WBS 19200: Advanced Combustion}

Ultra-lean Premixed (ULP)Combustor Development: CFD runs on the initial design of the Mercury 50 lean premixed injector indicate flow detaches from the downstream portion of the centerbody due to the flow expanding into the liner. This can be prevented by recessing the centerbody.

The first Mercury 50 prototype fuel injector was assembled and cold flow tested. A single injector test section was initially commissioned with a standard SoLoNOx injector. Testing of the Mercury 50 injector will begin next period.

Advanced Combustor Controls: Attempts to operate the CEMCAT carbon monoxide (CO) sensor on a wet (undried) gas sample failed. The need to dry the gas sample and control flow rate over the sensor requires nearly as much flow conditioning as a conventional CEMS system. On the other hand, testing at Servomex has shown that its $\mathrm{CO}$ sensor has the sensitivity necessary to measure concentrations of less than $50 \mathrm{ppm}$, without the complexity of the CEMCAT system.

Advanced Liner Cooling: Dimpled plates were prepared and tested in the heat transfer rig. This surface has demonstrated the required high heat transfer coefficients with very low pressure losses. This offers potential for significant improvement in the combustor cooling approach, and manufacturing methods are being evaluated.

Catalytic Combustion: Atmospheric rig tests of a subscale catalyst bed was completed at PCI. Local overheating of the catalyst was evident, and a redesign is in progress. At Catalytica, the subscale tests for durability and the effects of contamination continued. At Solar, the layout of the Mercury 50 combustion system was completed, and a very successful preliminary design review was held.

\section{WBS 19300: Ceramic and Composite Materials}

Ceramic Blade Development: The rub rig is being prepared to test ceramic blades (from CSGT) rubbing against an abradable tip shoe. Effort on the design of a ceramic blade for the Mercury has been delayed indefinitely due to funding limitations.

Ceramic Nozzle Development: A patent application for the long term conditioning of silicon nitride was finalized. No further effort will be spent on the ceramic nozzle at this time.

Ceramic (Composite) Interstage Seal Ring: The rub test disk knife edges were coated with chrome carbide, and the disk and CFCC seal ring are ready for rub testing.

\section{WBS 19400: Advanced Turbine Materials Development}

Forged Disk Development: Results of initial creep and rupture tests of course-grained Udimet 
720 at Ladish Company meet the minimum life targets established for the Mercury 50 second stage disk design. At the design operating temperature, the Udimet 720 disk is expected to have 20 times the life of a Waspaloy disk. Notch ductile behavior has been observed on all creep rupture specimens to date. Promising results were also obtained from the first low cycle fatigue tests at $1325^{\circ} \mathrm{F}$.

At Wyman Gordon, mechanical tests of the course grain material are $60 \%$ completed. Tensile tests at $1000^{\circ} \mathrm{F}$ and $1200^{\circ} \mathrm{F}$ exceed program targets and creep rupture tests are in progress. On this material notch brittle behavior was observed, which is a critical issue being investigated further. Pancake forgings for the first two Mercury 50 engines have been forged, heat treated, and ultrasonically inspected. Machining at the supplier will be completed next period, following release of the drawing.

Dual Property Disk: Hub materials and rim castings have been received, and samples are being tested for material properties. No further effort will be spent on this program at this time due to funding limitations.

Advanced Casting Techniques: The vacuum die cast mold for test bars was modified to provide straight test bars, and bars were received for materials property testing. A mold for the compressor blades was fabricated. Test blades will be cast during the next reporting period.

A sliding ring for the Mercury 50 engine has been spravcast and HIPed, reducing manufacturing cost. Test data shows properties similar to wrought ring-rolled Waspaloy.

Low Coefficient of Expansion Material: The first Mercury 50 nozzle support rings produced in Haynes 242 from 12" diameter billet were delivered. They are being used for mechanical testing and machining trials, and one ring will be made available for engine testing. Mechanical properties have met the specified requirements. The prime path material for this part is Waspaloy due to its superior high temperature properties.

Single Crystal Blade Material -CMSX-10: Experiments have led to a $33 \%$ reduction of solution heat treating cycle time, which is a cost saving factor. Mechanical tests are being made to determine the effects of the reduced solution time.

\section{WBS 19500: Recuperator Material Development}

Initial testing of the modified 347 SS has shown excellent results in both creep and oxidation tests. The modification is achieved via new thermomechanical processing techniques, and the material has an estimated creep life of two to four times that of standard 347 SS. A patent disclosure on the process has been initiated. This material is expected to increase recuperator life at $1200^{\circ} \mathrm{F}$ from 30,000 hours to 100,000 hours.

Other materials were reviewed as candidates to increase operating temperature capability to $1300^{\circ} \mathrm{F}$. Three candidates are being tested, and one shows good potential based on early creep tests, and another may actually be capable of $1400^{\circ} \mathrm{F}$ for low pressure applications.

\section{WBS 19700: Advanced Coating Systems}


Thermal Barrier Coatings: An instrumented test on a thermal barrier coated (TBC) combustor liner demonstrated metal temperature reductions up to $150^{\circ} \mathrm{F}$. A longer term test is being prepared. Engineering Design Memos (EDM) were issued on material property data measurements of plasma sprayed TBCs and on a life prediction method for plasma sprayed TBCs.

Thermal cycling tests to $2000^{\circ} \mathrm{F}$ were completed on four different $\mathrm{TBC}$ systems. The tests showed that TBC life is shorter for 10 -hour thermal cycles than it is for 100 -hour cycles. EB-PVD TBCs were applied on Taurus turbine blades for evaluation.

Rub rig tests of un-tipped blades against functionally graded material and thick ceramic abradable tipseals were completed at temperatures of $1100^{\circ} \mathrm{F}, 1650^{\circ} \mathrm{F}$ and $1800^{\circ} \mathrm{F}$. Some blade tip loss was observed, and evaluation is ongoing.

Internal Coatings: 1000 hours of oxidation tests at $1500^{\circ} \mathrm{F}$ and hot corrosion tests at $1200^{\circ} \mathrm{F}$ were completed on thin aluminide coatings on single crystal alloys CMSX-4. CMSX-10, and CMSX-11. In the hot corrosion tests, the coated samples had one tenth the weight change of the uncoated samples, indicating that the aluminide coating provides ten times the stability of the base alloy. They may help provide a component life of 5 to 10 times that of one of uncoated alloy. Chemical Vapor Deposition and Above the Pack processes were performed on sample blades. Uniform coating distribution and clean coating/substrate interface were accomplished. A successful cleaning process for internal coatings was established.

Alloy Coatings: The evaluation of as-coated Chromalloy UK Platinum-Aluminide on CMSX-10 single crystal alloy was completed. Results showed no topologically close packed (TCP) phase formation on the machined surfaces. In comparison, state-of-the-art coatings such as MDC-150 exhibited up to $1 \mathrm{mil}$ in TCP phase formation. After oxidation at $2100^{\circ} \mathrm{F}$ for 100 hours, the CUK coating exhibited 2.5 to 3 mils in TCP phases, whereas the MDC-150 coating exhibited 5-6 mils TCP phases. Oxidation testing at lower temperature is in progress.

\section{WBS 19800: Advanced Diffusers}

Diffuser tests with vortex generators installed demonstrated greater improvement on diffusers without collectors than those with collectors. Preparations are now in work for the half scale diffuser for the turbine test rig.

\section{WBS 19900: Man-Machine Interface}

All of the run-time services for the MMI have been demonstrated, as has the multi-language and historical data playback capability. The graphical editor toolkit has been integrated. Testing is continuing on the Integrated Development Environment and the View Editor. Development is also continuing on the integration of Visual Basic for Application to perform the math calculations and on the MMI setup programs for alarms and data.

\subsection{ATS L-ENGINE}




\section{WBS 202000: Engine Definition}

The ATS-L engine concept design was reviewed at a Concept Review meeting on July 10. All aspects of the engine and some of the package features were presented by the design engineering staff responsible for the various sections and/or overall engine configuration. Following this major review the efforts of the Design Team are focused on the preliminary design. The Preliminary Design Review is planned before the of 1997.

The engine design must meet the criteria specified in the ATS-L Business Plan and the Product Requirement Specification (PRS) incorporate in the plan. The document specifying the "L" engine design criteria were competed in early August. This document is a supplement to the PRS and provides specific technical criteria relating to the design of the engine.

Accessability will be a major factor in the "L" package design. Specific operations carried out during a standard overhaul of Solar's current products will be conducted in the field. Thus, the engine and package design will maximize the ability to change out components and parts in the field. Space requirements are also critical for the "L" package. Package footprint, volume, and height are to be kept as small as practical.

Preliminary design work has proceeded including a preliminary selection of materials for most components. A major focus of the effort was on the examination of alternative combustion schemes. Considerations include the ability to operate under part load conditions without performance penalties, and the ability to retrofit an ultra-lean premix combustion system to a catalytic design, rotor dynamics issues and product cost.

A detailed product cost analysis was undertaken using a bottoms-up approach involving a careful estimate of cost for the individual components and assemblies. The initial estimate indicates that the $L$ will be able to achieve the $10 \%$ cost of power reduction goal as well as more aggressive internal cost goals. The cost estimate will change further as the design becomes more detailed.

The "L" Development Plan is being refined along with the conceptual and preliminary design efforts. The basis for the plan are schedule, effort and cost estimates from the current ATS-L time line and Management Plan and data from the Solar ATS-L Business Plan. The estimates are being updated with bottoms-up cost estimates for the components and assemblies.

The schedule, effort, and cost estimates will be incorporated in a project plan being loaded into the Open Plan software. This software is currently the subject of a conference room pilot project at Solar; use of the software may ultimately be extended to encompass all of Solar's development projects. The plan enables rapid estimates of effort and cost and "what if" scenarios. A new program plan for the ATS-L should be in place before the end of November.

\subsection{TEST RIGS}

\section{WBS 42100: Compressor Rig Testing}

The Advanced Component Efficiency (ACE) compressor rig testing was completed at the end of July. The Phase $\mathrm{V}$ testing completed the optimum mapping with various vane positions. Exit 
traverse surveys determined that exit flow profiles per the NASA code over-predict blade row interactions. The code will be refined accordingly. Inlet duct straightener additions demonstrated no measurable differences compared to the original inlet configuration. A test summary report draft has been completed by Wright Patterson AFB with the final report expected by November.

The rig and test compressor were disassembled, and high cycle fatigue (HCF) cracking was found on the compressor stators of stages 5,6 and 7. An investigation was undertaken to find out the cause of the deficiencies, and the extent to which the current Mercury 50 stator design would need to be modified to ensure hardware longevity. Following an analysis of the modal characteristics of the stators, the team and concluded that stator 4 needed minor redesign to avoid a sixth engine order interference. This redesign has been successfully accomplished without negative impact to schedule or to downstream aerodynamics.

In addition to the stator cracking, the ACE rig exhibited stage 9 disk cracking. Investigation into the possible causes was ongoing at the close of the reporting period.

\section{WBS 42210: Ultra Lean Premixed (ULP) Combustor Test Rig}

The combustor housing end cover has been cast and delivered to Solar for machining. The combustor housing itself is currently being cast. The first prototype fuel injector has been assembled. The test facility is being modified to accept the combustor rig, and testing is scheduled to begin in December.

\section{WBS 42400: Turbine Test Rig}

Airfoil designs were completed, and procurement of test hardware is in process. First stage nozzle flow tests were completed, and the flow capacity agrees with design calculations although the nozzle configuration used does not account for the effect of cooling flow on the overall turbine. Baseline testing with the full two-stage rig is scheduled to start in December.

\section{WBS 42500: Squeeze Film Damper Test Rig}

A squeeze film damper from Waukesha has completed its characterization testing, and the Solar damper is being fabricated for comparison testing.

\section{WBS 65000: RAMD}

The specification for the RAMD Interface/Display System has been completed identifying inputs, data processing requirements, workstation and presentation program requirements, system operation and maintenance manuals and other documentation.

\subsection{COMMERCIALIZATION}

WBS 52500: Product Cost Management 
The current estimate of the engine cost is comfortably below the program goal of $10 \%$ reduced cost of power although the estimated product cost increased slightly during the past quarter as current quoting is showing higher than expected costs on some components. Of the drawings released, $32 \%$ have current quotes. Costs under scrutiny and receiving significant attention include the Stagel nozzle, center frame, combustor end cover, compressor rotor spacers, air divertor valve, and package controls. In all cases, cross-functional groups are looking at cost reduction options to get cost and target to align.

\section{WBS 62000: Manufacturing Readiness Plan}

Work continues on the Readiness Plan in the Cold CAM, Hot CAM, Rotor CAM and Controls. All areas are at different phases of the project, and work is progressing smoothly. Recently, a new shop layout was selected by the Cold CAM. This layout includes a significant amount of floor space for a stand-alone Mercury Cell. This cell will manufacture many parts including the air inlet, diffuser, compressor case, and gear box housing and will also have an assembly area. The cell will be designed so that at some point it could be relocated as a part of a Mercury specific factory, if required.

The Rotor CAM is evaluating long range strategies in concert with shop layouts. Significant work will have to take place to accommodate the equipment required to manufacture the hardware forecasted for five years out and beyond.

Hot CAM and Controls are in the load analysis and assessment stage. The bottlenecks and problem areas are not yet clearly defined. Studies are ongoing. At some point both areas will progress to the current stage of the Cold CAM.

\section{WBS 71100: Host Site Research and Selection}

Solar and Rochelle Municipal Utilities (RMU) moved closer to locating a host site for the 8,000 hour demonstration of the Mercury 50. Solar's ATS Commercialization Manager made a presentation in late July with RMU and members of local industry in Rochelle, IL regarding the ATS program, Solar's ATS product, and the selection of the host site. Following continued strong interest. an August meeting was held in Rochelle with the GM of RMU and representatives of Hormel Foods. In September, RMU's General Manager and Solar's Commercialization Manager made a joint presentation to DOE's Steve Waslo. Pat Hoffman and Lisa Barnett, as well as to Solar's ATS and Sales \& Marketing personnel. They proposed that RMU locate the demonstration unit in its service area. The presentation was favorably received by all parties. A proposal was later sent to RMU. Communications are ongoing to finalize an arrangement.

\subsection{PROGRAM MANAGEMENT}

Program Activities: Considerable efforts were initiated in August, as part of the company's annual budget planning process, to reforecast and control ' 97 and '98 ATS labor and material expenditures. The continuing shortfall of DOE funding from levels identified in the cooperative 
agreement together with heavy demands on Solar resources for product development and support, as well as the constant need to meet investors' financial performance expectations, has forced Solar to commence selective reductions or delays in program activities. These reductions also are reflective of the progress made to date on engine design and analysis and technology development activities. As with any relatively high risk $R \& D$, parallel pathways were required to be pursued until sufficient knowledge and data was available to make design decisions and technology down selections. Solar has reached a stage where it may commence such selective reductions consistent with its RD\&T plan.

A detailed investigation and analysis of technology development and test rig planned activities identified potential cost savings as described furthre below. The ground rules were to identify those activities that are not deemed absolutely critical to (i) preserve Mercury 50 the schedule for the initial test units and (ii) meet ATS performance requirements.

Ceramic blade and nozzle design and development programs have been placed on hold. Efforts related to basic material characterization will continue at a minimal level, but the plan to test ceramic components in the field demonstration (and the development engine) has been canceled.

Continuous Fiber Ceramic Composite (CFCC) programs have similarly been reduced to basic material characterization. The CFCC transition duct will continue to be considered for a back-up for the $L$ engine.

An effort to establish a life prediction model for the TBC turbine blades has been canceled at the Southwest Research Institute. TBCs may be applied to blades on either the S or L engines, but the insulating benefit is not being considered in the design life due to the uncertainty in TBC adherence life.

Effort planned to establish a life prediction model for superalloy turbine disks has been eliminated from the ATS technology program.

Down selection to a single supplier for the Udimet 720 turbine disk material will occur at the beginning of 1998 rather than later in the year. At this time the material from one supplier is meeting all the material test goals, and the early down selection will add minimal risk to the success of the material development program.

Development of the dual alloy turbine disk will be discontinued, except for continuation of material creep testing already underway. The primary need for the dual alloy disk was for supporting the ceramic blades, which would typically operate with a higher disk rim temperature.

Development of alternate recuperator foil materials to extend the service life and operating temperature capability have been reduced. Initial test results of the $1200^{\circ} \mathrm{F}$ material are encouraging and indicate a substantial life increase over standard 347 stainless steel is possible. Efforts to develop a higher temperature material capable of $1300^{\circ} \mathrm{F}$ have been eliminated from the program.

ATS funding of the vacuum die casting and spray casting process development efforts for product cost reduction has been stopped. This activity will be continued by Howmet as a part of their internally-funded technology development activities. 
A portion of the combustion research testing not considered vital for the ATS program has been terminated. Similarly, portion of the tests planned for the half scale turbine test rig have been eliminated from the test program because they are not vital to support the initial development of the Mercury 50. Tests of the turbine diffuser and collector have also been reduced to the minimum considered necessary. A plan to test a scale model of the compressor inlet has been canceled because of high confidence in the analytical model, and the low risk of an adverse consequence on performance.

Although the above program revisions add some risk to the attainment of low cost and long life goals, they are considered low risk as far as engine schedule and performance are concerned. Some of the eliminated or reduced activities may resume later as deemed necessary for the achievement of program objectives.

ATS Program Status: The Financial Status Report (SF 269) and Federal Assistance management Summary Report (DOE Form F4600.5) for the month of September are attached.

As of the end of September there was an unfavorable $53.6 \%$ schedule variance against the 6 year baseline for the overall ATS program (59.5\% last quarter). This is attributable to (i) continuing funding constraints and (ii) a $0 / 100$ percent complete assumption for program milestones. Solar also shows an unfavorable cost variance of $15.1 \%$ (16.21\% last quarter) that is due in large part to the high degree of outsourcing. The unfavorable variances are expected to continue to diminish somewhat over the next few months as preliminary drawings are issued.

The Mercury 50 Team is holding to the key target of a March 1998 commencement of assembly on the first engine build. The principal items currently pacing the Mercury 50 schedule are:

- $\quad$ Stage 1 and 2 Turbine Blades

- $\quad$ Stage 1 Turbine Nozzle Segment

- $\quad$ Stage 1 and 2 Turbine Disks

- $\quad$ Stage 10 Compressor Aft Hub

- $\quad$ Stage 10 Compressor Outlet Guide Vane

- $\quad$ Stage 9 and 10 Compressor Blades

- $\quad$ Center Frame Structure

- Rotor Bearings

Based upon the components that are currently most critical to schedule, it is estimated that the Mercury program is some four months behind schedule. 
1 abed

L6/0ع/Ol :pә|u!!
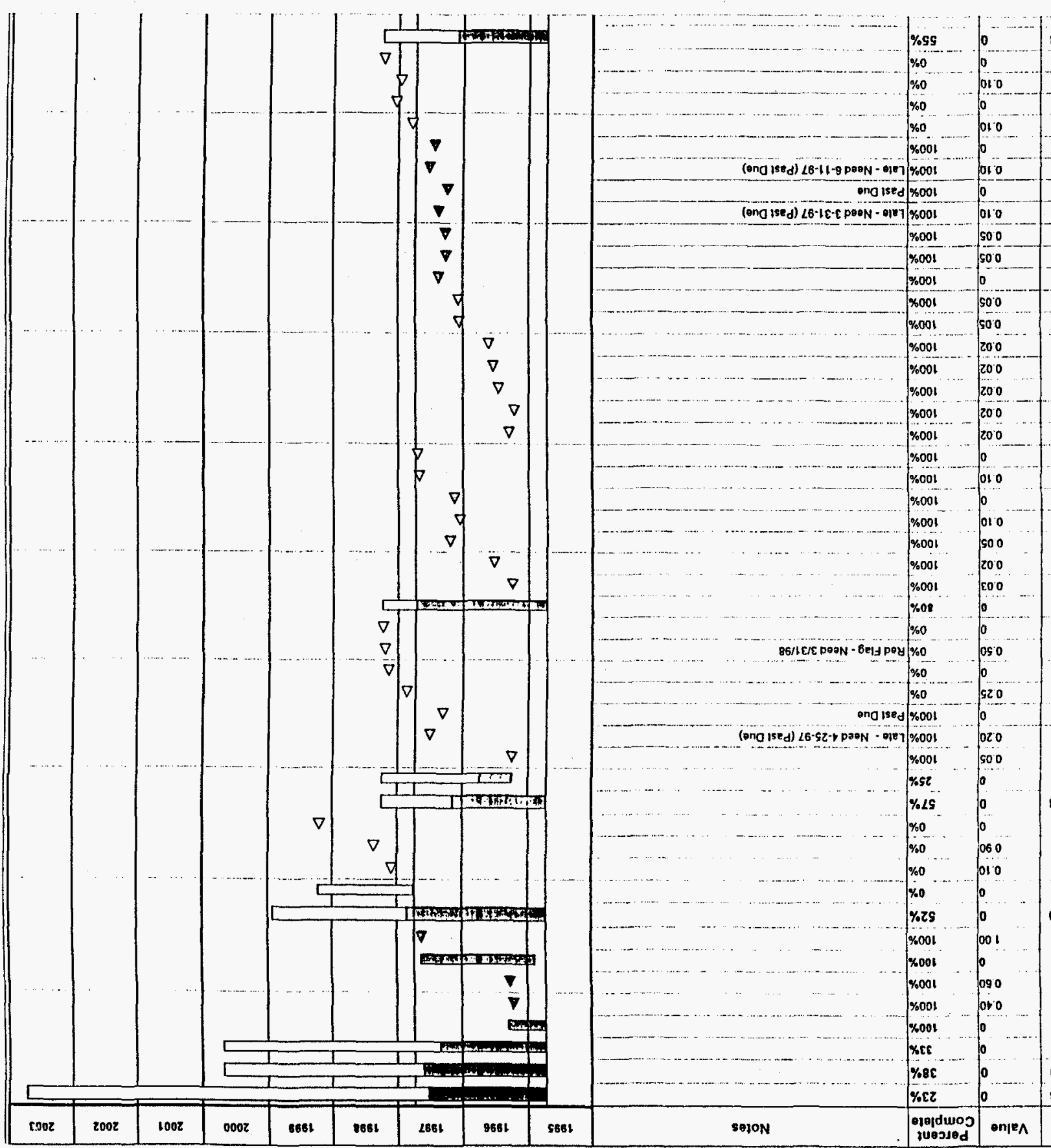

\begin{tabular}{|c|c|c|c|c|}
\hline lete & $56 / 71 / 6$ & snopuen & S. Uoppos losseidwo? & 00241 \\
\hline $1 \varepsilon / \varepsilon$ & $86 / L / 6$ & $001 \cdot 0905 r z$ & 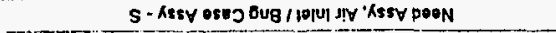 & szoz111 \\
\hline L6/2ZZZ: & $26 / 1+2 / 21$ & $001.0905 r z$ & 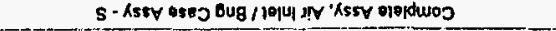 & nzozi11 \\
\hline $86 / \mathrm{l} / 4$ & $86 / \mathrm{ini}$ & 090stz [ & 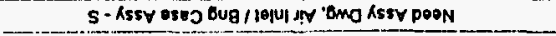 & $\varepsilon 20211 !$ \\
\hline $26 / 91 / 0 !$ & $26 / 5101$ & ogossz & 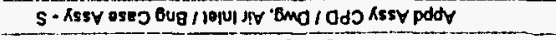 & 22024 \\
\hline L8/49 & $26 / 11 / 9$ & reosst & s - oses Buyzeg in Ang Bunumpew poon & 120211 \\
\hline $26 / 8 / 2$ & $26 / 8 / 4$ & rossrz & 5 - eseo Buyesg in 'Ano / ado Bunumbew pddy & oz $021+1$ \\
\hline$\ell 6 / 1 E / E$ & $\angle 6 / \mathrm{LER}$ & s90stz & S - oseo buyeog in 'Gma Bunseo peon & 8102111 \\
\hline LB/6//s & $26 / 61 / 9$ & s905rz & 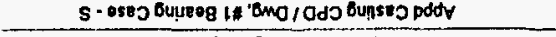 & 8102111 \\
\hline $26 / 8 / 1$ & $26 / 6 / t$ & 1-2905rz & s. Assy Bureeg Joploy 'Od contd & 210241! \\
\hline LG/SIT & $26 / 910$ & $\angle 90 s+2$ & s- Kstry Buveog Jelloy ismo/ ado pddy & 9102111 \\
\hline $26 / 02 / 5$ & $26 / 02 / 5$ & & S- (papeon H) Ueld Buyeog dnyjeg WeIS & stozIII \\
\hline 260001 & L6/081! & & 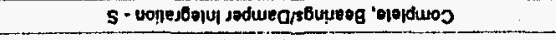 & rozil1 \\
\hline$\angle 6 i+2 \pi$ & $\leq 6 / 2 \pi \mid$ & & 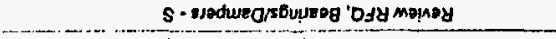 & E102111 \\
\hline $96 / 8 / 9$ & $96 / 8 / 8$ & & 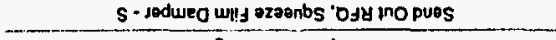 & 2102111 \\
\hline $95 / 21 / 2$ & $96 / 4 / 4$ & & S- 8604898 soloy ojy no purs & "102111 \\
\hline $96 / 1 / 19$ & $96 / 1 / 19$ & & 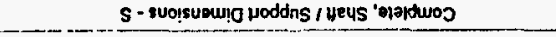 & 0102111 \\
\hline $96 / \mathrm{k} / \mathrm{c}$ & 96/the & & 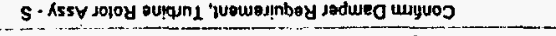 & $60211 !$ \\
\hline $96 / 91 / \%$ & $96 / 91 / 4$ & 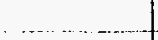 & 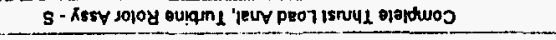 & 802111 \\
\hline L6/9 V6 & $26 / 51 / 6$ & $190 s+2$ & 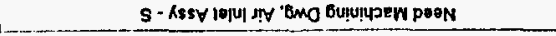 & L202111 \\
\hline $28: 26$ & L6/2/6 & $19054 z$ & 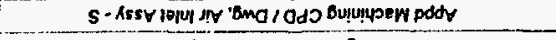 & $9024 !$ \\
\hline $26112 \pi$ & LE/2ZI] & z90s?z & 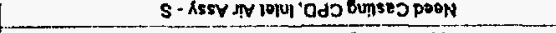 & sozil \\
\hline 16/zz!! & 26/22]! & $290 s s z$ & 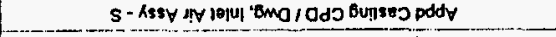 & r.ozu! \\
\hline$\angle 641 / \varepsilon$ & L6/LU/2 & $001-0905 \vee 2$ & 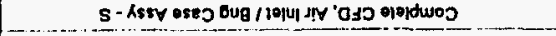 & coz!! \\
\hline $96 / 01 / 4$ & $06 / 01 / 4$ & $001-09092 z$ & 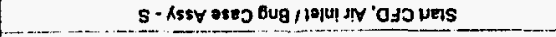 & zoz!! \\
\hline 96///7 & $96 / / 4$ & .001-090svz & 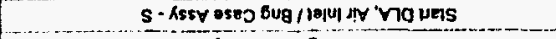 & cor!! \\
\hline $86611 / \varepsilon$ & S611/6) & - lo1-090sez & 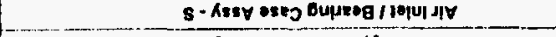 & 0211 \\
\hline 86/LE/E & $86 / 1 / \mathrm{C}$ & $001-5 \cos 2 z$ & 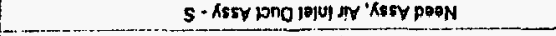 & Lo11! \\
\hline $86 / 61 / \varepsilon$ & $86 / 61 / \kappa$ & $001-5 s 05+2$ & 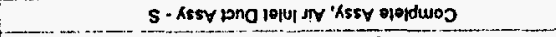 & 90111 \\
\hline B6//ع & 86/4C & scosiz & 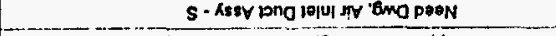 & 011 \\
\hline$\angle 6 / S I / I !$ & $26 / 51 / 1 !$ & sgostz & 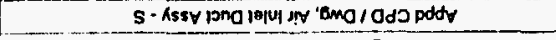 & roll1 \\
\hline $26 / 52 / 4$ & $\leq 6 / 524$, & 9sospz. & 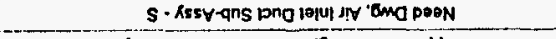 & Eo14! \\
\hline $26 / 2 / 2$ & $26 / 2 / 4$ & 9s0siz & 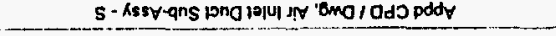 & 201311 \\
\hline $96 / 1 / 4$ & $96 / / \pi$ & $001-5505+2$ & 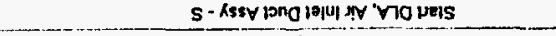 & 104111 \\
\hline $86 / 2 E / c$ & $96 n !$ & $001-5 s 058 z$ & 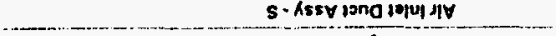 & t4! \\
\hline $86 / L E / E$ & $56 / 71 / 6$ & snoupa & 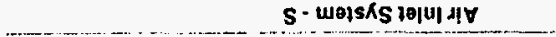 & 00111 \\
\hline $66 / 81 / 2$ & 66/81E & & 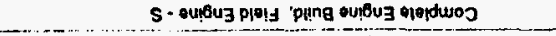 & E.01011 \\
\hline 86/si/s & 86/51/5 & & 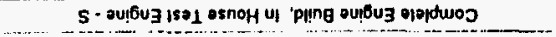 & 201011 \\
\hline $86 / v / t$ & $86 / \mathrm{r} /$ & & 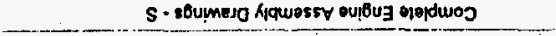 & 01011 \\
\hline 66/91/2 & $26 / 6 / 0)$ & & s- pyng outguz & 01041 \\
\hline $66 / / 2 \pi$ & $96 / 71 / 6$ & & $S$ - Alquess $\forall$ oupbug & 00011 \\
\hline 26/51/8 & L6/S1/8 & & 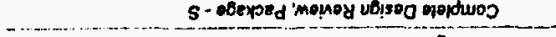 & 100801 \\
\hline 26/SLI/ & 581!1!!! & & s- Honluyiog obexord & oocos \\
\hline 96/CA & $96 / \mathrm{c} / \mathrm{t}$ & & $s$ - (zOd) monoy uelsoa Neunumpsd & 200204 \\
\hline $96 / 19 / / 2$ & $96 / 91 / \varsigma$ & & 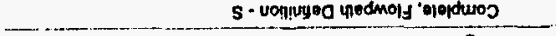 & 100201 \\
\hline $10 \mathrm{kn}$ & S6/1/6 & & s- & $\infty$ \\
\hline OO/LE/ & S6194/6. & & 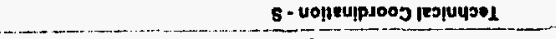 & 00101 \\
\hline $00 / 18 / 8$ & S6/R2/6 & & 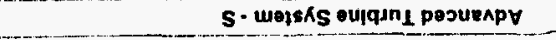 & 00001 \\
\hline $80 / 81 / 6$ & $S 6 / P L / 6$ & & 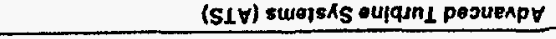 & $2 \angle 09$ \\
\hline $\begin{array}{l}\text { eleo } \\
\text { pug }\end{array}$ & $\begin{array}{l}\text { ejea } \\
\text { jeis }\end{array}$ & on $\mu \mathbb{R}_{\mathrm{d} / \mathrm{Bma}}$ & Ins & sem \\
\hline
\end{tabular}

mej601d |e|OL : Je|l!

t pue $\varepsilon$ әseud

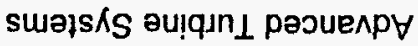


Advanced Turbine Systems

Phase 3 and 4

Filter: Total Program

\begin{tabular}{|c|c|c|c|c|}
\hline WBS & Task Name & Dwg / Part No & $\begin{array}{l}\text { Start } \\
\text { Date }\end{array}$ & $\begin{array}{l}\text { End } \\
\text { Dato }\end{array}$ \\
\hline 11210 & Comprassor Rotor Assembly - 3 & $245070 \cdot 100$ & 211498 & $3 / 31 / 88$ \\
\hline 11210.1 & Slart DLA, Compr Rolor Assy - $s$ & $245070-100$ & $4 / 1 / 96$ & 41/1/96 \\
\hline 11210.2 & Start CFD, Compr Rotor Assy - $S$ & $245070-100$ & $4 / 1196$ & $41 / 196$ \\
\hline 11210.3 & Appo Forging CPD / Owg, Fwd Compr Cone - $S$ & 245076 & 5/1997 & $5 / 19 / 97$ \\
\hline 11210.4 & Need Forging Dug, Fud Compr Cone - $\mathrm{s}$ & 245076 & $415 / 97$ & $4 / 5197$ \\
\hline 11210.5 & Appd CPD I D o Fud Compr Cono-s & 245075 & |ion17i97 & io/17/97 \\
\hline 11210.6 & Need Owg, Fwd Compr Cone - 5 & 245075 & $5 / 18 / 97$ & $5 / 18 / 97$ \\
\hline 112107 & Compleale Aero Dosign, Compr Blado Stg $1-\mathrm{s}$ & $245090-1$ & $9120 / 96$ & $8 / 20 / 96$ \\
\hline 11210.8 & Complele Mech Design, Compr Blade Slg 1.5 & $245090-1$ & $9 / 27796$ & $9 / 27 / 96$ \\
\hline 11210.9 & Appod CPD / Dugg, Compr Blade Stg 1.5 & 245090 & $5 / 21 / 97$ & $5 / 21 / 97$ \\
\hline 11210.10 & Need Dug, Compr Blado Sig 1-s & 245090 & 78897 & 718197 \\
\hline 11210.11 & Appd Forging CPD I Dwg, Compr Disk Stg i-s & 245089 & $10 / 31997$ & 10/3/97 \\
\hline 11210.12 & Noed Forging Dwg, Compr Disk Sig i.s & 245089 & 6011097 & $6 / 10997$ \\
\hline 1121013 & Appd Machine CPD I Dup Compr Disk Stg 1. S & 245110 & $8 / 26,97$ & $9 / 26 / 97$ \\
\hline 11210.14 & Need Machine Dwg Compr Disk Stg i - 5 & 245110 & $6 / 26 / 97$ & $6 / 26 / 97$ \\
\hline 11210.15 & Appd CPD / Release Dwg, Compr Disk Assy Slg 1-S & 245110 & $9 / 2669$ & $9 / 26 / 97$. \\
\hline 11210.16 & Need Dug. Compr Disk Assy Sig 1 - 5 & 245110 & $9 / 15197$ & $9 / 15197$ \\
\hline 11210.17 & Appd CPD, Now Cunvic Tooth Owg & 105242 & 421197 & 421197 \\
\hline 11210.18 & Neod Dug. Now Cunvic Tooth Dwg & 105242 & $3 / 3 / 97$ & 3/3/97 \\
\hline 11210.19 & Complete Aero Design, Compr Blade Sig 2 - $\mathrm{s}$ & $245100 \cdot 9$ & $9 / 20196$ & $9 / 20196$ \\
\hline 11210.20 & Completo Mech Dosign, Compr Blado Sig 2.S & $245100-1$ & 924196 & 92496 \\
\hline 11210.21 & Appd CPO / Owg. Comer Blade Slg $2 . \mathrm{s}$ & 245100 & $5 / 11197$ & $51 / 197$ \\
\hline 11210.22 & Need Dwg, Compr Blade S1g 2-5 & $245100^{\circ}$ & $778 / 97$ & $7 / 8997$ \\
\hline 11210.23 & Appd Forging CPD / Owg Compr Disk Sig 2.S & 245097 & 730097 & $7 / 30 / 97$ \\
\hline 11210.24 & Noed Forging Dwg. Compr Disk Sig 2 - & 245097 & 6,10197 & $6 / 10 / 97$ \\
\hline 11210.25 & Appd Machino CPD/ Dwg Compr Disk Stg 2 -S & 245120 & $915 / 97$ & $9 / 5 / 97$ \\
\hline 11210.26 & Need Machine Dug. Compr Disk Stg 2-S & 245120 & $6 / 6697$ & 6 \\
\hline 11210.27 & Appd CPD / Dwg, Compr Disk Assy Silg 2.s & 245120 & $9 / 5 / 97$ & $91 / 197$ \\
\hline 11210.28 & Need Dug, Compr Disk Assy Sig 2.5 & 245120 & $9 / 15 / 97$ & $8 / 15 / 97$ \\
\hline 11210.29 & Complete Aero Design, Compr Btado Sig $3 \cdot \mathrm{s}$ & $245115-1$ & $9 / 26 / 96$ & $9 / 26 / 96$ \\
\hline 11210.30 & Complete Mech Design, Compr Blade Sig 3 . $\mathrm{S}$ & $245115-1$ & $12 / 16 / 96$ & $12 / 16 / 96$ \\
\hline 11210.302 & Appd Casting CPD / Oung Compr Blade Stg 3 - S & 245116 & 411199 & 411197 \\
\hline $11210.30 \mathrm{~b}$ & Noed Casting Owg, Compt Blade Sig 3 - S & 245116 & $4123 / 97$ & $4 / 23 / 97$ \\
\hline 11210.31 & Appo CPD I Dugg, Compr Blade Stg 3 - S & 245115 & 9/241997 & s/24/97 \\
\hline 11210.32 & Need Owg. Compr Blade $\mathrm{Sig}_{3}$ - S & 245115 & 77897 & $7 / 8 / 97$ \\
\hline 11210.33 & Appos Casting CPo I Dug Compr Disk Sig $3 . \mathrm{s}$ & 245112 & $6 / 2197$ & $6 / 2 / 97$ \\
\hline 11210.34 & Noed Casting Dwg, Compr Disk Sig 3 - S & 245112 & $610 / 97$ & $6 / 1099$ \\
\hline 11210.35 & Appd Machine CPD / Dwg. Compr Disk Stg 3.5 & 245130 & $9 / 5197$ & 815197 \\
\hline 11210.36 & Need Machine Dug. Compr Disk Slg 3 .S & 245130 & $6 / 26197$ & $6 / 26 / 97$ \\
\hline 11210.37 & Releaso Dugg, Compr Disk Assy Sto 3.5 & 245130 & $9 / 5 / 97$ & $915 / 97$ \\
\hline 11210.38 & Need Dwg, Compr Disk Assy Sig 3.S & 245130 & g/15/97 & 2/15/97 \\
\hline 111210.39 & Complete Aero Desion, Comps Blade Sig 4- $\mathrm{S}$ & $245131-1$ & $10 \mathrm{or} 986$ & $1007 / 96$ \\
\hline 11210.40 & Complete Moch Design Compr Biade Slg 4 - $\mathrm{s}$ & $245+31-1$ & $10 / 10 / 96$ & 10/10/96 \\
\hline $11210.40 \mathrm{a}$ & 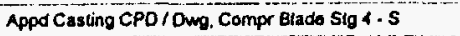 & $245+32$ & 429197 & $4 / 29 / 97$ \\
\hline $11210.40 \mathrm{~b}$ & Need Casting Dwg, Compr Blade Stg 4 - S & 245132 & $3 / 24 / 97$ & $3 / 2467$ \\
\hline 11210.41 & Appo CPD/OWog, Compr Blade Sig 4 - $s$ & 245131 & $9 / 25 / 97$ & 9/25/97 \\
\hline 11210.42 & Neod Dug, Compr Blade Sig 4 - 5 & 245131 & 781897 & 78899 \\
\hline 11210.43 & Appd forging CPD / Dwo. Compr Disk Sig 4. S & 24512 & $6 / 23 / 97$ & $6 / 23197$ \\
\hline 11210.44 & Neod Forging Dwg Compr Disk Sig $4 . \mathrm{s}$ & 245127 & 6/10/97 & $6 / 10 / 97$ \\
\hline
\end{tabular}

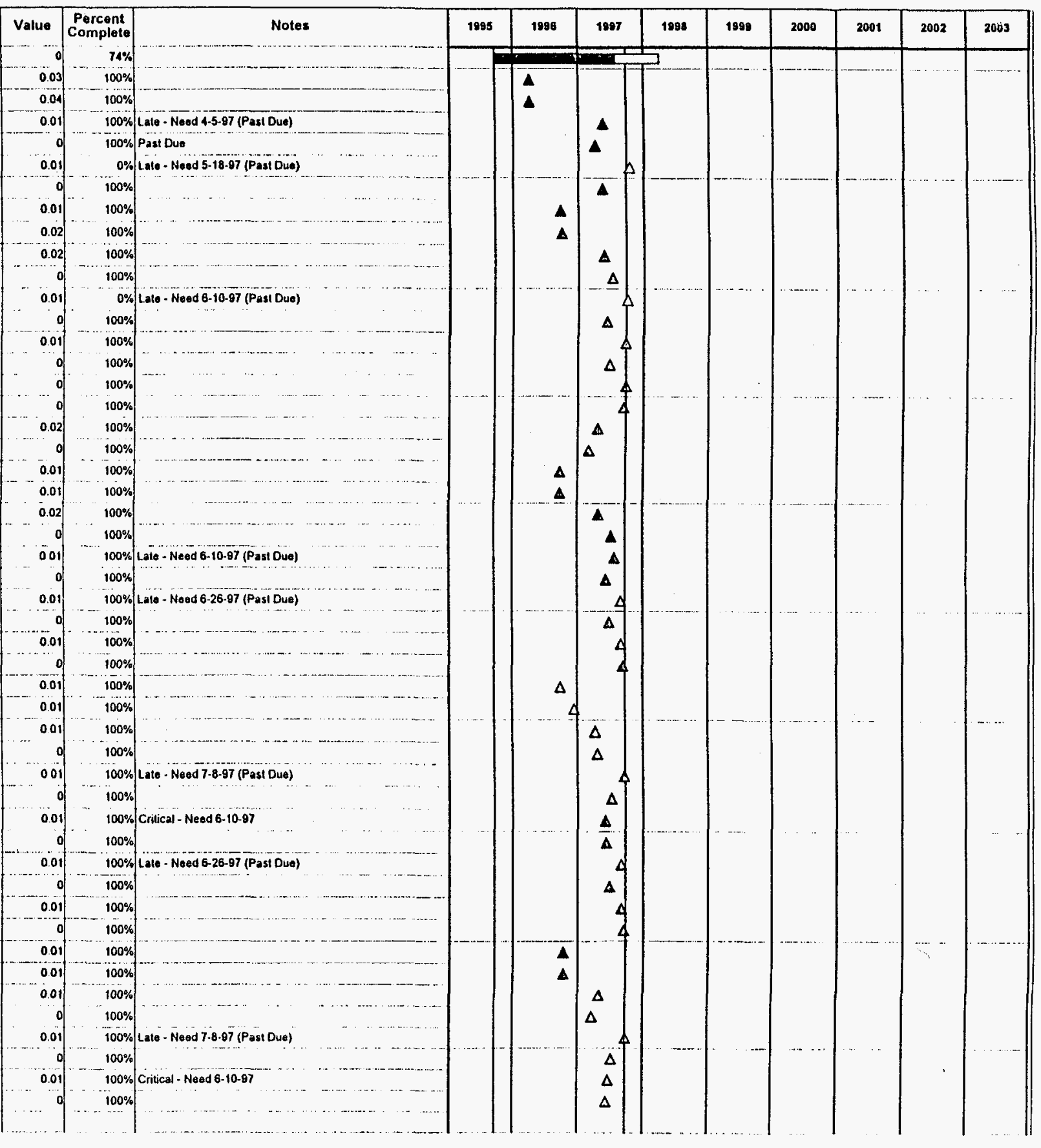

Printed: 10/30/97

Page 2 
Phase 3 and 4

Filter: Total Program

\begin{tabular}{|c|c|c|c|c|c|c|c|c|c|c|c|c|c|c|c|c|}
\hline WBS & Task Name & Dwg / Part No & $\begin{array}{l}\text { Start } \\
\text { Date }\end{array}$ & $\begin{array}{l}\text { End } \\
\text { Dato }\end{array}$ & Value $c$ & $\begin{array}{c}\text { Percent } \\
\text { Complete }\end{array}$ & Notes & 1995 & 1996 & 1997 & 1998 & 1989 & 2000 & 2001 & 2002 & 2063 \\
\hline$\$ 1210.45$ & Appod Machino CPO / Dugg, Compr Disk Sig 4 - $S$ & 245140 & $9 / 5 / 87$ & $9 / 5 / 97$ & 0.01 & $100 \%$ & Late Need 6.26.97 (Pasi Dua) & & & & & & & & & \\
\hline 11210.46 & Noed Machino Dug. Compr Disk Sig 4 - s & 245140 & $8 / 26 / 97$ & $6 / 26 / 97$ & & $100 \%$ & & & & $\Delta$ & & & & & & \\
\hline 11210.47 & Relea ase Dugg, Compr Disk Assy Sto 4-s & $245140^{-}$ & $8 / 5 / 97$ & ///5/97 & 0.01 & $100 \%$ & & & & & & & & & & \\
\hline 11210.48 & Need Owg, Compr Disk Assy Sig $4 . S$ & 245140 & $8 / 15187$ & $9 / 15 / 97$ & & $100 \%$ & & & & & & & & & & \\
\hline 11210.49 & Complete Aero Design, Compr Blado Slo 5 . S & $245145-1$ & $4 / 25 / 97$ & $4 / 25 / 97$ & 0.01 & $100 \%$ & & & & $\Delta$ & & & & & & \\
\hline 11210.50 & Complato Mech Design, Compor Blado Sig 5 - $s$ & $245145 \cdot 1$ & $4 / 25 / 97$ & $4 / 25 / 97$ & 0.01 & $100 \%$ & & & & $\Delta$ & & & & & & \\
\hline $11210.50 \mathrm{~s}$ & Appd Casting CPD / Owg, Compr Blado Sto 5 - 5 & 245146 & $4 / 15 / 97$ & $1 / 15 / 97$ & & $100 \%$ & & & & $\therefore$ & & & & & & \\
\hline $11210.50 \mathrm{~b}$ & Need Casting Dwg. Compr Blado Sto 5-S & 245146 & $3 / 2497$ & $3 / 24 / 97$ & & $100 \%$ & & & & $\boldsymbol{A}$ & & & & & & \\
\hline 11210.51 & Appd CPD / Owg, Compr Blade Sig 5.S & 245145 & $9 / 26 / 97$ & $9 / 26 / 97$ & 001. & $100 \%$ & Late - Need 7-8.97 (Past Dua) & & & & & & & & & \\
\hline 11210.52 & Noed Dug. Compr Blade Sig 5.S & 245145 & $788 / 97$ & $78 / 97$ & & $100 \%$ & $\ldots$ & & & $\Delta$ & & & & & & \\
\hline 11210.53 & Appd Forging CPO I Owg. Compr Disk Slb 5 - $S$ & 245142 & $5 / 15 / 97$ & $5 / 15 / 97$ & & $100 \%$ & & & & $\Delta$ & & & & & & \\
\hline $\begin{array}{l}11210.54 \\
11210.55\end{array}$ & $\begin{array}{l}\text { Noed Forging Durg. Compr Disk Slg } 5.5 \\
\text { Appd Machine CPD / Dug. Compr Disk Sig } 5 \text {. S }\end{array}$ & $\begin{array}{l}245142 \\
245150\end{array}$ & $\begin{array}{l}6 / 10 / 97 \\
9 / 5 / 97\end{array}$ & $\begin{array}{l}\text { 6/10/97 } \\
9 / 5 / 97\end{array}$ & & $\begin{array}{l}100 \% \\
100 \%\end{array}$ & Lale - Need 6-26-97 (Past Oue) & & & $\Delta$ & & & & & & \\
\hline 11210.56 & Need Machino Dwg, Compr Disk Sig $5 . \mathrm{S}$ & 245150 & $6 / 26 / 97$ & $6 / 26 / 97$ & & $100 \%$ & (n) & & & $\Delta$ & & & & & & \\
\hline 11210.57 & Appd CPD / Dwg Compr Disk Assy Stg 5 - $s$ & 245150 & $9 / 5 / 97$ & 9/5/97 & & $100 \%$ & & & & & & & & & & \\
\hline 11210.58 & Noed Dugg, Compr Disk Assy Sto 5-S & 245150 & $9 / 15 / 97$ & a/15/97 & & $100 \%$ & & & & & & & & & & \\
\hline 11210.59 & Complete Aero Design, Compr Blado Stg 6 - S & $245161-1$ & $5 / 1 / 97$ & $5 / 197$ & & $100 \%$ & & & & $\Delta$ & & & & & & \\
\hline 11210.60 & Complete Mech Design, Compr Btade SIg 6.S & $245161-1$ & $5 / 6 / 97$ & $5 / 6 / 97$ & 0.01 & $100 \%$ & & & & $\overline{\mathbf{\Delta}}$ & & & & & & \\
\hline 11210.602 & Appa Cas sing CPD 1 Owg, Compr Blade Stg 8-S & 245162 & 7722197 & 7222187 & 0.01 & 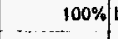 & Late - Need 4-23-97 (Past Due) & & & $\Delta$ & & & & & & \\
\hline 11210.606 & Need Casting Dugg Compr Blade Stg 6-S & 245162 & $1 / 23 / 97$ & 423397 & & $100 \%$ & Past Que & & & $\Delta$ & & & & & & \\
\hline 11210.61 & Appd CPD / Dugg, Compr Blado Stg 6.5 & 245161 & $10 / 10 / 97$ & 10/10/97 & 0.01 & $0 \% / 1$ & Lato - Need 7-8-97 (Past Due) & & & & & & & & & \\
\hline 11210.62 & Need Dwg, Compr Blade Stg 6.5 & 245161 & 77897 & $778 / 97$ & & $100 \%$ & $\ldots . .$. & & & $\Delta$ & & & & & & \\
\hline 11210.63 & Appo Forging CPD / Dupg, Compr Disk Sig 6 - $S$ & 245157 & $5 / 27 / 97$ & $5 / 27 / 97$ & 0.01 & $100 \%$ & . & & & 4 & & & & & & \\
\hline 11210.64 & Need Forging Dugg, Compr Disk Sig 6 - S & 245157 & 6/10/97 & $6 / 10 / 97$ & & $100 \%$ & $\ldots \ldots+\ldots$ & & & $\Delta$ & & & & & & \\
\hline 112065 & Appd Maching C CD / Dug Compr Disk Sig 6.S & 245160 & $9 / 5 / 97$ & $9 / 5 / 97$ & 001 & $100 \% / \mathrm{L}$ & Late - Need 6-26-97 (Past Dua) & & & & & & & & & \\
\hline 11210.68 & Need Mactine Dwo Compr Dist Sigo.s & 245160 & $6 / 26 / 97$ & $628 / 97$ & & $100 \%$ & & & & $\Delta$ & & & & & & \\
\hline 11210.67 & Appd CPD / Release Dup, Compr Disk Assy Slg 6.s & 245160 & 9/5/97 & 8/5/979 & 0.01 & $100 \%$ & & & & & & & & & & \\
\hline 11210.68 & Need Dwg. Compr Disk Assy Stg 6 - 5 & 245160 & 9/15/97 & $9 / 15 / 97$ & & $100 \%$ & $\ldots . . . .$. & & & & & & & & - & \\
\hline 11210.69 & Complete Aero Design, Compr Blado Stg 7 -S & $245175-1$ & 5/9/97 & $5 / 9197$ & 001 & $100 \%$ & ............ & & & $\Delta$ & & & & & & \\
\hline 11210.70 & Complete Mech Design. Compr Blade Sto $7 \cdot \mathrm{S}$ & $245175-1$ & $5 / 16 / 97$ & $5 / 16197$ & 0.01 & $100 \%$ & $\ldots$ & & & $\Delta$ & & & & & & \\
\hline $11210.70 \mathrm{a}$ & Appd Casting CPD / Duvg, Compr Blade Sto 7 .s & 245176 & $9 / 13 / 97$ & $9 / 13 / 97$ & 001 & $100 \%$ & Lale - Need 4-23-97 (Pasi Dua) & & & & & & & & & \\
\hline $11210.70 \mathrm{~b}$ & Need Casting Dug. Compr Blade Sig 7.5 & 245176 & $4 / 23 / 97$ & $4 / 23 / 97$ & & $100 \%$ & Past Due & & & $\Delta$ & & & & & & \\
\hline 11210.71 & APpd CPD / Dwg Compr Blade St9 $7-5$ & 245175 & $10 / 15 / 97$ & $10 / 15 / 97$ & 0.01 & $0 \% / 1$ & Late - Nead 7-8-97 (Pasl Due) & & & a & & & & & $\cdots$ & \\
\hline 11210.72 & Need Owg Compr Blade Stg 7.5 & 245175 & $78 / 97$ & 781897. & & $100 \%$ & . & & & $\Delta$ & & & & & & \\
\hline 11210.73 & Appd Forging CPD / Owg, Compr Disk Sig 7-S & 245172 & $5 / 8 / 97$ & $5 / 6 / 97$ & 0.01 & $100 \%$ & & & & $\Delta$ & & & & & & \\
\hline 11210.74 & Naed Forging Owg Compr Disk Sig 7 - S & 245172 & $6 / 10 / 97$ & 6/10/97 & & $100 \%$ & & & & $\Delta$ & & & & & & \\
\hline 11210.75 & Appo Machine CPD / Dugg, Compr Disk Sig 7 - S & 245170 & $9 / 5 / 97$ & /1/5/97 & 0.01 & $100 \%$ & Late - Need 6-26-97 (Past Dua) & & & -4 & & & & & & \\
\hline 11210.76 & Need Machine Dwg. Compr Disk Sig 7 - S & 245170 & $6 / 26 / 97$ & 626,97 & & $100 \%$ & 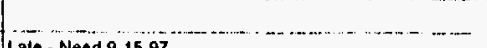 & & & $\Delta$ & & & & & & \\
\hline 11210.77 & Appd CPO I Dug. Compr Disk Assy Stg 7 - S & 245170 & $9 / 5 / 97$ & $\frac{9 / 5 / 97}{0 / 15 / 107}$ & 0.01 & $100 \%$ & Late - Noed 9-15-97 & & & 4 & & & & & & \\
\hline 11210.78 & $\begin{array}{l}\text { Need Dugg, Compr Disk Assy Sto 7-s } \\
\text { Complete Aero Design, Compr Btade Stg 8.5 }\end{array}$ & $\frac{245170}{245191-1}$ & $\begin{array}{l}9 / 15 / 97 \\
5 / 13 / 97\end{array}$ & $\left\{\begin{array}{l}9 / 15 / 97 \\
5 / 13 / 97\end{array}\right.$ & $\begin{array}{rr}0 \\
0.04\end{array}$ & $\begin{aligned} 100 \% \\
100 \%\end{aligned}$ & $-\cdots$ & & & $\Delta$ & & & & & & \\
\hline 11210.80 & Complete Moch Design, Compr Blado Stg 8 - $\mathrm{S}$ & $245191-1$ & $6 / 6 / 97$ & $6 / 6 / 97$ & 0.01 & $100 \%$ & $\cdots$ & & & $\Delta$ & & & & & & \\
\hline 11210.802 & Appd Casting CPD / Dug Compr Blade Stg 8 -S & 245192 & Q/18/97 & 9/18/97 & 0.01 & $100 \%$ & Late - Need 4-30-97 (Past Duo) & & & & & & & & & \\
\hline $11210.80 \mathrm{~b}$ & Need Casting Dwg Compr Blado Stg o.s & 245192 & $4 / 23 / 97$ & $4 / 23 / 97$ & & 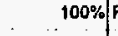 & Past Due & & & $\Delta$ & & & & & & \\
\hline 11210.81 & Appd CPD I Dugo. Compr Biado Sig 8 - 5 & 245191 & $10 / 17 / 97$ & $10 / 17 / 97$ & 001 & $0 \%$ & Late - Need 4-30-97 (Past Dua) & & & $\Delta$ & & & & & & \\
\hline 11210.82 & Nead Dug Compr Blado Stg 8 - S & 245191 & $78 / 97$ & $718 / 97$ & & $100 \%$ / & Pasil Due & & & $\Delta$ & & & & & & \\
\hline 11210.83 & Appo Forging CPD / Dwg, Compr Disk Sig o - s & 245187 & $5 / 1 / 97$ & $5 / 8197$ & 0.01 & $100 \%[$ & & & & $\Delta$ & & & & & & \\
\hline 11210.84 & Need Forging Dwa. Compr Disk Sig 8.5 & 245187 & $6 / 10 / 97$ & $6 / 10 / 97$ & & $100 \%$ & & & & $\Delta$ & & & & & & \\
\hline 11210.85 & Appd Machine CPD / Dwg. Compr Disk Sig \& - S & 245180 & 9/5/97 & $9 / 5 / 97$ & 0.01 & $100 \%$ & Late - Naed 6-26.97 (Past Due) & & & & & & & & & \\
\hline
\end{tabular}

Printed: 10/30/97

Page 3 
Phase 3 and 4

Filter: Total Program

\begin{tabular}{|c|c|c|c|c|c|}
\hline WBS & Task Name & Dwg / Part No & $\begin{array}{l}\text { Start } \\
\text { Dato }\end{array}$ & $\begin{array}{l}\text { End } \\
\text { Date }\end{array}$ & Value \\
\hline 11210.86 & Need Machine Dwg. Compr Disk SL B-S & 245180 & $6 / 26 / 97$ & $6226 / 97$ & \\
\hline 11210.87 & Appd CPD / Releasse Duge, Compr Disk Assy Sig Q - S & 245180 & $915 / 97$ & $9 / 1 / 97$ & \\
\hline$\overline{11210.88}$ & Need Dwa Compr Disk Assy Sig A - S & 245180 & (1515/97 & 9/15/97 & \\
\hline 11210.89 & Complete Aero Design, Compr Blade Stg 9 - 5 & $245205-i$ & 5:66979 & $5 / 6 / 97$ & 0.01 \\
\hline 11210.90 & Complete Mech Design, Compr Blade Slg 9 - S & $245205-1$ & $5 \pi / 97$ & $5 \pi / 97$ & \\
\hline 11210.00 & Appo Casting CPO I Dug, Compor Blado Sto 9-5 & 245206 & $10 / 15 / 97$ & iaj $15 / 97$ & \\
\hline 11210.906 & Need Casting Owg. Compr Blado Sigo.s & 245206 & $4 / 23 / 97$ & $4 / 23 / 97$ & \\
\hline 11210.91 & Appd CPD I I wg Compr Blado Slo $9 . \mathrm{s}$ & 245205 & 1012497 & |10/2497 & \\
\hline 11210.92 & Neod Dug, Compr Blade Sig 9.S & 245205 & $78 / 97$ & 77897 & \\
\hline 121210.93 & Appd Forging Cro i Ouvg Compr 9 Disk St 9 -s & 245202 & 5/8/97 & 5/8/97 & \\
\hline 11210.94 & Nead Forging Dwg. Compr Disk Sig $9-\mathrm{S}$ & 245202 & $6 / 10 / 97$ & $6 / 10 / 97$ & \\
\hline 11210.95 & Appd Machine CPD/OWp, Compr Disk Sig 9 -S & 245190 & 19/5/97 & 9/5/97 & \\
\hline 11210.96 & Need Machine Dwg. Compr Disk Sig 9 - s & 245190 & $6 / 26 / 97$ & 6/26/97 & \\
\hline 11210.97 & Appo CPD I Dwg, Compr Disk Assy Sig $8-S$ & 245190 & $9 / 1 / 97$ & 9/5/97 & \\
\hline 11210.98 & Need Dwg, Compr Disk Assy Sig 9 -s & 245190 & $9 / 15 / 97$ & $9 / 45 / 97$ & \\
\hline 11210.99 & Compleate Aero Design, Compr Blado Sig 10 - S & $245220-1$ & $5 / 8 / 97$ & s/̈̈/97 & \\
\hline 11210.100 & Complete Mech Design, Compr Blade Stg $10 . \mathrm{s}$ & $245220-1$ & $6 / 11 / 97$ & $6 / 1197$ & \\
\hline 11210.1002 & Appa Casting CPD / Dwg, Compr Blada Stg 10 - S & 245221 & 1022297 & $10 / 22 / 97$ & \\
\hline 11210.1006 & Need Casting Dwg. Compr Blade Stg 10.5 & 245221 & $4 / 23 / 97$ & $4 / 23 / 87$ & \\
\hline 11210.101 & Appd CPD I Dug, Compr Blado Sig 10.5 & 245220 & 10,27797 & $10 / 2797$ & 0 \\
\hline 11210.102 & Neod Dwg, Compr Blado SIg $10-\mathrm{S}$ & 245220 & $778 / 97$ & 778997 & \\
\hline 11210.103 & Appo Forging CPD /OWg, Compr An Hub Slg 10-s & 245217 & 9/10/97 & $9 / 10 / 97$ & 0.01 \\
\hline 11210.104 & Need Forging Dwg. Compr An Hub Stg 10 - S & 245217 & $5 / 27197$ & $5 / 27 / 97$ & \\
\hline 11210.105 & Appod Machino CPO / D wog Compr Aft Hub Sig 10 - S & 245200 & $10 / 3197$ & $10 / 3 / 397$ & \\
\hline 11210.106 & Need Machine Durg, Compr A A Hub Stg $10 . \mathrm{S}$ & 245200 & $7126 / 97$ & $7 / 262 / 97$ & \\
\hline 11210.107 & Appd CPD / Owg, Compr An Hub Assy Stg $10-\mathrm{s}$ & 245200 & $100 / 3 / 97$ & $10 / 3 / 97$ & \\
\hline 11210.108 & Need Dugo. Compr Afl Hub Assy Sig 10.S & 245200 & 9/15/97 & $9 / 15 / 97$ & \\
\hline 11210.109 & Apped CPD D Dug, Compr Conter Bolle -5 & 245079 & io/10/97 & $10010 / 97$ & \\
\hline 11210.110 & Neod Dwg, Compr Canter Both - $\mathrm{S}$ & 245079 & 9/16/97 & $8 / 16 / 97$ & \\
\hline 11210.111 & Appd CPD / Owg Compressor Rotor Assy - $S$ & 245070 & $12 / 1 / 997$ & $12 / 8 / 97$ & \\
\hline i1210.112 & Need Dug, Compressoor Rotor Assy -s & 245070 & $10 / 16 / 97$ & $10 / 16 / 97$ & \\
\hline $11210 \cdot 13$ & Complete Assy, Compressor Rotor Assy - $\mathrm{S}$ & $245070-100$ & 3/19/98 & 3/19/98 & \\
\hline 11210.114 & Need, Compressor Rolor Assy -S & $245070-100$ & 3/31/98 & $3 / 31 / 98$ & \\
\hline 11220 & Compressor Casing / Stator Assy - 5 & $245230-100$ & 214/95 & $3 / 31 / 98$ & \\
\hline 11220.1 & Star DLA, Compresssor Housing Assy - 5 & $245231 \cdot 100$ & $4 i 1 / 96$ & $4 / 1 / 196$ & \\
\hline 11220.2 & Appd Casting CPD / Dwg Comprossor Casing Hall - $S$ & 245232 & $111 / 7 / 96$ & 1117198 & \\
\hline 11220.3 & Need Casting Dwg, Compresssor Casing Hall - $\mathrm{S}$ & 245232 & vil7/97 & $3117 / 97$ & \\
\hline 11220.4 & Appd Mach CPD / Owg, Compressor Housing - $S$ & 245231 & $11129 / 97$ & $11 / 29 / 97$ & 1.15 \\
\hline 11220.5 & Need Assy Dwg. Compresssor Housing Assy - $\mathrm{S}$ & 215231 & $6 / 2 / 97$ & $6 / 21297$ & \\
\hline 11220.6 & Complate DLA, VGV Actuation System - S & 245234 & 711197 & 711197 & 0.0 \\
\hline 11220.7 & Appd CPD / Dwg, IGV Split Ring Actuating Assy - S & 245234 & $8 / 18 / 97$ & $8 / 18 / 97$ & 0.16 \\
\hline 11220.8 & Noed Dwg. VGV Split Ring Actualing Assy - 5 & 245234 & 9:15/97 & $9 / 15 / 97$ & \\
\hline 11220.9 & Complete Assy, IGV Splil Ring Actualing Assy - $S$ & $245234 \cdot 100$ & $8 / 18 / 97$ & $8 / 18 / 97$ & 0.1 \\
\hline 11220.10 & Noed Assy, IOV Split Ring Actuating Arsy - S & $245234-100$ & $129 / 98$ & $1 / 29 / 98$ & \\
\hline 11220.11 & ApDd CPD I Dwg, Sig I VGV Split Ring - S & 245241 & $8 / 18 / 97$ & $8 / 18 / 97$ & 0.05 \\
\hline 11220.12 & Need DWg. Sig 1 VGV Split Ring-S & 245241 & 717197 & 771997 & 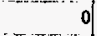 \\
\hline 11220.13 & Appo CPD I Dug, Slg I V GV Split Ring Acluating Assy- & 245235 & $9 / 13 / 97$ & $9 / 13 / 97$ & \\
\hline 11220.14 & Neod Dwg. Stg I VGV Split Ring Aclualing Assy - S & 245235 & 9/15/97 & $9 / 15 / 97$ & \\
\hline 11220.15 & Completo Assy, Sig i VGV Split Ring Acluating Assy-S & $245235-100$ & $9 / 13 / 97$ & Q/1/13/97 & 0.10 \\
\hline
\end{tabular}

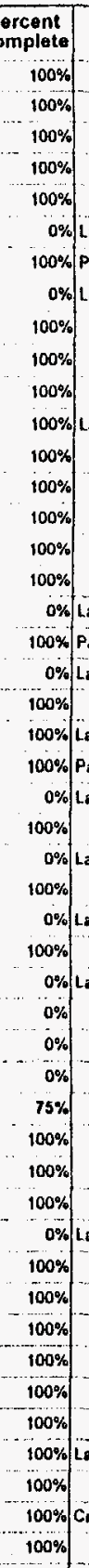

Notes

$100 \%$ Notes

$100 \%$

$100 \%$

0\%/Late - Nead 4-23-97 (Past Dua)

$100 \%$ Pasidue

0\% Lato - Need 7.8.97 (Past Duo)

$100 \%$

$100 \%$

$100 \%$

100\% Lato - Need 6.26.97 (Past Ouo)

$100 \%$

$100 \%$

$100 \%$

$100 \%$

$100 \%$

0\% Late-Neod 4-23-97 (Pasi Due)

100\% Pasi Due

0\% Lalo - Noed 7-8-97 (Past Due) $100 \%$

100\% Lato - Need 5-27-97 (Past Duo)

100\% Past Due

0\% Late - Noed 7.26.97 (Past Dute)

$100 \%$

0\% Late - Need 9-15-97

$100 \%$

0\% Lale - Need 9 - 16.97

$100 \%$

0\% Lato - Need 10-16-97

$0 \%$

$0 \%$

$0 \%$

$75 \%$
$100 \%$

$100 \%$

$100 \%$

0\% Lata - Need 6.2.97 (Pasi Due)

$100 \%$

$100 \%$

$100 \%$
$100 \%$
$100 \%$

$100 \%$

100\% Lale - Need 7.1.97 (Past Due)

$100 \%$

$100 \%$ Crilical - Nood $0-15-97$

$100 \%$

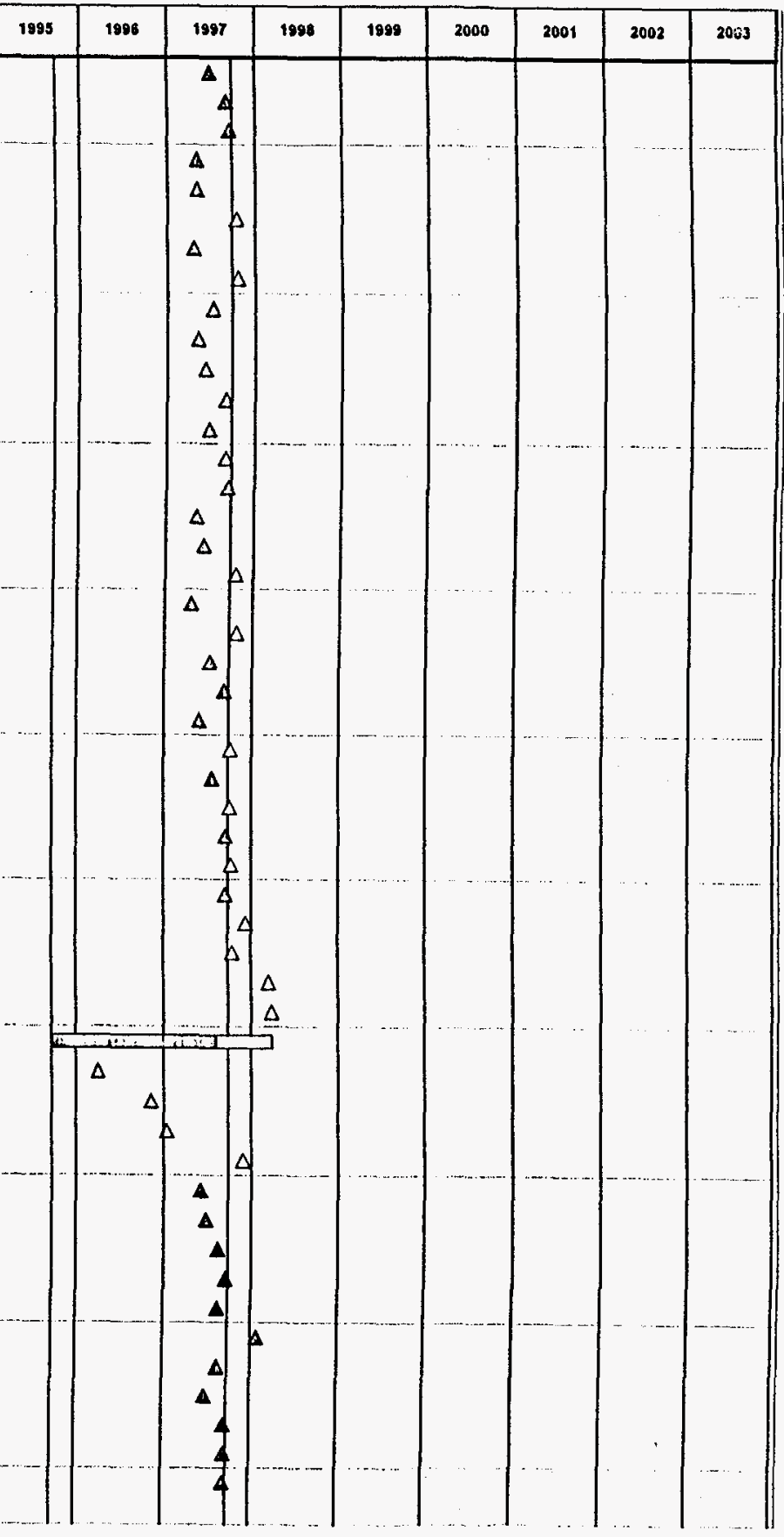

Printed: 10/30/97

Page 4 
Advanced Turbine Systems

Phase 3 and 4

Filter: Total Program

\begin{tabular}{|c|c|c|}
\hline WBS & Task Name & Dwg / Part No \\
\hline 11220.16 & Need Assy, Stg I VGV Split Ring Acluating Assy - 5 & $245235-100$ \\
\hline 11220.17 & Appd CPD I Dwo. 5 tg 2 VGV Spli Ring - S & 245242 \\
\hline 11220.18 & Need Dwg. Stg 2 VGV Splil Ring - S & 245242 \\
\hline 11220.19 & Appd CPD / Dwog, 5192 VGV Spli Ring ACtualing Assy. & 245236 \\
\hline 11220.20 & Need Dwg, SIg 2 VGV Split Ring Actuating Assy - $S$ & 245236 \\
\hline 11220.21 & Complete Assy, Stg 2 VOV Split Ring Acluating Assy - 5 & 245238.100 \\
\hline 11220.22 & Neod Assy, Sig 2 VGV Split Ring Acluating Assy - S & $245236-100$ \\
\hline 11230 & Compressor Stator Assy-s & various \\
\hline 11220.23 & Complete Moch Design, IGV.s & $245250-1$ \\
\hline 11220.24 & Appd Casing CPO / Dwg IGV-S & 245251 \\
\hline 11220.25 & Need Casting Dug. IGV.S & 245251 \\
\hline 11220.26 & Appd Airfoil CPD I OWwg IGV-s & 245250 \\
\hline 112220.27 & Need Airoul Owg. IGV.s & 245250 \\
\hline 11220.28 & Complete Aero Design Stage 1 Compsr Vano - S & $245260-1$ \\
\hline 11220.29 & Complete Mach Design, Stago 1 Compsi Vano -s & $245260-1$ \\
\hline 11220.30 & Appd Casting CPD I Oug. Slage I Compser Vane - 5 & 245261 \\
\hline 11220.31 & Noed Casting Dwg. Slage 1 Compsr Vane - S & $24526 !$ \\
\hline 11220.32 & Appod Airfoil CPO / Dugg, Stage I Compsi Vano - S & 245260 \\
\hline 11220.33 & Need Aiffoll Dug. Stage 1 Compsr Vano - 5 & 245260 \\
\hline 11220.34 & Complete Aero Desion, Stago 2 Compsr Vane - S & $245270-1$ \\
\hline 11220.35 & Compielo Mech Design. Slage 2 Compst Vane - $S$ & $245270-1$ \\
\hline 11220.36 & Appd Casting CPD 10 wo, Stago 2 Compsi Vane - 5 & 245271 \\
\hline $11 \overline{1220.37}$ & Need Casting Dwg. Slage 2 Compsr Vane - 5 & 245271 \\
\hline 11220.38 & Appd Airfoid CPD / Dwg. Stage 2 Compsi Vane - 5 & 245270 \\
\hline 11220.39 & Neod Nirfoil Dwg, Stage 2 Compsr Vane -s & 245270 \\
\hline 1122040 & Completo Aero Dessign, Stago 3 Compsr Stator - $S$ & $245280-100$ \\
\hline 11220.41 & Complete Mech Design, Stage 3 Compst Stator -S & $245280-100$ \\
\hline 11220.42 & Apped CPD I O Owg, Stage 3 Compar Stator Vane - S & 245281 \\
\hline 11220.43 & Need Dug, Stage 3 Compsr Siztor Vano -s & 245281 \\
\hline 11220.44 & Appd CPD I Owg, Stage 3 Compsr Stalor Asty - $S$ & 245280 \\
\hline 1122045 & Need Dwg, Stage 3 Compsr Stator Assy - S & 245280 \\
\hline 11220.46 & Complele Aero Design, Stage 4 Compsr Slator - S & $245285-100$ \\
\hline 11220.47 & Completa Mech Design, Stage 4 Compsr Stator -S & $245285-100$ \\
\hline 11220.18 & Appo CPD / Ourg, Stage 4 Compsr Statoo Vano - S & 245286 \\
\hline 11220.49 & Need Dugh, Stage 4 Compst Stator Vane - S & 245286 \\
\hline 11220.50 & Appd CPO I Oug, Stage 4 Compser Stator Assy - $S$ & 245285 \\
\hline 11220.51 & Neod Dugg, Stage 4 Compsr Stator Assy - $s$ & 245285 \\
\hline 11220.52 & Complete Aero Design. Stage 5 Compst Stator . 5 & $245290-100$ \\
\hline 11220.53 & Complete Mech Dosign, slage 5 Compsr Stator - $S$ & 245290.100 \\
\hline 11220.54 & Appd CPD / Dwg, Stage 5 Compsr Stator Vane - 5 & 245291 \\
\hline 11220.55 & Need Dwo, Stage 5 Compsr Stator Vano - $\mathrm{s}$ & 245291 \\
\hline 11220.56 & Appd CPD I Dwg, Slage 5 Compsr Stator Assy - $s$ & 245290 \\
\hline 11220.57 & Need Owg, Stage 5 Compsr Stator Assy - $S$ & 245290 \\
\hline 11220.58 & Completo Aero Design, Stage 6 Compsr Stator - $S$ & $245295-100$ \\
\hline 11220.59 & Complete Mech Design, Staga 6 Compsr Stator. $S$ & $245295-100$ \\
\hline 11220.60 & Appo CPD I Oung, Stage 6 Comp Stator Vane - $s$ & 245296 \\
\hline 11220.61 & Neod DWg. Stage 6 Comp Stalor Vano - -5 & 245296 \\
\hline 11220.62 & Appd CPO I Dwg, Stago B Comp Stalor Assy - $\mathrm{S}$ & 245295 \\
\hline 11220.63 & Nesd Dwg, Slage 6 Comp Slator Assy - S & 245295 \\
\hline
\end{tabular}

\begin{tabular}{|c|c|}
\hline $\begin{array}{l}\text { Start } \\
\text { Date }\end{array}$ & $\begin{array}{l}\text { End } \\
\text { Date }\end{array}$ \\
\hline $1 / 29 / 98$ & 11/29/88 \\
\hline $9 / 15 / 97$ & $9 / 15 / 97$ \\
\hline 711197 & 711197 \\
\hline $9 / 13 / 97$ & $9 / 13 / 97$ \\
\hline $9 / 15 / 97$ & 9/15/97 \\
\hline 1/15/98 & 18898 \\
\hline $129 / 98$ & $1 / 29 / 80$ \\
\hline I/14/195 & 3/31/88 \\
\hline 2111197 & $2 / 11 / 97$ \\
\hline $8 / 1 / 97$ & B/1/97 \\
\hline $5 / 17797$ & 5/17/87 \\
\hline $111 / 1797$ & $11 / 17 / 97$ \\
\hline |712/97 & $712 / 97$ \\
\hline $8 / 23 / 96$ & $a / 23 / 96$ \\
\hline $9 / 6 / 96$ & $9 / 6 / 96$ \\
\hline $5 / 1197$ & 5/1/197 \\
\hline $5 / 177 / 97$ & $5 / 1797$ \\
\hline 11117797 & $11 / 17 / 97$ \\
\hline $72 / 2197$ & 721297 \\
\hline $9 / 20 / 96$ & $9 / 20196$ \\
\hline $9 / 25 / 96$ & $92 / 25196$ \\
\hline $5 / / 897$ & 518197 \\
\hline $5 / 17 / 97$ & $5 / 17797$ \\
\hline 11117797 & 111171797 \\
\hline 721297 & 72297 \\
\hline $9 / 25 / 96$ & 9/25/96 \\
\hline $10 / / 196$ & $10 / 1196$ \\
\hline $7 / 123 / 97$ & $7723 / 97$ \\
\hline $6 / 1 / 197$ & $6 / 1 / 197$ \\
\hline $8 / 4 / 97$ & $8 / 1497$ \\
\hline 772197 & 772197 \\
\hline $10 / 15 / 96$ & $10 / 15 / 96$ \\
\hline 10122196 & $10 / 22 / 90$ \\
\hline 1723/97 & $7 / 23 / 97$ \\
\hline 6/11978 & $6 / 1 / 197$ \\
\hline $8 / 1 / 497$ & $8 / 14 / 97$ \\
\hline $72 / 97$ & 712197 \\
\hline 51997 & $59 / 97$ \\
\hline $5 / 9 / 97$ & $5 / 9 / 97$ \\
\hline $723 / 97$ & $7 / 223 / 97$ \\
\hline $6 / 1 / 97$ & $6 / 1 / 97$ \\
\hline $811 / 97$ & $8 / 14 / 97$ \\
\hline $7 / 2 / 97$ & 77297 \\
\hline $5 / 5 / 97$ & $3 / 5 / 97$ \\
\hline $5 / 1597$ & $5 / 15 / 97$ \\
\hline $10 / 1 / 97$ & $10 / 197$ \\
\hline 6/1/97 & $6 / 1197$ \\
\hline 101197 & $1011 / 97$ \\
\hline 71297 & 712197 \\
\hline
\end{tabular}
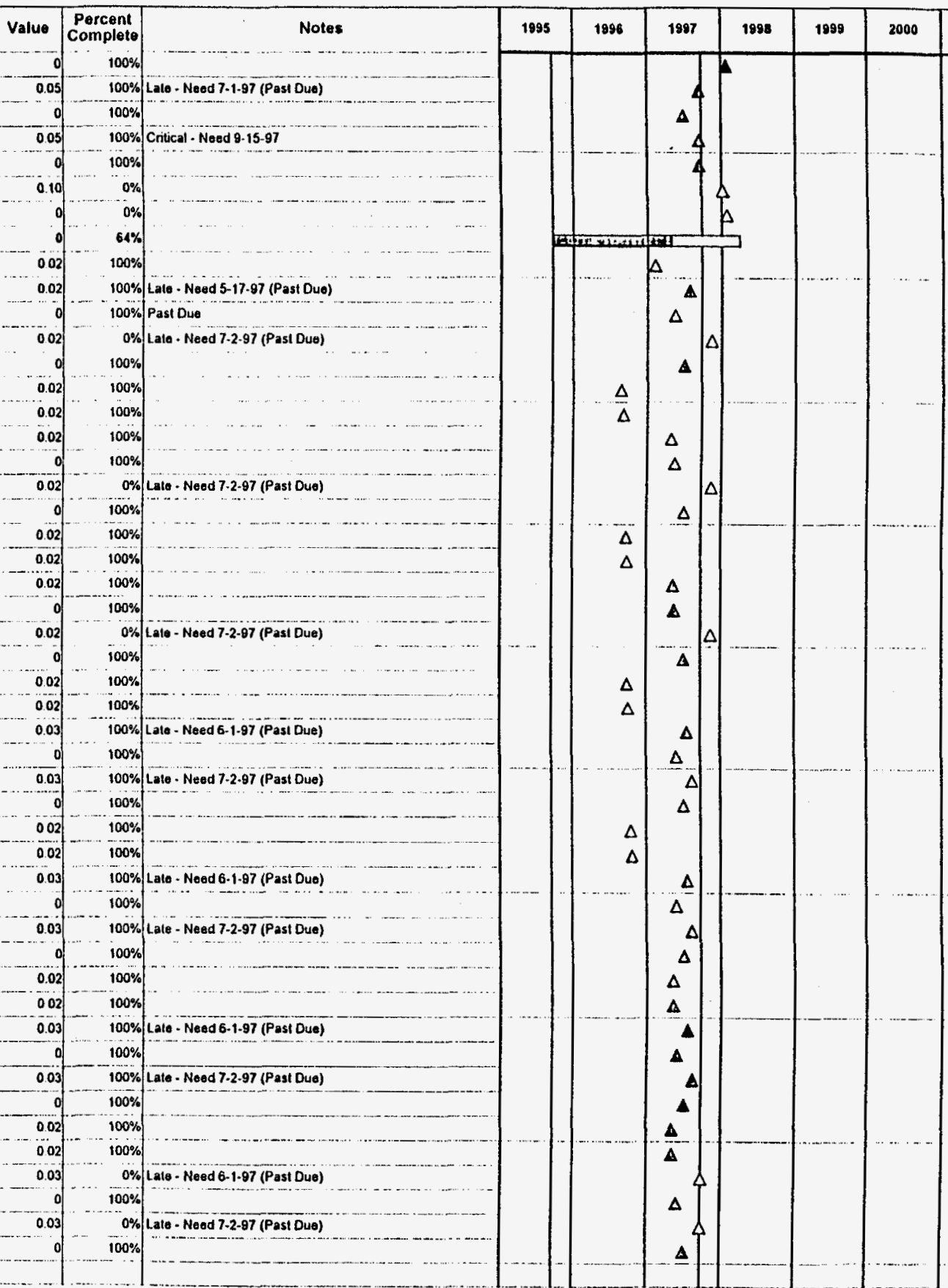

Printed: 10/30/97

Page 5 
Advanced Turbine Systems

Phase 3 and 4

Filter: Total Program

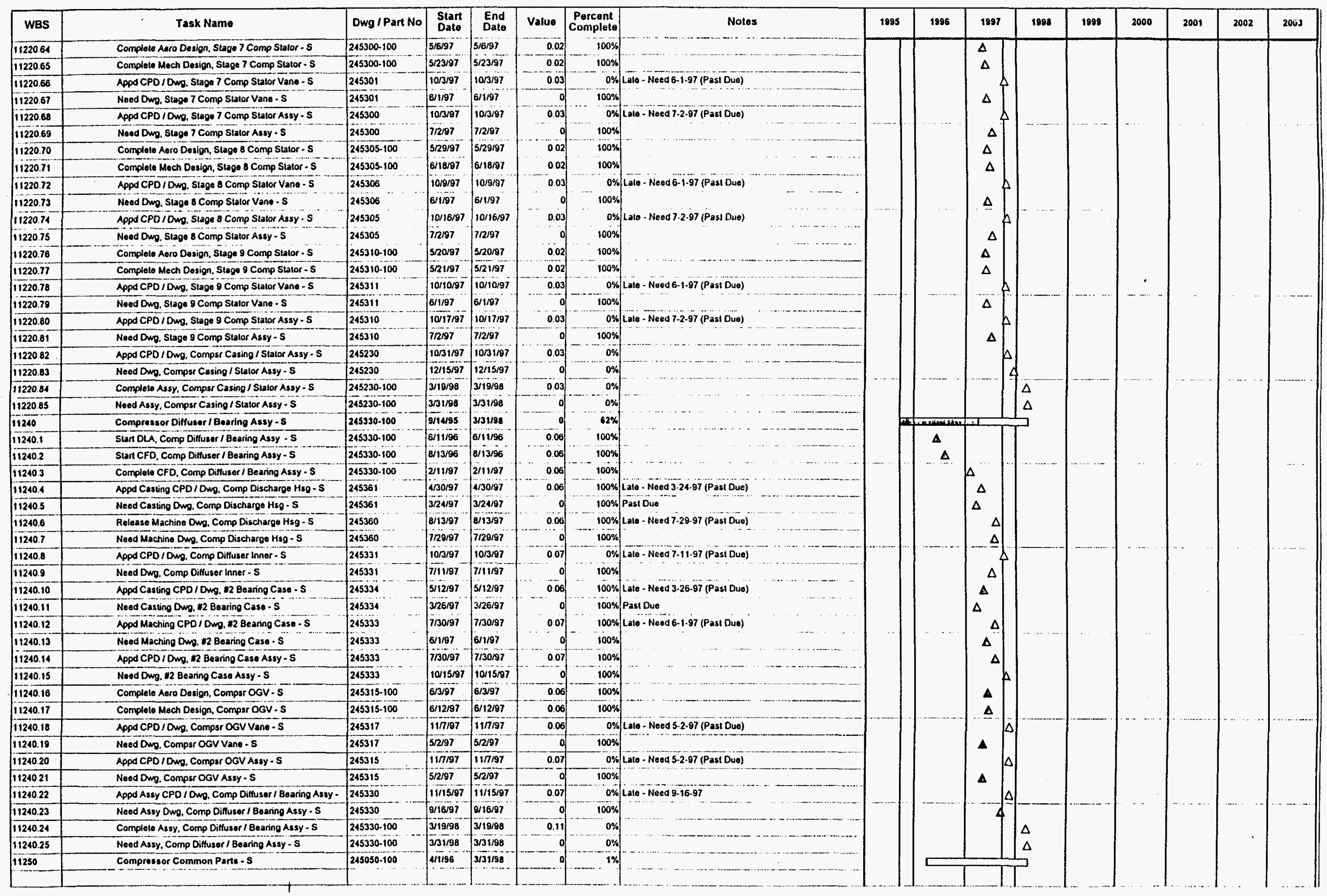

Printed: 10/30/97

Page 6 
Advanced Turbine Systems

Phase 3 and 4

Filter: Total Program

\begin{tabular}{|c|c|c|c|c|c|c|c|c|c|c|c|c|c|c|c|c|}
\hline WBS & Task Name & Dwg / Part No & $\begin{array}{l}\text { Start } \\
\text { Date }\end{array}$ & $\begin{array}{l}\text { End } \\
\text { Dato }\end{array}$ & Value & $\mid \begin{array}{c}\text { Percent } \\
\text { Complete }\end{array}$ & Notes & 1995 & 1996 & 1997 & 1998 & 1899 & 2000 & 2001 & 2002 & 2003 \\
\hline 11250.1 & Appod CPD 1 Dwg Compressor Common Panta - S & $245050-100$ & $121 / 197$ & $12 / 1 / 197$ & 0.75 & $0 \%$ & Laite-Nood 11-30-97 & & & $\Delta$ & & & & & & \\
\hline 11250.2 & Neod Dwg, Compressor Common Parts - $\mathrm{s}$ & $245050-100$ & $11 / 30 / 97$ & $\mid 11 / 30 / 97$ & & $0 \%$ & & & & $\vec{\Delta}$ & & & & & & \\
\hline 11250.3 & Complete, Compressor Common Parts - $s$ & $245050-100$ & $2415 / 98$ & $2 / 15 / 98$ & 0.25 & $0 \%$ & 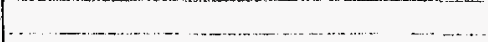 & & & & $\Delta$ & & & & & \\
\hline 11250.4 & Need, Compressor Common Parls - $\mathrm{S}$ & $245050-100$ & $3 / 1 / 188$ & $3 / 31998$ & of & $0 \%$ & & & & & $\Delta$ & & & & & \\
\hline 11300 & Combustor System + S & & $2 / 28 / 96$ & $121 / 1 / 99$ & 0 & $23 \%$ & & & कात & & & & & & & \\
\hline 11310 & Combustor Inlet Scroll (Reqint deleteled) & & $2 / 21 / 196$ & iijoge & & 100\% & $\cdots$ & & $\underline{\underline{a}}$ & & & & & & & \\
\hline 11320 & Combustor Assy- 8 & $245430-100$ & 326696 & [3/31/98 & & $43 \%$ & & & aring & & & & & & & \\
\hline 11320.1 & Complete CFD Combustor Housing - $\mathrm{s}$ & $245450-100$ & $8 / 2196$ & $8 / 2196$ & 003 & $100 \%$ & & & $\Delta$ & & & & & & & \\
\hline 11320.2 & Appd Casting CPD / Dug, Combustor Housing Assy - $\mathrm{S}$ & 245460 & $0 / 13 / 97$ & $8 / 13 / 97$ & 0.05 & $100 \%$ & Late - Need 4/18/97 (Past Dua) & & & $\Delta$ & & & & & & \\
\hline 11320.3 & Need Casting Oung, Combustor Houssing Assy - S & 245460 & 4118197 & $4 / 18 / 97$ & & $100 \%$ & Past Due & & & $\Delta$ & & & & & & \\
\hline 11320.4 & Appd Machine CPD I O Wog. Combustor Housing Assy - $\mathrm{s}$ & 245459 & $9 / 29 / 97$ & $9 / 29 / 97$. & 0.05 & $100 \%$ & (n.................... & & & d. & & & & & & \\
\hline 11320.5 & Need Machine Owg Combustor Housing Assy - $\mathrm{S}$ & 245459 & $10 / 16 / 97$ & $10 / 16 / 97$ & & $100 \%$ & . $\quad$. & & & & & & & & & \\
\hline 113206 & Appd CPD / O wg Combusior Hsg Assy - $\mathrm{S}$ & 245459 & 9/29/97 & $9 / 29 / 97$ & 0.05 & $100 \%$ & & & & 4 & & & & & & \\
\hline $11320 . \overline{7}$ & Need Dwg. Combustor Hsg Assy \& $\mathrm{S}$ & 245459 & $3 / 31 / 98$ & $3 / 31 / 98$ & & $100 \%$ & & & & & $\Delta$ & & & & & \\
\hline $11320.7 \mathrm{a}$ & Appd Casting CPD I Dwg. Combustor End Cover - $\mathrm{s}$ & 245257 & 523997 & $5 / 23 / 97$ & 0.03 & $100 \%$ & Late - Need 3-18.97 (Past Due) & & & $\Delta$ & & & & & & \\
\hline 11320.76 & Need Casting Dwo, Combustor End Cover - $s$ & 245457 & 3/18/97 & $3 / 18 / 97$ & of & $100 \%$ & Past Due & & & $\Delta$ & & & & & & \\
\hline i13320.8 & Appd CPD IOwg Combustor End Cover - $\mathrm{s}$ & 245458 & $103 / 97$ & $10 / 397$ & 0.02 & $0 \%$ & Lalo - Need 6-30-97 (Past Due) & & & $\Delta$ & & & & & & \\
\hline 11320.9 & Need Dwg. Combustor End Cover.s & 245458 & $630 / 97$ & $6 / 30 / 97$ & 0 & $100 \%$ & - & & & $\Delta$ & & & & & & \\
\hline 11320.10 & Stan DLA, Air Diventer Valve Assy - $s$ & $245510 \cdot 100$ & $8 / 21 / 196$ & $8 / 2196$ & 0.02 & $100 \%$ / & (n) & & $\Delta$ & & & & & & & \\
\hline 11320.11 & Appd CPD / Dwo. Air Diverter Valve Assy - $s$ & 245510 & 1013/97 & $10 / 3 / 97$ & 0.05 & $0 \%$ & Late - Need 8-6-97 & & & $\Delta$ & & & & & & \\
\hline 17320.12 & Neod Dwg, Air Divener Valua Assy - S & 245510 & $8 / 6 / 97$ & $86 / 97$ & & $100 \%$ & & & & $\Delta$ & & & & & & \\
\hline 11320.13 & Completo Assy, Air Diventer Valve Assy - S & $245510-100$ & 2118198 & $218 / 198$ & 005 & $0 \%$ & & & & & $\Delta$ & & & & & \\
\hline 11320.14 & Noed Assy, Air Diverier Valve Assy - $\mathrm{S}$ & $245510-100$ & $2 / 18 / 98$ & $2 / 18 / 98$ & 0 & $0 \%$ & $\cdots$ & & & & $\Delta$ & & & & & \\
\hline 11320.15 & Stari DLA. Stage I Diaphragm -s & $245470-100$ & 17197 & $17 / 97$ & 0.03 & $100 \%$ & ............. & & & a & & & & & & \\
\hline 11320.16 & Appo Casting CPD / Owg, Stage 1 Diaphragm - S & 245471 & $1020 / 97$ & $10 / 20 / 97$ & 0.05 & $0 \%$ & Late - Neod 416-97 (Past Due) & & & 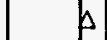 & & & & & & \\
\hline 11320.17 & Need Casting Dwg, Stage I Diaphragm - 5 & 24547 & 416169 & 416697 & o & $100 \%$ & Past Due & & & $\Delta$ & & & & & & \\
\hline 11320.18 & Appd Machine CPD I Dug, Stage I Diaphragm - S & $245470-1$ & $10 / 24197$ & $10 / 24 / 97$ & 0.05 & $0 \%$ & Late - Need 8-15-97 & & & 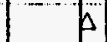 & & & & & & \\
\hline 11320.18 & Need Machine Owg. Stage 1 Diaphragm - 5 & 245470.1 & $9 / 497$ & $9 / 4 / 97$ & & $100 \%$ & & & & 4 & & & & & & \\
\hline 11320.20 & Appd CPO I Owg, Stage I Oiaphragm Assy - $\mathrm{S}$ & $245470-100$ & $10 / 24 / 97$ & $10 / 2497$ & 0.05 & $0 \%$ & & & & $\beta$ & & & & & & \\
\hline 11320.21 & Need Dug. Stage 1 Diaphragm Assy - S & $245470-100$ & 9730197 & $9 / 30187$ & & $100 \%$ & $\ldots$ & & & 8 & & & & & & \\
\hline 11320.22 & Slan DLA, Tutine Preswirler SD 1 - S & $245472-1$ & 1111196 & 1111196 & 0.02 & $100 \%$ & $\ldots . . . . . \ldots \ldots \ldots$ & & & & & & & & & \\
\hline 11320.23 & Appd CPD IO Owg. Piston Ring Preswirler Sig 1 - 5 & 245475 & $10 / 3 / 197$ & $10 / 397$ & 0.05 & $0 \%$ & Late-Neod 4-26.97 (Past Due) & & & $\Delta$ & & & & & & \\
\hline 11320.24 & Need Dwg, Piston Ring Prestwinter Slg 1.S & 245675 & 426697 & 42667 & of & $100 \%$ & Past Due & & & $\Delta$ & & & & & & \\
\hline 11320.27 & Appd Casting CPD I Dwg, Turtino Preswirter Sto 1 - 5 & 245473 & 9/13/97 & $9 / 13 / 97$ & 0.10 & $100 \%$ & Late - Need 7-27-97 (Past Due) & & & 4 & & & & & & \\
\hline 11320.28 & Neod Casting Dug, Turbine Preswirler Sle $1-\mathrm{s}$ & 245473 & 712797 & $7 / 27 / 97$ & & $100 \%$ & & & & $\Delta$ & & & & & & \\
\hline 11320.29 & Appd CPD / Dwg, Turbine Preswirter Sig 1. 5 & 245472 & $10 / 31 / 97$ & $10 / 31 / 97$ & 0.05 & $0 \%$ & Late - Need 7.11-97 (Past Dues) & & & $\Delta$ & & & & & & \\
\hline 11320.30 & Need Dwg. Tuntine Preswirter S1g $1 . \mathrm{S}$ & 245472 & 711197 & 7111197 & 0 & $100 \%$ & & & & $\Delta$ & & & & & & \\
\hline 1132031 & Appd CPD / Dw, Combustor Assy-S & 245450 & $10 / 15 / 97$ & $10 / 15 / 97$ & 005 & $0 \%$ & & & & b. & & & & & & \\
\hline 11320.32 & Need Dug, Combustor Assy - $S$ & 245450 & $11 / 15197$ & $11 / 5 / 97$ & 0 & $0 \%$ & & & & $\Delta$ & & & & & & \\
\hline 11320.33 & Complete Assy, Combustor Housing - $\mathrm{S}$ & $245459-100$ & $10 / 31 / 97$ & $10 / 31 / 97$ & 0.05 & $0 \%$ & $x_{1}+2$ & & & $\Delta$ & & & & & & \\
\hline 11320.34 & Need Assy, Combustor Housing - $S$ & $245459 \cdot 100$ & $3 / 31 / 98$ & $3 / 31 / 98$ & of & $0 \%$ & & & & & $\Delta$ & & & & & \\
\hline 11320.35 & Complete Assy. Combustor Assembiy - $\mathrm{S}$ & 245450.100 & $10 / 15 / 97$ & $10 / 15 / 97$ & 0.15 & $0 \%$ & & & & $b$ & & & & & & \\
\hline 11320.36 & Need Assy, Combustor Assembly - $S$ & $245450-100$ & $3 / 3198$ & $3 / 31988$ & 0 & $0 \%$ & & & & & $\Delta$ & & & & & \\
\hline 11330 & LPM Combustor Liner Assy - $S$ & $245480-100$ & 912869 & $3 / 31198$ & & $75 \%$ & & & 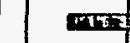 & Ix=ा & & & & & & \\
\hline 11330.1 & Stan DLA, Combustor Liner Assy - S & $245480-100$ & $5 / 221 / 96$ & $5 / 28 / 96$ & 0.10 & $100 \%$ & 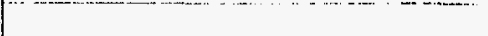 & & $\mathbf{A}$ & & & & & & & \\
\hline i11330.2 & Comploto DLA, Combustor Liner Assy - $\mathrm{s}$ & $245 \times 80-100$ & $5 / 5 / 97$ & $5 / 5197$ & 0.15 & $100 \%$ & & & & $\Delta$ & & & & & & \\
\hline 11330.3 & Appd CPD / Owg Combustor Liner Dolails - S & 245480 & 7725197 & 725197 & 0.25 & $100 \%$ & & & & $\Delta$ & & & & & & \\
\hline 111330.4 & Appd CPD / Dwg. Combuslor Liner Assy - $S$ & 245480 & $9 / 23397$ & $9 / 23 / 97$ & 0.25 & $100 \%$ & Lata - Need 7.7.97 (Past Due) & & & 4 & & & & & & \\
\hline 11330.5 & Need Owg Combustor Liner Assy - S & 245480 & $7 \pi / 97$ & 777197 & & $100 \%$ & $\ldots$. & & & $\Delta$ & & & & & & \\
\hline
\end{tabular}

Printed: 10/30/97

Page 7 
Phase 3 and 4

Filter: Total Program

\begin{tabular}{|c|c|c|c|c|c|c|c|c|c|c|c|c|c|c|c|c|}
\hline WBS & Task Name & Dwg / Part No & $\begin{array}{l}\text { Stant } \\
\text { Date }\end{array}$ & $\begin{array}{l}\text { End } \\
\text { Date }\end{array}$ & Value & $\begin{array}{c}\text { Porcent } \\
\text { Complete }\end{array}$ & Notes & 1995 & 1996 & 1997 & 1998 & 1898 & 2000 & 2001 & 2002 & 2003 \\
\hline 11330.6 & Complate Assy, Combustlor Liner Assy - $\mathbf{S}$ (Rig) & $245480-100$ & $11 / 15 / 97$ & $111 / 15 / 97$ & 025 & $0 \%$ & & & & $\Delta$ & & & & & & \\
\hline 11330.7 & Noed Assy, Combustor Lner Assy - $\mathbf{S}$ (Prod) & $245480-100$ & $3 / 31 / 98$ & $3 / 31 / 18$ & . & $0 \%$ & & & & & $\Delta$ & & & & & \\
\hline 11340 & Ignitor Torch Assy - $\mathrm{S}$ & $245530-100$ & $3 / 1 / 197$ & $51 / 29 / 98$ & 0 & $10 \%$ & & & & एक & 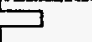 & & & & & \\
\hline 11340.1 & Stan DLA, Ignitor Torch Assy - $\mathrm{S}$ & $245530-100$ & $2 / 20 / 97$ & 228899 & 0.10 & $100 \%$ & & & & & & & & & & \\
\hline 11340.2 & Appd CPD / Dugg, Ignitor Torch Assy - S & 245530 & 1031197 & $10 / 31 / 97$ & 0.40 & $0 \%$ & Lato - Need 4.24-97 (Pasl Due) & & & $\Delta$ & & & & & & \\
\hline 11340.3 & Neod Dwo, Ignitor Torch Assy - $\mathrm{S}$ & 245530 & $4 / 24 / 97$ & 424197 & 0 & $100 \%$ & Past Duo & & & $\Delta$ & & & & & & \\
\hline 11340.4 & Complete Assy. Ionitor Torch Assy . $\mathrm{s}$ & $245530-100$ & $11 / 15 / 97$ & $11 / 15 / 97$ & 0.50 & $0 \%$ & & & & $\Delta$ & & & & & & \\
\hline 11340.5 & Noed Assy, Ignitor Torch Assy - $\mathrm{S}$ & $245530-100$ & $3 / 31 / 98$ & $3 / 31 / 98$ & & & & & & & $\Delta$ & & & & & \\
\hline 11350 & Combustor Oullel Scroll (Reqini deleted) & & $5 / 1 / 96$ & $5 / 15 / 96$ & & $100 \%$ & & & ! & & & & & & & \\
\hline 11360 & Catalytye Combustor Lner Assy - $\mathrm{s}$ & $\cdots$ & $12 / 19 / 96$ & $1211 / 99$ & of & 1\%) & $\cdots$ & & & & & & & & & \\
\hline 11360.1 & Appd CPD / Dwa, Calalylic Liner Assy - S & & $7 / 28 / 98$ & 7128199 & 0.25 & $0 \%$ & & & & & $\Delta$ & & & & & \\
\hline 11360.2 & Select, Catalytic Concept - $S$ & & $12 / 15 / 98$ & $12 / 15 / 98$ & 0.25 & $0 \%$ & & & $\because$. & & & & & & & \\
\hline 11360.3 & Appd CPD / Dwo, Calalyic Liner $S$ & ....... & $3 / 399$ & $3 / 3 / 199$ & 0.25 & $0 \%$ & & & & & & $\Delta$ & & & & \\
\hline 11360.4 & Complete Fab, Calalylic Linar S Rig & $\therefore$ & $121 / 99$ & $12 / 1 / 99$ & & & $\ldots$ & & & & & $\Delta$ & & & & \\
\hline 11400 & Recuperator Section -S & & 9/14/95 & $3 / 31 / 98$ & & 21\% & . & & & & & & & & & \\
\hline 11410 & Recuperator Assembly $\mathrm{s}$ & $245900-100$ & 9/14/95 & $3 / 31 / 98$ & & & 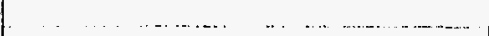 & & & & & & & & & \\
\hline 11410.9 & Appd CPO / DWg Recuperator Assy-S & 245900 & $2 / 15 / 97$ & $2 / 15 / 97$ & 0.20 & $100 \%$ & & & & $\Delta$ & & & & & & \\
\hline 11410.2 & Complete Assy, Recuperator Assy - $S$ (1985) & $245900-100$ & $10 / 15 / 97$ & $10015 / 97$ & 0.30 & & & & & b & & & & & & \\
\hline 51410.3 & Complete Assy, Recuperator Assy - $\mathrm{S}$ & $245900-100$ & $2 / 28 / 98$ & 2128198 & 0.50 & $0 \%$ & 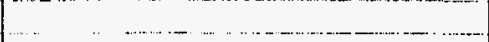 & & & & $\Delta$ & & & & & \\
\hline 11410.3 & Need Assy, Recuperator Assy - $\mathbf{S}$ & $245900-100$ & $3 / 31 / 96$ & $3 / 3198$ & & $0 \%$ & …….............. & & & & $\Delta$ & & & & & \\
\hline 1420 & Recuperstor Piping - $s$ & & 41196 & $2 / 27798$ & & 40\% & $\ldots \ldots$ & & Lesen=2 & & 己 & & & & & \\
\hline 11420.1 & Appd CPD / Dwg, Recup Aif Inlel Assy - S & 245901 & 9/30/97 & $9 / 30 / 97$ & & $100 \%$ & $\ldots$ & & & & & & & & & \\
\hline 11420.2 & Appd CPD I Dwg. Recup Air Oullel Exp Joint Assy - $\mathrm{S}$ & 245902 & $6 / 797$ & an/97. & 0.12 & $100 \%$ & & & & $\Delta$ & & & & & & \\
\hline 11420.3 & Appd CPD I Dug Recup Gas Inlet Flox Ducl Assy -S & 245903 & $6 / 197$ & $6 \pi / 97$ & 0.13 & $100 \%$ & . & & & $\Delta$ & & & & & & \\
\hline 11420.4 & Complete Fab, Recuperator Air Inlot Assy Left - S & $245901-100$ & $2 / 28 / 98$ & $2 / 28 / 98$ & 0.15 & $0 \%$ & ( & & & & $\Delta$ & & & & & \\
\hline 31420.5 & Complete Fab, Recuperator Air Inlet Assy Right - $S$ & $245901-200$ & $2128 / 98$ & 2128198 & 0.15 & $0 \%$ & $\ldots \ldots \ldots$ & & & & $\Delta$ & & & & & \\
\hline 11420.6 & Complete Fab, Recup Air Outtel Exp. Joint Assy - S & $245902-100$ & $2 / 28 / 98$ & $2 / 28 / 98$ & 0.15 & $0 \%$ & $\ldots \ldots . . . . . .$. & & & & $\Delta$ & & & & & \\
\hline 11420.7 & Complete Fab, Recup Gas Inlet Flex Duct Assy -s & $245903-100$ & $2 / 28 / 98$ & $2 / 28 / 98$ & & $0 \%$ & ... $\quad \ldots \ldots \ldots \ldots \ldots$ & & & & $\Delta$ & & & & & \\
\hline 11500 & Gas Fuel Assembiy - S & $245500-100$ & $2 / 28 / 96$ & $11 / 30 / 9$ & & $7 \%$ & $\ldots \ldots \ldots$ & & E.I & & & & & & & \\
\hline 11510 & Fuet Injector Assy - $\mathbf{s}$ & $245515-100$ & 222196 & $3 / 31 / 98$ & of & $25 \%$ & $\ldots$ & & con & & & & & & & \\
\hline 11510.1 & Star DLA, Fuel linjector Assy - 5 & $245515-100$ & $6 / 24 / 96$ & $6 / 2496$ & 0.10 & $100 \%$ & & & $\Delta$ & & & & & & & \\
\hline 11510.2 & Complete CFD, Fuel Injector Assy - $\mathrm{S}$ & $245515-100$ & $5 / 30 / 97$ & 5130197 & 0.15 & $100 \%$ & 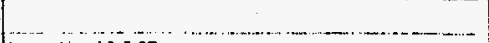 & & & $\Delta$ & & & & & & \\
\hline 11910.3 & Appd CPD I Dug. Fuel linector Assy Fabricated - $\mathrm{s}$ & 246260 & $11 / 28 / 97$ & $11 / 28197$ & 0.25 & $0 \%$ & Late - Need 9-5.97 & & & $\Delta$ & & & & & & \\
\hline 115104 & Need Dwg, Fuel Injector Assy Fabricated - $s$ & 246260 & $915 / 97$ & $9: 5 / 97$ & 0 & $100 \%$ & & & & 4 & & & & & & \\
\hline 11510.5 & Appd CPD / Dwg Gas Fuel Assy - S & 245500 & $10 / 10 / 97$ & $10 / 10 / 97$ & 0.25 & $0 \%$ & . & & & c & & & & & & \\
\hline 131510.6 & Need Dugg Gas Fuel Assy - S & 245500 & $12 / 15 / 97$ & $12 / 15 / 97$ & 0 & $0 \%$ & (6) & & & 4 & & & & & & \\
\hline 11510.7 & Complele Assy, Fuel Injector Assy - $\mathrm{s}$ & $245515-100$ & $11 / 30 / 97$ & $11 / 30 / 97$ & 0.25 & $\%$ & 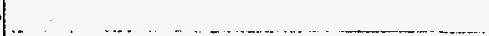 & & & $\Delta$ & & & & & & \\
\hline 11510.8 & Need Assy. Fuel lnjeclor Assy - 5 & 245515.100 & $3 / 31 / 98$ & $3 / 31 / 98$ & of & $0 \%$ & - & & & & $\Delta$ & & & & & \\
\hline 11520 & Fuel Manifolds / Tube Assy - $\mathrm{s}$ & various & $125 / 96$ & $3 / 311 / 98$ & of & 10\% & & & & & & & & & & \\
\hline 11520.1 & Stant DLA, Fuel Manifolds / Tubo Assy - S & Various & $3 / 1 / 97$ & $3 / 1 / 97$ & 0.10 & $100 \%$ & & & & $\Delta$ & & & & & & \\
\hline 11520.2 & Appd CPO / Ougg. Manifold Assy, Gas (Main) - $S$ & 245520 & 10/10/97 & $10 / 10 / 97$ & 0.10 & $0 \%$ & Lale - Need 5/18/97 (Past Due) & & & 1 & & & & & & \\
\hline 11520.3 & Need Dwa, Manifold Assy, Gas (Main) - S & 245520 & $5 / 188 / 97$ & $5 / 18 / 197$ & of & $100 \%$ & & & & $\Delta$ & & & & & & \\
\hline 11520.4 & Appd CPO I Dug Manifold Assy, Gas (Pilol) - S & 245495 & $10 / 10 / 97$ & $10 / 1099$ & 0.10 & $0 \%$ & Lato - Neod 5/18/97 (Pavi Oue) & & & $p$ & & & & & & \\
\hline 11520.5 & Noed Owg Manitold Assy, Gas (Pial) - $\mathrm{S}$ & 245495 & $5 / 18 / 97$ & $5 / 18 / 97$ & of & $100 \%$ & & & & $\Delta$ & & & & & & \\
\hline 11520.6 & Appd CPO I Dwo Tube Assy, Gas (Main) - $S$ & 245521 & $10 / 10 / 97$ & $10 / 10 / 97$. & 0.10 & & - & & & 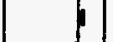 & & & & & & \\
\hline 11520.7 & Noed Dwg, Tube Assy Gas (Main) - $S$ & 245521 & $9 / 15 / 97$ & $9 / 15 / 97$ & & 100\% & ( & & & & & & & & & \\
\hline 11520.8 & Appd CPO / Oug. Tube Assy, Gas (Pilal) - $\mathbf{S}$ & 245526 & $10 / 10 / 97$ & $10 / 10 / 97$ & 0.10 & & 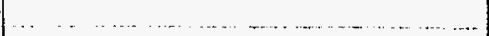 & & & $\Delta$ & & & & & & \\
\hline 11520.9 & Need Dug, Tube Assy, Gas (Pilou) - S & 245526 & 9/15/97 & $9 / 15 / 97$ & at & 100\% & ( & & & & & & & & & \\
\hline 11520.10 & Complete Assy, Manilold Assy, Gas (Main) - $\mathbf{S}$ & $245520 \cdot 100$ & $11 / 15 / 97$ & $11 / 15 / 97$ & 0.15 & & $\ldots \ldots \ldots \ldots$ & & & $\Delta$ & & & & & & \\
\hline
\end{tabular}

Printed: 10/30/97

Page 8 
Advanced Turbine Systems

Phase 3 and 4

Filter: Total Program

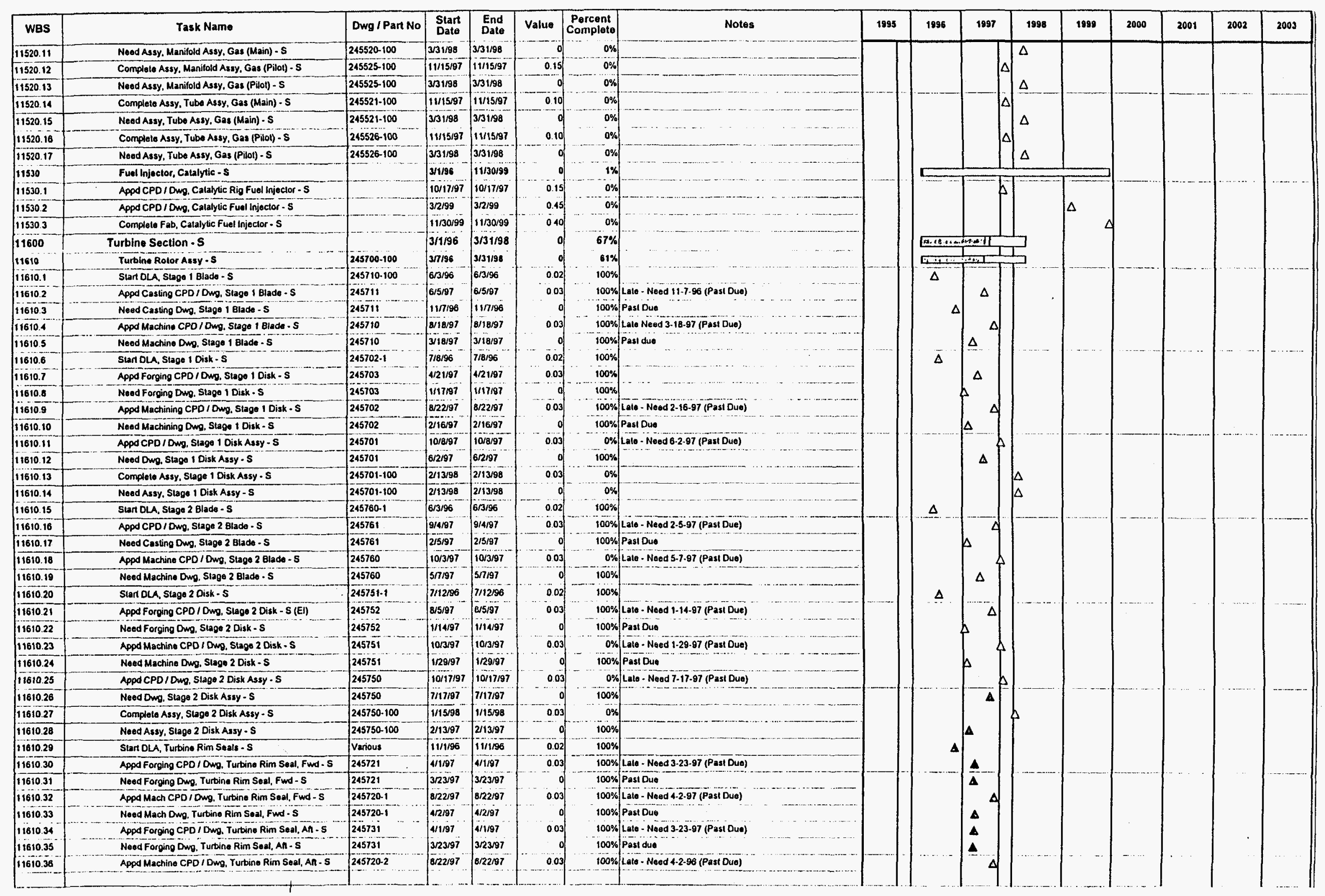

Printed: 10/30/97

Page 9 
Advanced Turbine Systems

Phase 3 and 4

Filter: Total Program

\begin{tabular}{|c|c|c|c|c|c|c|c|c|c|c|c|c|c|c|c|}
\hline WBS & Task Name & Dwg / Part No & $\begin{array}{l}\text { Start } \\
\text { Dato }\end{array}$ & $\begin{array}{l}\text { End } \\
\text { Date }\end{array}$ & Value & \begin{tabular}{|c|} 
Percent \\
Complete
\end{tabular} & 1995 & 1996 & 1997 & 1998 & 1999 & 2000 & 2001 & 2002 & 2003 \\
\hline 11610.37 & Need Mach Dwo. Turtino Rim Seal, An - S & $245720-2$ & 42197 & $4 / 2 / 97$ & 0 & $100 \%$ Pasioue & & & $\Delta$ & & & & & & \\
\hline 11510.38 & Start OLA, Turbing Shan Assy-s & Various & $1114 / 96$ & $1114 / 96$ & 0.02 & $100 \%$ & & $\Delta$ & & & & & & & \\
\hline 11610.39 & Appd Forging CPD / Dwo, Turbino Shafl Assy - $\mathrm{S}$ & 245777 & 72197 & $72 / 97$ & 0.03 & $100 \%$ Lata - Need 2-4-97 (Past Due) & & & $\Delta$ & & & & & & \\
\hline 11610.10 & Need Forging Dwg, Turbine Shafl Assy - $S$ & 245777 & 24197 & $2 / 4 / 97$ & o & $100 \%$ Pasi Due & & & $\Delta$ & & & & & & \\
\hline 11610.41 & Appd Macthine CPD / Owg, Turb. Shan Assy - $S$ & 245776 & $10 / 15 / 97$ & $10 / 15 / 97$ & 003 & $0 \%$ Lata - Need 7.20.97 (Past Due) & & & 4 & & & & & & \\
\hline 11610.42 & Neod Machine Dwo, Turbine Shan Assy - $\mathrm{s}$ & 245776 & 720197 & 720197 & of & $100 \%$ & & & $\mathbf{A}$ & & & & & & \\
\hline 11610.43 & Appd CPD / Dwg, Turtine Shan Assy -S & 245775 & $10 / 15 / 97$ & $10 / 15 / 97$ & 0.03 & $0 \%$ Lato - Noed 8-7-97 (Past Due) & & & 1 & & & & & & \\
\hline 11610.44 & Need Dwg. Turbino Shan Assy -s & 245775 & 887197 & $\mid a / 7 / 197$ & & $100 \%$ & & & $\Delta$ & & & & & & \\
\hline 11610.45 & Appd Forging CPD/ Owg, Turbine Shat Assy, Center - & 245792 & $7124 / 97$ & 724197 & 0.03 & 100\% Late - Need 2-4.97 (Past Duo) & & & $\Delta$ & & & & - & & \\
\hline 11610.46 & Need Forging Dwg. Turbine Shath Assy, Conter -s & 245792 & $2 / 4 / 97$ & $2 / 497$ & & $100 \%$ Past Dus & & & & & & & & & \\
\hline 11610.47 & Appd Machine CPO / Dwg. Turbine Shan Assy, Cenler - & 245791 & $9 \pi / 197$ & $8 / 7 / 97$ & 0.03 & $100 \%$ & & & $\Delta$ & & & & & & \\
\hline 11610.48 & Need Machino Dwg, Turbine Shath Assy, Center - S & 245791 & 7720197 & 7/20/97 & & $100 \%$ & & & $\Delta$ & & & & & & \\
\hline 11610.49 & Appd CPD / Owo, Turtine Shan Assy, Center - $s$ & 245790 & 1002297 & $10 / 22197$ & 0.03 & $0 \%$ Late - Need 8-7.97 (Past Dua) & & & $\Delta$ & & & & & & \\
\hline 11610.50 & Need Dwg, Turtine Shat Assy, Center - $S$ & 245790 & $87 / 97$ & 87/797 & 0 & $500 \%$ & & & $\Delta$ & & & & & & $\cdots$ \\
\hline 11610.51 & Start OLA, Tieboll -S & $245740-1$ & $11 / 4 / 96$ & $11 / 4 / 96$ & 0.02 & $100 \%$ & & $\Delta$ & & & & & & & \\
\hline 11610.52 & Appd CPD I D W T Tieboll - $\mathrm{S}$ & 245740 & 1001599 & $10 / 15 / 97$ & 0.03 & 0\%/Lato - Need 6-17-97 (Past Due) & & & $\theta$ & & & & & & \\
\hline 11610.53 & Need Dwg. Tiebolt - s & 245740 & $6 / 17797$ & 6617997 & of $-1-3-1$ & $100 \%$ & & & $\Delta$ & & & & & & \\
\hline 11610.54 & Star DLA, *3 Beaning Caso Assy - S & $245600-100$ & 1016198 & $10 / 16 / 96$ & 0.02 & $100 \%$ & & $\Delta$ & & & & & & & \\
\hline 11610.55 & Appd Cassing CPD / Dwg, *3 Bearing Case - $s$ & 245807 & 71197 & 7in1997 & 0.02 & $100 \%$ Late - Noed 5.5.97 (Past Dua) & & & $\Delta$ & & ... & & & & \\
\hline 1161056 & Neod Casting Dwg, to Bearing Case - $s$ & 245807 & $5 / 5197$ & $5 / 5 / 97$ & 0 & $100 \% \quad \ldots$ & & & $\Delta$ & & & & & & \\
\hline 1161057 & Appo Machino CPD / Dwg k3 Bearing Case Assy - $S$ & 245806 & $9 / 2697$ & $9 / 26 / 97$ & 0.02 & $100 \%$ Laio - Need 6-16-97 (Past Dua) & & & . & & & & & & \\
\hline 1160.058 & Need Machino Dwg, m3 Boaring Casse Assy .s & 245806 & 630197 & $6 / 30 / 97$ & & $100 \%$ & & & $\Delta$ & & & & & & \\
\hline 11610.59 & Appd CPO IOWg *3 Bearing Case Assy - 5 & 245800 & $101 / 1 / 97$ & $10 / 1 / 197$ & 0.02 & $\% \%$ & & & $\Delta$ & & & & & & \\
\hline 11610.60 & Need Dug wo Bearing Case Assy - $\mathrm{S}$ & 245600 & $10 / 16 / 97$ & $10 / 16 / 97$ & & $0 \%$ & & & & & & & & & \\
\hline 11610.61 & Appd CPD IO Wug Thusti Bearing Assy - $S$ & 245555 & 516197 & $5 / 16 / 97$ & 0.02 & $100 \%$ & & & $\Delta$ & & & & & & \\
\hline 11610.62 & Procuro, Thust Bearing Assy-s & $245555-100$ & $5 / 1097$ & $5 / 10 / 97$ & & ……................. & & & & & & & & & \\
\hline 01610.63 & Appd CPD / Owo. Turbine Rotor Assy - $S$ & 245700 & 10/23/97 & $10 / 23 / 97$ & 0.02 & 0\% Criticat - Nood 10-31.87. & & & a & & & & & & \\
\hline 1161064 & Need Dwo. Turtine Rotor Assy - $\mathbf{s}$ & 245700 & $10 / 31 / 97$ & $10 / 31 / 97$ & & $0 \%$ & & & $\Delta$ & & & & & & \\
\hline 11610.65 & Appd CPD I Owg, Exhaust Collector Inner - $S$ & 245801 & 1009997 & 109997 & 0.02 & $0 \%$ Late - Nood 5.18-97 (Past Due) & & & & & & & & & \\
\hline 11610.68 & Neod Dug. Exhaust Colfiector Inner - S & 245801 & $5 / 18 / 97$ & $5 / 1897$ & 0 & 100\%/Past Due & & & $\Delta$ & & & & & & \\
\hline 11610.67 & Complete Assy, Turbine Rolor Assy - 5 & $245700-100$ & $3 / 19198$ & $3 / 19 / 98$ & 0.03 & $0 \%]$ & & & & $\Delta$ & & & & & \\
\hline 11610.68 & Need Assy, Turbine Rotor Assy - S & $245700 \cdot 100$ & $3 / 31198$ & $3 / 3198$ & of & $\ldots$ & & & & $\Delta$ & & & & & \\
\hline 11620 & Turbine Housing Aszy $-\mathrm{S}$ & & 37196 & 3/31/98 & & $75 \%$ & & & 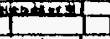 & $\Gamma$ & & & & & \\
\hline 11630 & Turbine Nozxio Assy $s$ & & 311196 & $3 / 31 / 98$ & & $\ldots$ & & Wis & 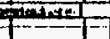 & $\Gamma$ & & & & & \\
\hline 11630.1 & Start OLA, Nozzle Segment Assy Sto 1 -s & 245600.100 & $6 / 3 / 96$ & $6 / 3 / 396$ & 0.05 & $100 \%$ & & $\Delta$ & & & & & & & \\
\hline 116302 & Appd Casling CPO I Dug, Nozzio Segment Stg i - S & 245602 & $5 / 30 / 97$ & $5 / 30097$ & 010 & 100\% Lato - Need 12-23.08 (Past Due) & & & $\Delta$ & & & & & & \\
\hline 116303 & Noed Casting Dwo. Nozzlo Segment Stg i Is & 245602 & $12 / 23196$ & $1223 / 96$ & . & $100 \%$ Pasi Due & & & & & & & & & \\
\hline 11630.4 & Appd Machine CPD / Dug, Nozzlo Segment Stg I - 5 & 245601 & $7330 / 97$ & $7730 / 97$ & 0.05 & 100\% Late - Nood 3-4.97 (Past Due) & & & $\Delta$ & & & & & & \\
\hline 11630.5 & Need Machine Dowp, Nozzlo Segment Stg 1 - $s$ & 245601 & $3 / 497$ & $3 / 4 / 97$ & 0 & $100 \%$ Past duo & & & $\Delta$ & & & & & & \\
\hline 1630.8 & Appd Assy CPD / Dwg. Nozzlo Sogmenl Stg I. S & 245600 & 1011797 & 10171797 & 0.10 & $0 \%$ & & & $b$ & & & & & & \\
\hline 11630.7 & Noed Assy Dwg, Nozzle Segment Sig 1 - 5 & 245600 & $130 / 98$ & $1 / 3099$ & of & $0 \%$ & & & & p & & & & & \\
\hline 11630.8 & Start DLA, Stage 2 Nozzlo Assy - S & $245650-100$ & $7717 / 96$ & 7717796 & 0.05 & $100 \%$ & & $\boldsymbol{A}$ & & & & & & & \\
\hline 11630.9 & Appd Casting CPD / Dwg. Stago 2 Nozzlo Segmont - S & 245652 & $5 / 30 / 97$ & $5 / 30 / 97$ & 0.80 & $100 \%$ Late - Nood 2-21-97 (Past Due) & & & $\Delta$ & & & & & & \\
\hline 11630.10 & Nood Cas ling Dwog, Slago 2 Nozzlo Segment - S & 245652 & 2121197 & 221197 & $?$ & $100 \%$ Pasi Dus & & & $\triangleq$. & & & & & & \\
\hline 11630.11 & Appd Machino CPD I Owg. Slage 2 Nozzlo Sogment - s & 245651.1002200 & $9 / 25 / 97$ & 9/25/97 & 0.05 & $100 \%$ Lale - Noed 7.2.97 (Past Due) & & & 4 & & & & & & \\
\hline 11630.12 & Need Machine Dwa Slage 2 Nozzlo Segment . 5 & $245651-1001200$ & 72197 & $7 / 2 / 97$ & of & $100 \%$ & & & $\Delta$ & & & & & & \\
\hline 11630.13 & Appd CPD / Dugg, Stage 2 Noxzle Assy-S & 245650 & $9 / 25 / 97$ & $9 / 25 / 97$ & 0.05 & $\ldots$ & & & 4 & & & & & & \\
\hline 11630.14 & Noed Dugg. Slago 2 Nozzla Assy -S & 245650 & $10 / 31187$ & $10 / 31 / 97$ & -0 & $100 \%$ & & & $\Delta$ & $\Delta$ & & & & & \\
\hline$\$ 1630.15$ & Completo Assy. Stago 2 Nozzle Assy - S & $245650 \cdot 100$ & $3 / 19 / 88$ & $3 / 19 / 98$ & 0.05 & $0 \%$ & & & & & & & & & \\
\hline
\end{tabular}

Printed: 10/30/97

Page 10 
И əbed

L6/OE/OL :palu!ld

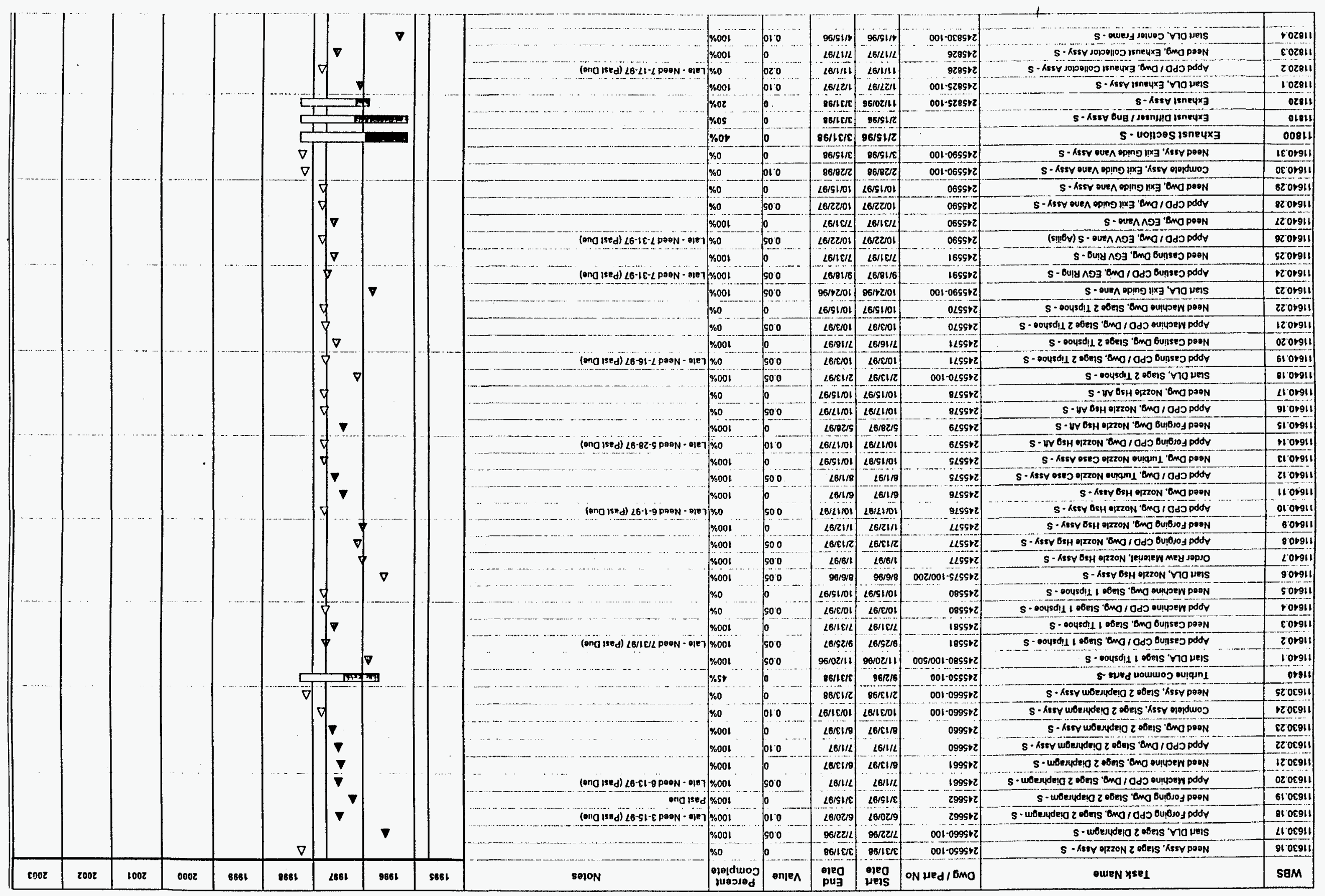

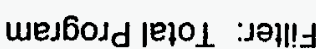

$\rightarrow$ pue $\varepsilon$ әseyd

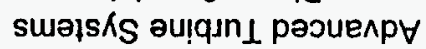


Advanced Turbine Systems

Phase 3 and 4

Filter: Total Program

\begin{tabular}{|c|c|c|c|c|}
\hline WBS & Task Name & Dwg / Part No & $\begin{array}{l}\text { Start } \\
\text { Data }\end{array}$ & $\begin{array}{l}\text { End } \\
\text { Date }\end{array}$ \\
\hline 11820.3 & Appd CPD I Dug, Center Frame - $s$ & 245830 & $10 / 30197$ & $10 / 30 / 97$ \\
\hline 11820.6 & Need Dung Conter Frame -s & 245630 & $5 / 18 / 97$ & $5 / 18 / 97$ \\
\hline 11820.7 & Appd CPD / Dwg, Exhaust Assy - S & 245825 & $111 / 197$ & (111/1/97 \\
\hline 1820.8 & Need Dwg, Exhaust Assy - S & 245825 & $8 / 1797$ & $8 / 177 / 97$ \\
\hline 11820.9 & Complete Assy, Exhaust Assy - s & $245825-100$ & 19999 & $1 / 9 / 98$ \\
\hline 11820.10 & Noed Assy. Exhausi Assy - $S$ & $245823-100$ & $351 / 98$ & 333198 \\
\hline 11900 & Engine Common Parts - S & & $7111 / 96$ & $3 / 25 / 98$ \\
\hline 11910 & Bloed Volve i Duet Asty -s & & $2 / 28 / 97$ & 2/9/98 \\
\hline 11910.1 & Release Final Design, Rolliel Valve 6 Duct Asty - $\mathbf{s}$ & & $10 / 15 / 97$ & $10 / 15 / 97$ \\
\hline 119102 & Complete $\mathrm{Fab}_{\text {a }}$ Rolief Valve \& Duct Assy - $\mathrm{s}$ & & $1212 / 97$ & $12 / 2197$ \\
\hline 11920 & Engine Assembiy Hardware $\mathrm{s}$ & & 711196 & |325/98 \\
\hline 1920.1 & Release Design, Engine Assy Hardware -S & & $\mid 1115597$ & $111 / 15 / 97$ \\
\hline 11920.2 & Complote fab Engino Assy Herdwara - $s$ & & $3 / 25 / 98$ & $3 / 25 / 98$ \\
\hline 12000 & Package Systems -s & & $1211 / 95$ & $12 / 31 / 9$ \\
\hline 12100 & Package Mechanical Systems - S & & $\mid 1211 / 95$ & $4 / 12 / 99$ \\
\hline $121110^{\circ}$ & Generalor - $\mathbf{s}$ & & 1014/96 & 771498 \\
\hline 12110.1 & Release. Generalor Spec.. $S$ & & 71197 & $711 / 97$ \\
\hline 12110.2 & Release, Genarator Procurroment Drawing - $\mathrm{s}$ & & 9/1/97 & 9/1/97 \\
\hline $12 \div 10.3$ & Review, Supplier Genorator Documentation -s & & $10 / 15 / 97$ & $10 / 15 / 97$ \\
\hline 12110.4 & Procure, Generator - s & & 7174198 & 771498 \\
\hline 12120 & Reduction Goarbox -s & & i21/1985 & o129197 \\
\hline 12120.1 & Releasse, Reduction Gearbox Spec. $\mathrm{s}$ & & $3 / 15 / 96$ & $3 / 1 / 1 / 96$ \\
\hline 12120.2 & Complote Supplier Proposala, Geardox -S & & $10 / 16 / 96$ & $10 / 16 / 166$ \\
\hline 12120.3 & Release Po Re Reduction Geartox - $\mathrm{S}$ & & 2/5/97 & $2 / 5 / 97$ \\
\hline 12120.4 & PDR Complete, Dev Roduction Goantox - S & & 771187 & 711197 \\
\hline 12120.42 & PDR Complate, Production Roduction Gearbox - $\mathrm{S}$ & & $101 / 197$ & 10/1/197 \\
\hline 12120.5 & Complete Fab, Dev Reduction Gearbox-s & & $10 / 3197$ & $10 / 11 / 97$ \\
\hline 12120.6 & Completer Assy, Dev. Reduction Geartox - $\mathrm{S}$ & & $111 / 15 / 97$ & iu/1/97 \\
\hline 12120.7 & Receive, Reduction Geartox-s & & 121199 & 124199 \\
\hline 12130 & Electirical Conduit I J-Boxes - - & & 912197 & 3325198 \\
\hline 12130.1 & Release, Electrical Conduit IJ-boxes - $\mathrm{S}$ & & $\mid 10 / 31197$ & $10 / 3197$ \\
\hline 12130.2 & Procure Electrical System Compononis - $s$ & & $3 / 25 / 98$ & $3 / 25198$ \\
\hline 12140 & Package / Gonerstor Frame - 8 & - & $7116 / 97$ & $6 / 22198$ \\
\hline 12140.1 & Reloase, Final Dosign, Packago Frame - 5 & & 9/30/97. & Prsargr \\
\hline 12140.2 & Complete Fab, Pockage Framo - S & & $12115 / 97$ & $12115 / 97$ \\
\hline 12130 & Enclosure Assembly - $\mathrm{s}$ & & 12110197 & 4/12189 \\
\hline 12150.1 & Release final Design, Package Enclosure - $S$ & & $6 / 12 / 98$ & 6/21298 \\
\hline 12150.2 & Procure, Packago Enclosuro - S & & $4 / 12199$ & 41121290 \\
\hline 12160 & Intet Filter I Silencer / Ducting - S & & $9 / 2197$ & 1112199 \\
\hline 12160.1 & Release, Final Design, Inlet Filler - S & & $111 / 13 / 97$ & $11 / 13107$ \\
\hline 12160.2 & Procure / Fab, Inlet Filter - 5 & & $1 / 1 / 2 / 99$ & $11 / 2199$ \\
\hline 12170 & Resuperator Support - $\mathbf{S}$ & & 10/1997 & $5 / 18 / 98$ \\
\hline 12170. & Release, Final Dosign, Rocuperator Support - $S$ & & $7113 / 98$ & $7113 / 98$ \\
\hline 12170.2 & Procure Fab Rocuperator Support -S & & $5 / 18 / 98$ & $5 / 1898$ \\
\hline 12200 & Package Control a Indicating System - S & & $5 / 1 / 96$ & $2 / 14199$ \\
\hline 12210 & Control Console.s & & $51 / 196$ & 212199 \\
\hline 12210.1 & Release, Controis Spec. - S & & $4 / 21 / 97$ & $4 / 21 / 97^{\circ}$ \\
\hline 122102 & Complete Design, On Skid Controller Protatype - 5 & & $11115 / 97$ & $11 / 15 / 97$ \\
\hline 12210.3 & Complete Test in-House OSC.S & & $2728 / 98$ & $21281 / 98$ \\
\hline
\end{tabular}

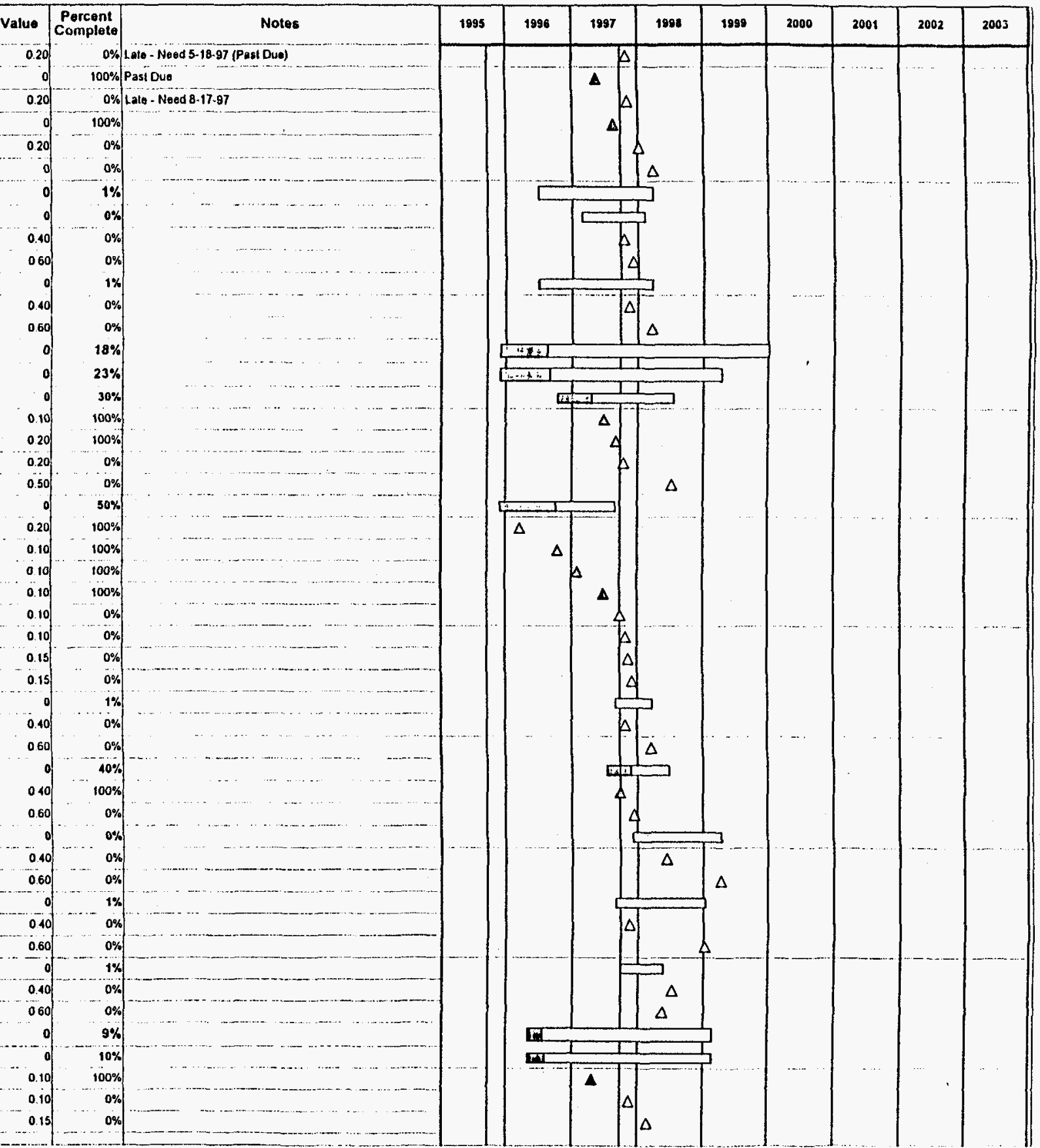

Printed: 10/30/97

Page 12 
Advanced Turbine Syslems

Phase 3 and 4

Filter: Total Program

\begin{tabular}{|c|c|c|c|c|c|c|c|c|c|c|c|c|c|c|c|c|}
\hline WBS & Task Name & Dwg / Part No & $\begin{array}{l}\text { Start } \\
\text { Date }\end{array}$ & $\begin{array}{l}\text { End } \\
\text { Date } \\
\text { pale }\end{array}$ & Value $c$ & $\begin{array}{c}\text { Percent } \\
\text { Complote }\end{array}$ & Notes & 1995 & 1896 & 1997 & 1998 & 1999 & 2000 & 2001 & 2002 & 2003 \\
\hline 17200.2 & Procurs, Balance of Plant - $S$ & & 21899 & 218199 & 0.60 & $0 \%$ & & & & & & $\Delta$ & & & & \\
\hline 18000 & Tooling / Fixtures - $S$ & & $11 / 3 / 95$ & $12 / 1 / 99$ & o) & $39 \%$ & & & & जut & & & & & & \\
\hline 12100 & Cold Section Tooling .5 & & 1113/9s & 3/24/99 & of & $55 \%$ & & & +7 & 宜 & $=$ & 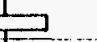 & & & & \\
\hline 10200 & Hot Section Toolling - $\mathrm{S}$ & & $9 / 3 / 96$ & 5/28/99 & of & 40\% & & & En: & Sering & & & & & & \\
\hline 18300 & Rotors Tooling - 5 & & $9 / 3 / 96$ & $1 / 29199$ & & $35 \%$ & & & E & & & & & & & \\
\hline 18400 & Recuperator Tooiling - $s$ & & 10011968 & 3/3i/98 & & $35 \%$ & & & 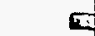 & 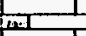 & & & & & & \\
\hline 14500 & Engine Assy Tooling - 5 & & 9/2/97 & 12/1/99 & & $1 \%$ & 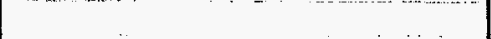 & & & & & & & & & \\
\hline 10700 & Superior Gear Tooling - S & & $9 / 2 / 97$ & 8/31/9a & & 1\%. & & & & & 口 & & & & & \\
\hline 19000 & Technology Development & & 9/14/95 & $12 / 30 / 9$ & 1 & $25 \%$ & & & $x+1$ & & & & & & & \\
\hline 19100 & Advanced Cooling \& Sealing & & $9 / 14 / 95$ & $3 / 15 / 98$ & of. & $57 \%$ & & & MEREGiES & & & & & & & \\
\hline 19110 & Advanced Rotor Sealing (ARS) & & 9/1495 & 2/2198 & & $65 \%$ & & & & & & & & & & \\
\hline 191101 & Complete, Fi Tree Altachment Cooling Definition - ARS & & $726 / 96$ & 726196 & 0.15 & $100 \%$ & Design Into to De sign & & $\Delta$ & & & & & & & \\
\hline 19110.2 & Complete, Rim Soal Flow Optimization Definition - ARS & & $1211 / 97$ & 1211197 & 0.15 & $0 \%$ & Di45 Prionity i Tost Egr Req'd & & & & & & & & & \\
\hline 191103 & Complete Rotor Det, Impingment Cooling Oplimization - & & $12 / 10 / 96$ & $12 / 10 / 96$ & 0.10 & $100 \%$ & ............. & & & & & & & & & \\
\hline 191104 & Complete Build, Test Rig - ARS & & $12 / 17 / 96$ & $12 / 77 / 96$ & 0.15 & $100 \%$ & 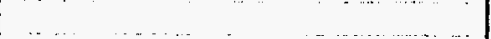 & & & & & & & & & \\
\hline 19110.5 & Rim Seal Design Into to Mech. Design - ARS & & $5 / 1 / 97$ & $5 / 1197$ & 0.15 & 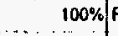 & Rim Seal Design Info 10 Design & & & $\Delta$ & & & & & & \\
\hline 19110.8 & Complete Build, Test Rig - ARS & & 61197 & $6 / 1 / 97$ & 0.10 & $100 \%$ & 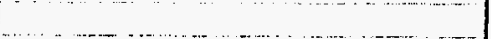 & & & $\Delta$ & & & & & & \\
\hline 19110.7 & Completer, Test Repor Cooling Technologies - ARS & & $12 / 23 / 97$ & $12 / 23 / 97$ & 0.10 & $0 \%$ & . & & & & & & & & & \\
\hline 19110.8 & Complete, Topical Report, Adv Cooling \& Sealing - S\&C & & 22198 & $2 / 2 / 98$ & 0.10 . & $0 \%$ & $-x$ & & & & & & & & & \\
\hline 19120 & Advanced Airtoil Cooling - AAC & & $9 / 11495$ & $12 / 23197$ & & $55 \%$ & 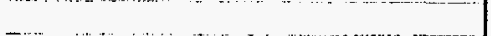 & & & & & & & & & \\
\hline 19120.1 & Complete, Screw Cooting Developmient - MAC & & $6 / 11496$ & $6 / 14 / 96$ & 0.25 & $100 \%$ & 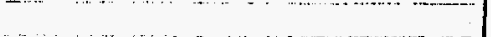 & & $\Delta$ & & & & & & & \\
\hline 19120.2 & Complete, Blade Vortex Cooling Tesi Rig - AAC & & $12 / 13 / 96$ & $12 / 13 / 96$ & 0.25. & $100 \%$ & . & & & & & & & & & \\
\hline 191203 & Completo Topical Repon, Screw Cooling - ACC & & $12 / 15 / 97$ & $12 / 15 / 97$ & 0.10 & $0 \%$ & . & & & & & & & & & \\
\hline 19120.4 & Complete Test Rig, Film Cooling - AAC & 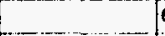 & $6 / 30197$ & $6 / 30 / 97$ & 0.05 & $100 \%$ & 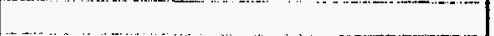 & & & $\Delta$ & & & & & & \\
\hline 19120.5 & Complete Topical Report, film Cooling - ACC & & $12 / 15 / 97$ & $12 / 15 / 97$ & 0.10 & $0 \%$ & & & & & & & & & & \\
\hline 19120.6 & Complete, Cooling Flow Optimization - ACC & & $101 / 197$ & $101 / 197$ & 0.15 & $0 \%$ & 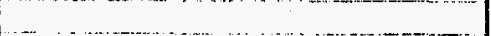 & & & & & & & & & \\
\hline 19120.7 & Completio Topical Report, Cooling Flows - ACC & & $12 / 31 / 97$ & $12 / 31 / 97$ & 0.10 & $0 \%$ & . & & & & & & & & & \\
\hline 19130 & Tip Clearance Control - TCC & & 9211495 & 9/301978 & & 40\% & - n & & inter & & & & & & & \\
\hline 19130.1 & Completer, Satum Concept Selection - ICC & & $4415 / 96$ & $4 / 15 / 96$ & 0.20 & $100 \%$ & 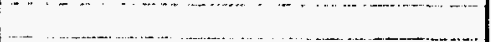 & & $\bar{\Delta}$ & & & & & & & \\
\hline 19130.2 & Complete Build, Satum Engine - TCC & & 2115198 & $2115 / 98$ & 0.30 & $0 \% / s$ & Slipped due 10 Test Bed Availability & & & & $\Delta$ & & & & & \\
\hline 19130.3 & Complete. Salum Test Report - TCC & & $3 / 15 / 98$ & $3 / 15 / 98$ & 0.30 & $0 \%$ & . & & & & $\Delta$ & & & & & \\
\hline 19130.4 & Complete, Mars Concepi Selection - TCC & & $5 / 110 / 96$ & $5 / 10 / 96$ & 0.20 & $100 \%$ & - & & $\Delta$ & & & & & & & \\
\hline 19200 & Low Emission Combustion & & $9 / 14 / 95$ & $6 / 30 / 99$ & 0 & $47 \%$ & & & 5 & iI & & & & & & \\
\hline 19210 & Ultra Lean Promix (ULP) & 7 & 9 gric/9s & $12223 / 97$ & o. & $50 \%$ & & & ning & & & & & & & \\
\hline 19210.1 & Release Prelim Design, Injector Assy - ULP & & $2 / 16 / 96$ & $2116 / 96$ & 0.10 & $100 \%$ & 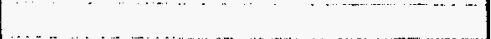 & & $\Delta$ & & & & & & & \\
\hline 19210.2 & Start Test, Irjector Assy. Single Can - ULP & & $5 / 115 / 96$ & $5 / 15 / 96$ & 0.15 & $100 \%$ & - & & $\Delta$ & & & & & & & \\
\hline 19210.3 & Comptete Selection, injector Concept - ULP & & $7715 / 96$ & $7115 / 96$ & 0.15 & $100 \%$ & & & $\Delta$ & & & & & & & \\
\hline 19210.4 & Complale Redesign, Injeclar Assy - ULP & & $4 / 1 / 97$ & 41197 & 0.10 & $100 \%[$ & Completed without redesign & & & $\Delta$ & & & & & & \\
\hline 19210.5 & Complate Tost, Single Injector - ULP & & 10/30/97 & $10 / 30 / 97$ & 0.15 & $0 \%$ & . & & & $\ldots$ & & & & & & \\
\hline 19210.6 & Complete Test, Combustor Liner - ULP & & $10 / 30 / 97$ & $10 / 30197$ & 0.15 & $0 \%$ & & & & & & & & & & \\
\hline 19210.7 & Complate Topical Repor, ULP Prool of Concepl - ULP & & $12 / 30 / 97$ & $12 / 30 / 97$ & 0.20 & $0 \%$ & $+\infty$ & & & & & & & & & \\
\hline 19220 & Calalytic Combustion (CAT) & & $9 / 1495$ & $6 / 30199$ & of. & $35 \%$ & 1. & & & & & & & & & \\
\hline 19220.1 & Start Design, Single Can Catalyvic Combustor Rig - CAT & & 8911495 & $9 / 14 / 95$ & $0.80]^{-}$ & $100 \%$ & -1 & & & & & & & & & \\
\hline 19220.2 & Stan Tests, Single Can Catalytic Combustion Rig - CAT & & $8 / 30 / 96$ & $\mathbf{3 3 0 / 9 8}$ & 0.10 & $100 \%$ & 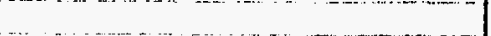 & & $\Delta$ & & & & & & & \\
\hline 19220.3 & Complete Tesis, Single Can Catatric Combustion Rig - & & $12 / 31 / 96$ & $1231 / 96$ & 0.15 & $100 \%$ & 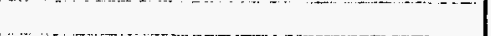 & & & & & & & & & \\
\hline 19220.4 & Complete Design, Calatyic Combustor - CAT & & 1230497 & 1230197 & 0.15 & $0 \%$ & 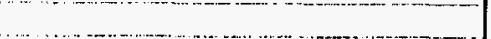 & & & & & & & & & \\
\hline 19220.5 & Complate Engine Assy, Catalycic Combustor. CAT & & $8 / 30198$ & (a/30/98 & 0.15 & $0 \%$ & (n) & & & & $\Delta$ & & & & & \\
\hline 19220.6 & Complate Tests, Calatydic Combustor - CAT & & $3 / 30 / 99$ & 330199 & 0.15 & $0 \%$ & & & & & & $\Delta$ & & & & \\
\hline 19220.7 & Complate Topical Report, Low Emission Combustion & & $6 / 30 / 99$ & $6 / 30 / 99$ & 0.20 & $0 \%$ & (1) & & & 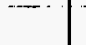 & & $\Delta$ & & & & \\
\hline
\end{tabular}

Printed: 10/30/97

Page 14 
Advanced Turbine Systems

Phase 3 and 4

Filter: Total Program

\begin{tabular}{|c|c|c|c|c|c|c|c|c|c|c|c|c|c|c|c|c|}
\hline WBS & Task Name & Dwg / Part No & $\begin{array}{l}\text { Start } \\
\text { Date }\end{array}$ & $\begin{array}{l}\text { End } \\
\text { Date }\end{array}$ & Value & $\begin{array}{c}\text { Percent } \\
\text { Complete }\end{array}$ & Notes & 1895 & 1996 & 1997 & 1990 & 1899 & 2000 & 2001 & 2002 & 2003 \\
\hline 12210.4 & Retease, Control Schematic / Wiring Orawing - 5 & 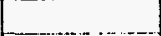 & $7118 / 98$ & 711898 & 0.10 & $0 \%$ & & & & & $\Delta$ & & & & & \\
\hline 12210.5 & Assemblo, Control Consola - S & & $1215 / 98$ & $1215 / 98$ & 0.15 & $0 \%$ & & & & & $\Delta$ & & & & & \\
\hline 12210.6 & Complete, Control Console Slatic Tost - $S$ & & 211499 & 211498 & 0.25 & $0 \%$ & & & & & & $\Delta$ & & & & \\
\hline 12210.7 & Complete Design, On Skid Controllar - $S$ & & $2 / 14199$ & 2141499 & 0.15 & $0 \%$ & & & & & & $\Delta$ & & & & \\
\hline 12220 & Lube Ofl Sysiem * 5 & & $5 / 1 / 96$ & $6 / 1 / 98$ & of & $1 \%$ & ....................... & & L & & & & & & & \\
\hline 12220.1 & Reloase, Lube Oil System Schiomatic - $S$ & & $10 / 15 / 97$ & $10 / 15 / 97$ & 0.25 & $0 \%$ & 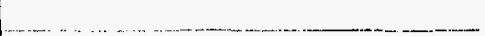 & & & 4 & & & & & & \\
\hline 12220.3 & Releasso, Lube Oil Piping - $\mathrm{s}$ & & $4115 / 98$ & $4 / 15 / 98$ & 0.25 & $0 \%$ & & & & & $\Delta$ & & & & & \\
\hline 12220.3 & Procure, Lube Oit System Components -s & & 6/8/98 & $6 / 8 / 98$ & 050 & $0 \%$ & & & & & $\Delta$ & & & & & \\
\hline 12230 & Package Fuel System-S & & 70/19/96 & 9:23398 & of & $15 \%$ & & & & & & & & & & \\
\hline 12230.1 & Reloaso. Fuel System Schematic -s & $-\ldots$ & $3 / 25 / 97$ & $3 / 25 / 97$ & 0.15 & $100 \%$ & (n) & & & $\Delta$ & & & & & & \\
\hline 12230.2 & Procure, Fuel System Componets - 5 & & $5 / 18 / 98$ & $5 / 18 / 98$ & 0.25 & $0 \%$ & ......... & & & & $\Delta$ & & & & & \\
\hline 12230.3 & Release Fuel Piping - $s$ & & 7177198 & $7 i 1798$ & 0.25 & $0 \%$ & 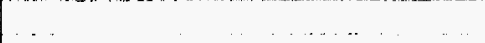 & $\cdots$ & & & $\Delta$ & & & & & \\
\hline 122304 & Complete Fab, Lubo \& Fuel Piping-s & & $9 / 23 / 98$ & $9 / 23 / 98$ & 0.35 & $\%$ & $\cdots$ & & & & & & & & & \\
\hline $12240^{-}$ & start system -s & & $1211 / 96$ & $6 / 22198$ & 0 & $1 \%$ & s. & & & & $\square$ & & & & & \\
\hline 122401 & Release Final Design, Start Systiom - S & & $10 / 15 / 97$ & $10 / 15 / 97$ & 0.40 & $\%$ & ........... & & & s & & & & & & \\
\hline 12240.2 & Procure, siar System Componenis - $s$ & & $6 / 22 / 98$ & $6 / 22 / 98$ & 0.60 & $\%$ & 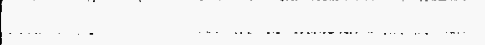 & & & & $\Delta$ & & & & & \\
\hline 12300 & Package Build - $S$ & $\ldots \ldots$ & $3 / 16 / 98$ & $12131 / 99$ & & $0 \%$ & (n) & & & & & & & & & \\
\hline 12300.1 & Crilical Design Review (CDR) -S & & $5 / 29 / 98$ & $5 / 29 / 98$ & 0.04 & $0 \%$ & $\ldots \ldots+\ldots-\ldots$ & & & & $\Delta$ & & & & & \\
\hline 12300.2 & Develop, Package Build Book $\mathbf{S}$ & & $8 / 21198$ & $8 / 2198$ & 0.02 & $0 \%$ & 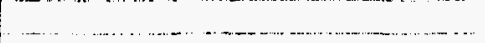 & & & & $\Delta$ & & & & & \\
\hline 12300.3 & Review, Preproduction Dessign - $\mathrm{S}$ & & $\approx / 28198$ & $8 / 28 / 98$ & 0.03 & $0 \%$ & 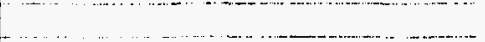 & & & & & & & & & \\
\hline 12300.4 & ACS, Package / Engine $* 3$ (CFE) - S & - & $4115 / 98$ & $415 / 98$ & 0.03 & $0 \%$ & & & & & & & & & & \\
\hline 12300.5 & Package Cash, Packago / Engino *3 (CFE).S & $\ldots$ & $923 / 198$ & $9 / 23 / 98$ & 0.04 & $0 \%$ & - & & & & $\Delta$ & & & & & \\
\hline 12300.6 & Ship, Packego / Enging * 3 (CFE) -S & & 2128199 & $2128 / 99$ & 0.06 & $0 \%$ & {$[-1$,} & & & & & $\Delta$ & & & & \\
\hline 123007 & ACS, Packago / Engine * 4 (CFE) -S & 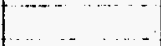 & $6 / 16 / 98$ & $6 / 16 / 98$ & 003 & $0 \%$ & 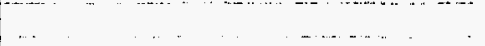 & & & & $\Delta$ & & & & & \\
\hline 12300.8 & Packago Cash, Packago / Engine 4 ( (CFE) - s & & $12 / 1 / 98$ & $12 / 1 / 198$ & 0.04 & $0 \%$ & 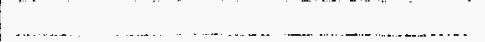 & & & & $\Delta$ & & & & & \\
\hline 12300.9 & Ship. Packago / Engine :4 (CFE) -S & & $4130 / 99$ & 13099 & 0.06 & $0 \%$ & $\mid$ & & & & & $\Delta$ & & & & \\
\hline 12300.10 & ACS, Package / Engine o 5 (CFE)-S & $\ldots \ldots$ & $8 / 14498$ & $8 / 1498$ & 0.03 & $0 \%$ & $\ldots+n$ & & & & $\Delta$ & & & & & \\
\hline 12300.11 & Package Cash, Package / Engine it 5 (CFE) - S & & $2 / 5 / 99$ & $2 / 5 / 99$ & 0.04 & $0 \%$ & $\ldots$ & & & & & $\Delta$ & & & & \\
\hline 12300.12 & Ship, Package I Engine 5 (CFE) - S & $\ldots$ & $6 / 30 / 99$ & $6 / 30 / 99$ & 0.06 & $0 \%$ & $\ldots \ldots$ & & & & & $\Delta$ & & & & \\
\hline 12300.13 & ACS, Package / Engino 6 (CFE) - 5 & & $10 / 16 / 98$ & $10 / 16 / 90$ & 0.3 & $0 \%$ & $\begin{array}{lll}(\ldots \ldots . & \ldots & \ldots\end{array}$ & & & & $\Delta$ & & & & & \\
\hline 12300.14 & Package Cash, Packago / Engine of 6 (CFE) - $\mathrm{S}$ & & $48 / 99$ & $4 / 1 / 99$ & 004 & $0 \%$ & 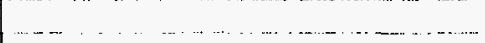 & & & & & $\Delta$ & & & & \\
\hline 1230015 & Ship, Package / Engine 6 (CFE) - S & & $8 / 31 / 99$ & $8 / 31 / 99$ & 006 & $0 \%$ & $(-1.2-1.2$. & & & & & $\Delta$ & & & & \\
\hline 12300.16 & ACS, Package / Engine 7 (CFE) - $\mathrm{s}$ & & $12 / 18 / 98$ & $12 / 18 / 98$ & 0.03 & $0 \%$ & & & & & 4 & & & & & \\
\hline 12300.17 & Package Cash, Package / Engine \& 7 (CFE)-S & & $60 / 999$ & $6 / 9 / 99$ & 0.04 & $0 \%$ & & & & & & $\Delta$ & & & & \\
\hline 12300.18 & Ship, Package / Engina 7 (CFE) - S & & $10 / 31 / 99$ & $10 / 31 / 99$ & 0.06 & $0 \%$ & . & & & & & $\triangle$ & & & & \\
\hline 12300.19 & ACS, Package I Engine $*$ 2(DOE) -S & & $1 / 21 / 99$ & $1 / 21 / 99$ & 0.03 & $0 \%$ & & & & & & & & & & \\
\hline 12300.20 & Package Cash, Package / Engina \# 2 (DOE) - S & & $76 / 199$ & $716 / 99$ & 0.04 & $0 \%$ & ........... & & & & & $\Delta$ & & & & \\
\hline 12300.21 & Ship, Package / Engine $\$ 2$ (OOE) - S & & $11 / 30 / 99$ & $111 / 30 / 99$ & 0.06 & $0 \%$ & 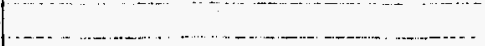 & & & & & 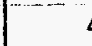 & & & & \\
\hline 12300.22 & ACS. Packago / Engine \& 8 (CFE) - S & & 211799 & $2 / 17799$ & 0.03 & $\%$ & 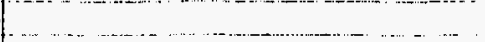 & & & & & & & & & \\
\hline 12300.23 & Packogo Cash, Package / Engine o 8 (CFE) - S & ........ & $8 / 2 / 99$ & $8 / 2199$ & 0.04 & $0 \%$ & & & & & & $\Delta$ & & & & \\
\hline 12300.24 & Ship, Package / Engine \& 8 (CFE) -S & & $12 / 31 / 99$ & $1231 / 199$ & 0.06 & $0 \%$ & 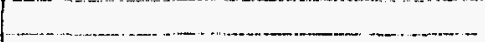 & & & & & & & & & \\
\hline 12400 & O\& M Manual / Mlustrated Parts Liat - $\mathrm{S}$ & & 101197 & 112999 & 0 & $1 \%$ & & & & & & & & & & \\
\hline 12400.1 & First Draft, O\& M Manual - 5 & & $3 / 1 / 98$ & $3 / 31 / 98$ & 0.40 & $0 \%$ & & & & & $\Delta$ & & & & & \\
\hline 124002 & Publish O \& M Manual .S & & $1 / 2999$ & $1 / 29 / 99$ & 0.60 & $0 \%$ & & & & & & s & & & & \\
\hline 17000 & Balance Of Plant - $\mathrm{s}$ & & $9 / 14 / 95$ & $7 / 28 / 99$ & 0 & $1 \%$ & & & & & & & & & & \\
\hline 17100 & Reference Plant Standardization & ….... & $9 / 1495$ & $5 / 15 / 97$ & 0 & $\%$ & & & & מ & & & & & & \\
\hline 17100.1 & Complete, Reference Plant Standardization & $\ldots \ldots-$ & $10 / 15 / 97$ & $10 / 15 / 97$ & 100 & $0 \%$ & Need to confirm w/ Fluor Daniel & & & $\Delta$ & & & & & & \\
\hline 17200 & Balance of Plant - $S$ & & $9 / 299$ & 7128139 & o & $1 \%$ & & & & & & & & & & \\
\hline 17200.1 & Release, Batance of Plant Dasign $\mathrm{S}$ & (-) & $12 / 24 / 97$ & $12 / 24 / 97$ & 0.40 & $0 \%$ & 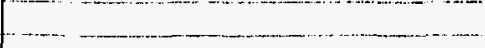 & & & & & & & & & \\
\hline
\end{tabular}

Printed: 10/30/97

Page 13 
Phase 3 and 4

Filter: Total Program

\begin{tabular}{|c|c|c|c|c|c|c|c|c|c|c|c|c|c|c|c|c|}
\hline WBS & Task Name & Dwg / Part No & $\begin{array}{l}\text { Start } \\
\text { Date }\end{array}$ & $\begin{array}{l}\text { End } \\
\text { Date }\end{array}$ & Value & $\begin{array}{c}\text { Percent } \\
\text { Complete }\end{array}$ & Notes & 1995 & 1896 & 1997 & 1998 & 1999 & 2000 & 2001 & 2002 & 2003 \\
\hline 19230 & Advanted Combustion Controls & & $112 / 96$ & $10 / 30 / 97$ & & $75 \%$ & & & & 단. & & & & & & \\
\hline 19230.1 & Complete, Injector Seal Test - Var Geomotry & & $5 / 13 / 98$ & $5 / 3 / 13 / 96$ & 0.15 & $100 \%$ & & & $\Delta$ & & & & & & & \\
\hline 192302 & Complate, Syslem Definition - Co Sensor & & $5 / 30 / 96$ & $5 / 30 / 96$ & 0.10 & $100 \%$ & & & $\Delta$ & & & & & & & \\
\hline 19230.3 & Complele, Loop Test - Var Geomely & & $6 / 30 / 96$ & $6 / 30196$ & 0.10 & $100 \%$ & & & $\Delta$ & & & & & & & \\
\hline 19230.4 & Complete, Veniable Geometry Controls & & $11 / 30 / 96$ & $11130 / 96$ & 0.15 & $100 \%$ & & & $\Delta$ & & & & & & & \\
\hline 18230.5 & Complete, Loop Tost - CO Sensor & & $5 / 23 / 97$ & $5 / 23197$ & 0.25 & $100 \%$ & Slipped due to Test Bod Availability & & & $\Delta$ & & & & & & \\
\hline 19230.6 & Complete, CO Sensor Controls & & $10 / 30 / 97$ & 10/30/97 & 0.25 & $0 \%$ & & & & $\Delta$ & & & & & & \\
\hline 19210 & Advanced Liner Coolling (ALC) & & $1 / 2 / 96$ & $12 / 23 / 97$ & of & $29 \%$ & & & NE=1 & & & & & & & \\
\hline 19240.1 & Complete Topical Repon, Low Emission Combustion & & $12 / 23 / 97$ & $122323 / 97$ & 1.00 & $0 \%$ & - & & & & & & & & & \\
\hline 19300 & Coramic \& Composite Materials Dov. & & 9/144/95 & $10 / 29 / 9$ & 0 & $16 \%$ & & & EntII & & & & & & & \\
\hline 19310 & Ceramic Combustor Materials Dev. & & 4i1/96 & $1223 / 98$ & o & $25 \%$ & . $\quad \ldots \ldots . . . .$. & & क्षा & & & & & & & \\
\hline 19310.1 & Fab, Prolotype Rings - Combustor & & $8 / 31 / 97$ & | $8 / 31 / 97$ & 0.25 & $100 \%$ & - & & & 4 & & & & & & \\
\hline 19310.2 & Complate, Combustor Matarials Dov. & & $12 / 30 / 98$ & $12 / 30 / 98$ & 0.75 & $0 \%$ & & & & & & & & & & \\
\hline 19320 & Ceramic Tip Shos Evaluation & & 711496 & $11130 / 98$ & of & $25 \%$ & & & $\square$ & & $\nabla$ & & & & & \\
\hline 19320.1 & Complote Procurement, Ceramic Tip Shoes & - & $5 / 30 / 97$ & $5 / 30 / 97$ & 0.25 & $100 \%$ & & & & $\Delta$ & & & & & & \\
\hline 19320.2 & Complate ceramic Tip Shoo Evaluation & & i1/30/98 & $11130 / 98$ & 0.75 & $0 \%$ & $\ldots$ & & & & $\Delta$ & & & & & \\
\hline 19230 & Coramic Bushing / Ball Bearing & & $911 / 96$ & $1223 / 97$ & & $25 \%$ & $\cdots$ & & 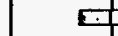 & & & & & & & \\
\hline 19330.1 & Complato Procurement, Bail / Ball Bearing & & $5 / 30 / 97$ & 53099 & 0.25 & t00\% & ............ & & & $\Delta$ & & & & & & \\
\hline 19330.2 & Complate, Cerramic Bushing / Ball Bearing & & $12 / 23 / 97$ & $12 / 23 / 97$ & 0.75 & $0 \%$ & & & & & & & & & & \\
\hline 19340 & Coramic Interstage Ring Seal Dev. & & $10 / 1 / 96$ & $6 / 30 / 99$ & of & $10 \%$ & 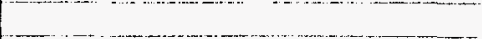 & & 网 & & & & & & & \\
\hline 19340.1 & Complele Solection, Ring Seal Malerial & & $122 / 96$ & 1212196 & 0.10 & $100 \%$ & ... & & 4 & & & & & & & \\
\hline 19340.2 & Complete Rub Testing - Ring Soal & & $12 / 30 / 97$ & $12 / 30 / 97$ & 0.25 & $0 \%$ & $\ldots$ & & & & & & & & & \\
\hline 19340.3 & Completo Ceramic Interslage Ring Seal Dav. & & $6 / 30 / 99$ & $6 / 30 / 99$ & 0.65 & $0 \%$ & - & & & & & $\Delta$ & & & & \\
\hline 19330 & Ceramic Blade Dev. & & 9114195 & 9/30/98 & & $10 \%$ & & & & & & & & & & \\
\hline 19350.1 & Complete Material Selection, Ceramic Blade Dov. & & $2 / 29 / 96$ & $2 / 29 / 96$ & 0.10 & $100 \%$ & & & $\mathbf{A}$ & & & & & & & \\
\hline 19350.2 & Complete, Atachment Test - Elade & & $130 / 98$ & $1 / 30 / 98$ & 0.25 & $0 \%$ & - & & & & & & & & & \\
\hline 19350.3 & Complete, Prool Test - Elade & & $730 / 98$ & $7 / 30198$ & 0.25 & $0 \%$ & $\ldots$ & & & & $\Delta$ & & & & & \\
\hline 19350.4 & Complete, Corramic Blade Dev. & & $9 / 30 / 98$ & $9230 / 98$ & 0.40 & $0 \%$ & $x_{1}$ & & & & $\Delta$ & & & & & \\
\hline 19360 & Ceramic Nozzle Dev. & & $1 / 2 / 96$ & $10 / 29 / 99$ & of & $10 \%$ & 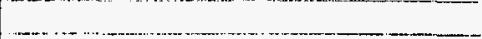 & & एका & & & & & & & \\
\hline 19360.1 & Completo Material Selection, Ceramic Nozzlo & & $6 / 27 / 97$ & 6,2797 & 0.10 & $100 \%$ & . & & & $\Delta$ & & & & & & \\
\hline 19360.2 & Complete, Procure Speciment - Ceramic Nozzle & & $6 / 30 / 98$ & $6 / 20098$ & 0.15 & $0 \%$ & ... & & & & $\Delta$ & & & & & \\
\hline 19360.3 & Complete, Simulation Testing - Ceramic Noz zle & & $1 / 30 / 99$ & $130 / 99$ & 025 & $0 \%$ & $\ldots \ldots \ldots$ & & & & & & & & & \\
\hline 19360.4 & Complete, Ceramic Nozzla Dev. & & $8 / 3199$ & 831199 & 0.25 & $0 \%$ & 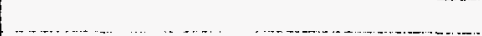 & & & & & $\Delta$ & & & & \\
\hline 19360.5 & Complete Topical Report Cerremic a Composito Materia & & $10 / 29 / 99$ & $10 / 29 / 99$ & 0.25 & $0 \%$ & 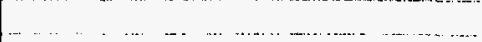 & & & & & $\Delta$ & & & & \\
\hline 19400 & Turbine Material Development & & $1 / 2196$ & $12 / 30 / 9$ & 0 & $25 \%$ & & & Proter & & & & & & & \\
\hline 19410 & Forged Disk Materials & & viz/96 & $1230 / 99$ & & $30 \%$ & $1-\ldots$ & & 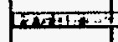 & $I$ & & & & & & \\
\hline 19410.1 & Complete, Prolotype Forged Disk & & 330197 & $3 / 301997^{\circ}$ & 0.10 & $100 \%$ & Design Into to Design & & & $\Delta$ & & & & & & \\
\hline 19410.2 & Doliver, Prelim Design Inlo to Design - Forged Disk & & $1 / 15 / 97$ & $11 / 15 / 97$ & 0.10 & $100 \%$ & & & & & & & & & & \\
\hline 19410.3 & Stort, Mechanical Propentios Tessis - Forged Disk & & $6 / 30 / 97$ & $6 / 30199$ & 0.10 & $100 \%$ & & & & $\underline{\Delta}$ & & & & & & \\
\hline 19410.4 & Complete, Scale Design - Forged Disk & & $2 / 28 / 98$ & 2228189 & 0.15 & $0 \%$ & (2) & & & & & & & & & \\
\hline 18410.5 & Complette Lile Predictions - Forged Disk & & $8 / 15 / 98$ & $8 / 15 / 98$ & 0.15 & $0 \%$ & & & & & $\Delta$ & & & & & \\
\hline 19410.6 & Complete, Engino Test - Forged Disk & & $12 / 15 / 98$ & $1215 / 98$ & 0.25 & $0 \%$ & & & & & $\Delta$ & & & & & \\
\hline 19810.7 & Complete, Topical Report, Disk Matorials Dev. & & $12 / 30 / 99$ & $12 / 30 / 99$ & 0.15 & $0 \%$ & & & & & & & & & & \\
\hline 19420 & Oual Alloy Disks & & $1 / 296$ & $1230 / 99$ & . & 186 & & & & & & & & & & \\
\hline 19420.1 & $\begin{array}{l}\text { Comptete, Alloy Cost Evaluation - Duan Alloy Diska } \\
\text { Completo, Engino Test - Dual Alloy Disk }\end{array}$ & & $\mid \begin{array}{l}12 / 15 / 97 \\
10 / 30999\end{array}$ & $12115 / 97$ & $\begin{array}{r}0.15 \\
0.25\end{array}$ & $\% \%$ & & & & & & & & & & $\cdots$ \\
\hline$\frac{19420.2}{19420.3}$ & $\begin{array}{l}\text { Complete, Engino Test - Dual Alloy Disk } \\
\text { Completo, Mechanical Propenties - Dual Alloy Disk }\end{array}$ & & $\mid \begin{array}{l}10 / 30 / 99 \\
11 / 30 / 96\end{array}$ & $\left|\begin{array}{l}1030 / 99 \\
11 / 30 / 98\end{array}\right|$ & $\begin{array}{r}0.25 \\
0.35\end{array}$ & $0 \%$ & 10/97 Design Info lo Dosign & & & & & $\Delta$ & & & & \\
\hline$\frac{19420.3}{19420.4}$ & 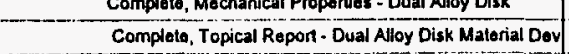 & & $12 / 30 / 99$ & $\mid \begin{array}{l}1 / 30 / 98 \\
12 / 30 / 99\end{array}$ & -0.25 & $\begin{array}{ll}0 \% \\
0 \% \\
0 \%\end{array}$ & & & & & $\Delta$ & & & & . & \\
\hline 19430 & CMSX-10 Material Dev & $\ldots$ & $1 / 2 / 96$ & 1223398 & {$[-0$} & $30 \%$ & $-1-1-1-1-1-1$ & & Exent & & & & & & & $\cdots-$ \\
\hline
\end{tabular}

Printed: 10/30/97

Page 15 
Advanced Turbine Systems

Phase 3 and 4

Filter: Total Program

\begin{tabular}{|c|c|c|c|c|c|c|c|c|c|c|c|c|c|c|c|c|}
\hline was & Task Name & Dwg / Part No & $\begin{array}{l}\text { Start } \\
\text { Date }\end{array}$ & $\begin{array}{l}\text { End } \\
\text { Date }\end{array}$ & Value & \begin{tabular}{|c|}
$\begin{array}{c}\text { Percent } \\
\text { Complete }\end{array}$ \\
\end{tabular} & Notes & 1995 & 1998 & 1997 & 1080 & 1990 & 2000 & 2001 & 2002 & 2003 \\
\hline 19930.1 & Complote, Casting Trials - CMSX-10 & & $7115 / 26$ & $7115 / 90$ & 0.15 & $100 \%$ & & & 4 & & & & & & & \\
\hline 194302 & Complota, Coating Evaluation - CMSX-10 & & $4 / 30197$ & 1/30/97 & 0.15 & $100 \%$ & & & & $\Delta$ & & & & & & \\
\hline 19430.3 & Complete, Mochanical Properties - CSMX-10 & & $5 / 30 / 98$ & $5 / 30198$ & 0.15 & $0 \%$ & & & & & $\Delta$ & & & & & \\
\hline 194304 & Complete, Engine Test - CSMx-10 & & $1015 / 90$ & $10 / 15 / 88$ & 025 & $0 \%$ & & & & & $\Delta$ & & & & & \\
\hline 19430.5 & Complete, Topical Report - CMSX-10 Material Dov. & & 1230198 & $12 / 30 / 98$ & 025 & $0 \%$ & & & & & & & & & & \\
\hline 194140 & Low CTE Matorlals & & indis & $12 / 23 / 98$ & & $20 \%$ & & & mace & & & & & & & \\
\hline 19440.1 & Complete. Forging Dev. - CTE & & $1 / 15 / 96$ & $1 / 15 / 96$ & 0.15 & $100 \%$ & & & s & & & & & & & \\
\hline 19440.2 & Complete. Prototype Demo (12) - CTE & & $8 / 25 / 97$ & $8 / 25 / 97$. & 0.05 & $100 \%$ & Changed to engine specific materials. & & & $\Delta$ & & & & & & \\
\hline 19440.3 & Complete, Heat Treal Optimization - CTE & & $12 / 30 / 97$ & $12 / 30 / 97$ & 0.15 & $0 \%$ & 12/96 Design Inlo lo Design & & & & $\Delta$ & & & & & \\
\hline 19440.4 & Complete, Prolotypo Demo (10) - CTE & & 1715/98 & ins/98 & 0.10 & $0 \%$ & Changed to engine specific materials. & & & & & & & & & \\
\hline 19440.5 & Complete, Mechanical Properties -CTE & & $6 / 20 / 98$ & $8 / 30 / 98$ & 0.15 & $0 \%$ & & & & & $\Delta$ & & & & & \\
\hline 19440.6 & Complete, Engine Test - CrE & & $100 / 30 / 9 a^{\circ}$ & i0/30/98 & 0.25 & $0 \%$ & & & & & $\Delta$ & & & & & \\
\hline 19440.7 & Complete. Topical Raport - Low CTE Materials Dovelop & & $12 / 30 / 98$ & $12 / 30 / 98$ & 0.15 & $0 \%$ & & & & & 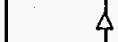 & & & & & \\
\hline 19450 & Advanced Casting Tochniques (AdCsi) & & $11296^{\circ}$ & $12 / 30 / 99$ & 9 & $30 \%$ & & & 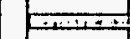 & & & & & & & \\
\hline 19450.1 & Complete, PMC Alloy Selection - AdCst & & $15 / 96$ & $15 / 96$ & 0 id & $100 \%$ & & & $\Delta$ & & & & & & & \\
\hline 19450.2 & Complete, Spraycast Alloy Sellection - AdCst & & $5 / 1 / 96$ & $5 / 1 / 196$ & 0.10 & $100 \%$ & : & & $\Delta$ & & & & & & & \\
\hline 19450.3 & Complete, Perm Mold Casting Process - AdCst & & $10 / 31 / 96$ & $10 / 31 / 96$ & 0.15 & $100 \%$ & 11/96 Design Info to Design & & $\Delta$ & & & & & & & \\
\hline 19450.4 & Complete Oplimize Spray Cast Process - AdCst & & $1 / 3497$ & $1 / 34 / 97$ & 0.05 & $100 \%$ & & & & $\Delta$ & & & & & & \\
\hline 19450.48 & Comploto, Oplimization HT / HIP for Haynes 242 - AdCst & & $3 / 1 / 97$ & $3 / 1197$ & 0.10 & $100 \%$ & & & & $\Delta$ & & & & & & \\
\hline 19450.5 & Complete, Mechanical Properties - AdCst & & $6 / 30 / 98$ & $6 / 30 / 98$ & 0.15 & $0 \%$ & 2987 Spraycast Design Inca ta Design & & & & $\Delta$ & & & & & \\
\hline 19450.6 & Completa, Engine Test - AdCst & & $10 / 30 / 99$ & $10 / 30 / 99$ & 0.20 & $0 \%$ & & & & & & $\Delta$ & & & & \\
\hline 19450.7 & Complete, Topical Repon - Adv. Casting Tochniques & & $12 / 30 / 99$ & $12 / 30 / 99$ & 0.15 & $0 \%$ & & & & & & & & & & \\
\hline 19460 & NDE / LiHO Prodiction (NDE) & (-) & $1 / 2 / 96$ & $12123 / 98$ & & $15 \%$ & - & & बहता & & & & & & & \\
\hline 19460.1 & Complete, Program Plans - NDE & & $4 / 30 / 96$ & 40 & 0.15 & $100 \%$ & & & $\Delta$ & & & & & & & \\
\hline 19460.2 & Complete, Tochnique Demos - NDE & & $6 / 1198$ & $6 / 198$ & 0.20 & $0 \%$ & Replanned one year out on 6-1.97 & & & & $\Delta$ & & & & & \\
\hline 19460.3 & Completo Dev. Inspection Techniques - NDE & & $8 / 31 / 98$ & $8 / 31 / 98$ & 0.25 & $0 \%$ & - & & & & $\Delta$ & & & & & \\
\hline 194604 & Complete, Analyzo Components - NDE & & $12 / 24 / 98$ & $12 / 24 / 98$ & 0.15 & $0 \%$ & & & & & & & & & & \\
\hline 19460.5 & Completo. NOE Lite Predictions & & $12 / 24 / 98$ & $12124 / 90$ & 0.25 & $0 \%$ & 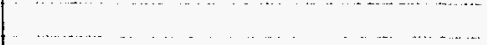 & & & & & & & & & \\
\hline 19500 & Recuperator Malerial Development & & 9/14/95 & $12130 / 99$ & & $25 \%$ & & & & & & & & & & \\
\hline 19500.1 & Complete, Ptan For Malerial Selection - Recup & & $5 / 3 / 96$ & $5 / 3 / 96$ & 0.10 & $100 \%$ & ........... & & $\Delta$ & & & & & & & \\
\hline $19500 . \overline{2}$ & Complete Fabrication Trials - Recup & & $3 / 1 / 97$ & $3 / 1 / 197$ & 0.15 & $100 \%$ & 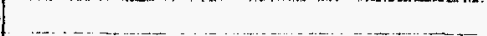 & & & $\Delta$ & & & & & & \\
\hline 19500.3 & Complete, Cost Model - Recup & & 1211197 & $12 / 15 / 97$ & 0.10 & $0 \%$ & - & & & & & & & & & \\
\hline 19500.4 & Complete, Mochanicat Properies - Rocup & & $12 / 15 / 98$ & $12115 / 98$ & 0.15 & $0 \%$ & 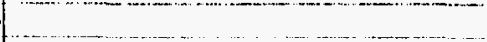 & & & & & & & & & \\
\hline 19500.5 & Complete, Engine Test - Recup & & $11 / 30 / 99$ & $11130 / 99$ & 0.25 & $0 \%$ & . & & & & & & & & & \\
\hline 19500.6 & Complate, Topical Roport, Recuperator Malenial Dev. & & $12 / 30 / 99$ & $12 / 30 / 99$ & 0.25 & $0 \%$ & 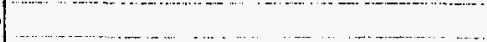 & & & & & & & & & \\
\hline 19600 & Variable Area Nozzle (VAN) (EMort on Hold) & & ivi/98 & $9 / 23 / 98$ & of & $0 \%$ & No Current Neod for VAN & & & & ב & & & & & \\
\hline 19700 & Advanced Coating Systems & & 9/14/95 & $12 / 17 / 9$ & 0 & $32 \%$ & & & & & & & & & & \\
\hline 19710 & Themal Barrier Coalings (TBC) & & 911495 & 12217198 & of & $30 \%$ & 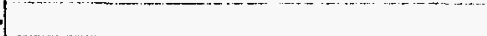 & & aI & & & & & & & \\
\hline 19710.1 & Complete. System Evaluation - TBC & & 773196 & 733196 & 0.10 & $100 \%$ & $8 / 96$ Design Info lo Dosign & & $\Delta$ & & & & & & & \\
\hline 197102 & Complete, Screoning Tessis - TBC & & $6 / 30 / 97$ & 6/30197 & 0.10 & $100 \%$ & 7997 Design into to Design & & & $\Delta$ & & & & & & \\
\hline 19710.3 & Complete, Mars Field Tost - TBC & & $6 / 30 / 97$ & 6/30/97 & 0.10 & $100 \%$ & Design Info to Design & & & $\bar{\Delta}$ & & & & & & \\
\hline 197104 & Complate, AIS Component Evaluation - TBC & & $10 / 31 / 97$ & $10 / 31 / 97$ & 0.10 & $0 \%$ & & & & & & & & & & \\
\hline 19710.5 & Complete, Lite Pridictions - TBC & & $12 / 3197$ & $12 / 31 / 197$ & 0.10 & $0 \%$ & 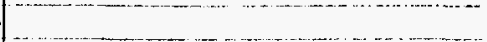 & & & & & & & & & \\
\hline 19710.6 & Complete, ATS Component Coatings - $T \mathrm{BC}$ & & $12 / 3197$ & $12 / 31 / 97$ & 0.10 & $\%$ & & & & & & & & & & \\
\hline 19710.7 & Complate Assy, Engino Harctware - TBC & & $3 / 31 / 98$ & $3 / 31 / 98$ & 0.10 & $0 \%$ & 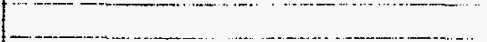 & & & & $\Delta$ & & & & & \\
\hline 19710.8 & Complete Test, Engine Hardware - I BC & & 11130/98 & $11 / 30 / 98$ & 0.15 & $0 \%$ & & & & & $\Delta$ & & & & & \\
\hline 19710.0 & Complete Topical Repor, Adv Coating Systems Dov. & & 1211798 & $12 / 17 / 98$ & 0.15 & $0 \%$ & 1 & & & & & & & & & \\
\hline 19720 & Chemelal Vapor Depositton (CVD) & & $1 / 2296$ & $12123 / 97$ & of & \$20\% & (a) & & m & & & & & & & \\
\hline 19720.1 & Complate, Sample Coalings - CVO & & $8 / 30 / 96$ & $8 / 30 / 96$ & 0.25 & $100 \%$ & $-1+1-1+-1$ & & $\boldsymbol{\Delta}$ & & & & & & & \\
\hline
\end{tabular}

Printed: 10/30/97

Page 16 
Advanced Turbine Systems

Phase 3 and 4

Filter: Total Program

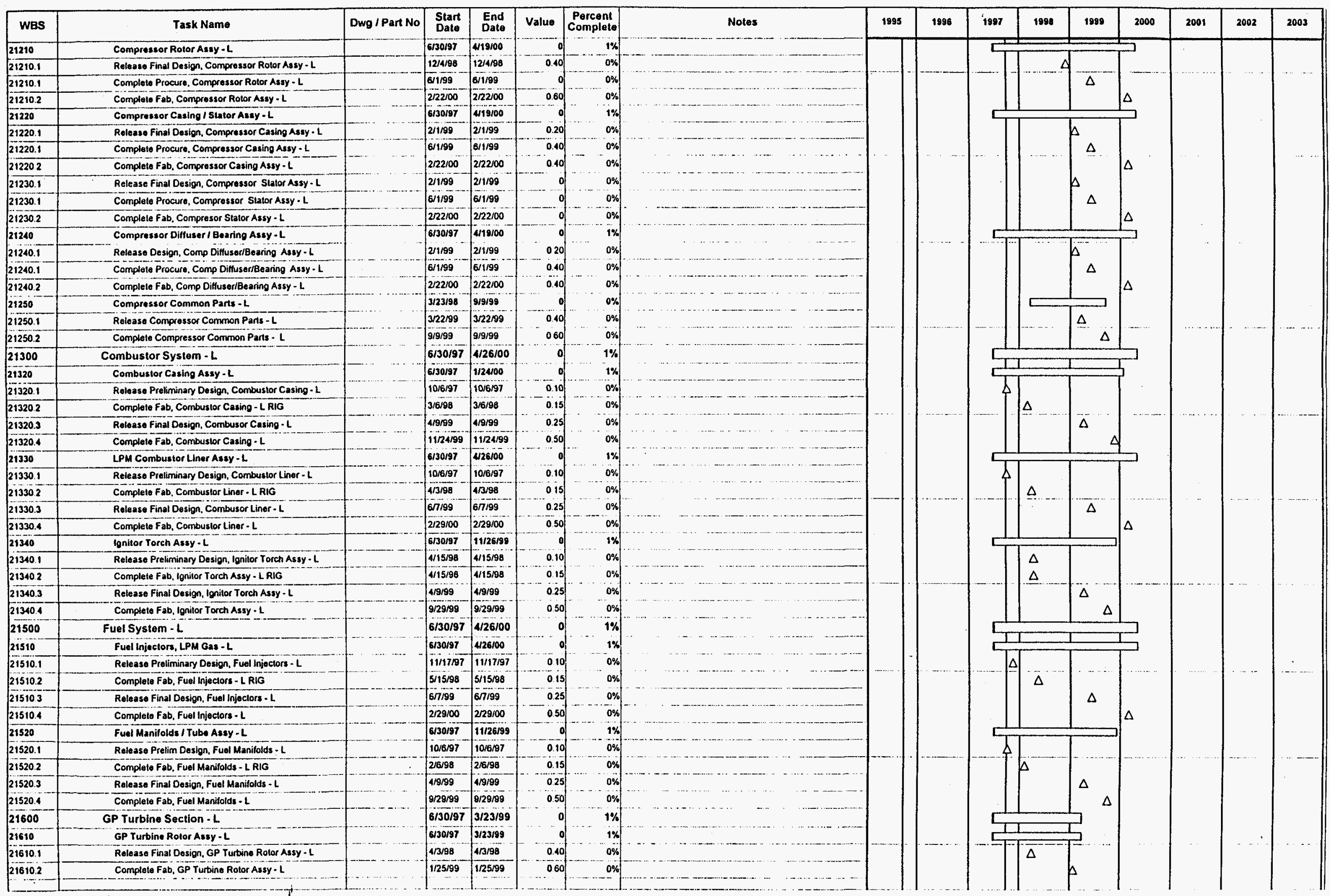

Printed: 10/30/97

Page 18 
Advanced Turbine Systems

Phase 3 and 4

Filter: Total Program

\begin{tabular}{|c|c|c|c|c|c|c|c|c|c|c|c|c|c|c|c|c|}
\hline WBS & Task Name & Dwg / Part No & $\begin{array}{l}\text { Start } \\
\text { Date }\end{array}$ & $\begin{array}{l}\text { End } \\
\text { Dato }\end{array}$ & Value & \begin{tabular}{|c|}
$\begin{array}{c}\text { Percent } \\
\text { Complete }\end{array}$ \\
\end{tabular} & Notes & 1995 & 1998 & 1997 & 1998 & 1999 & 2000 & 2001 & 2002 & 2003 \\
\hline 19720.2 & Complete, Process Assesssment - CVO & & $1 / 15 / 97$ & $1115 / 9$ & 0.15 & $100 \%$ & & & & 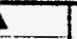 & & & & & & \\
\hline 19720.2 & Complete, Optimize Processs - CVD & & $12 / 30 / 97$ & $12 / 30 / 97$ & 0.20 & $0 \% /$ & Priboritzation & & & & & & & & & \\
\hline 19720.3 & Complete, Chamical Vapor Deposition Tests - CVO & & $12 / 30 / 97$ & 12130197 & 0.40 & $\%$ & 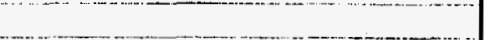 & & & & & & & & & \\
\hline 19730 & Alloy Coating Systoms (Coat) & & $51+196$ & 5/29189 & of & $50 \%$ & & & -1 & & & & & & & \\
\hline 19730.1 & Complete. Extermal Coatability - Cost & & $7115 / 97$ & $7 / 15 / 97$ & 0.25 & $100 \%$ & & & & $\Delta$ & & & & & & \\
\hline 19730.2 & Complete, Intemal Coatability - Coat & & 8201907 & 820197 & 0.25 & $100 \%$ & & & & & & & & & & \\
\hline 19730.3 & Complate, Alloy Conting Systems & & $5 / 31 / 98$ & $5 / 31 / 9 \theta^{\circ}$ & 0.50 & $0 \%$ & & & & & $\Delta$ & & & & & \\
\hline 19000 & Advanced Diffuser Development (ADD) & & M1495 & $12 / 23 / 97$ & 0 & $75 \%$ & & & & & & & & & & \\
\hline 19800.1 & Complete, Design Annular Model - ADD & & $1 / 30 / 96$ & $430 / 96$ & 0.10 & $100 \%$ & & & $\Delta$ & & & & & & & \\
\hline 198002 & Complete, Test Annular Model - ADD & & $9 / 30 / 96$ & $9 / 30 / 96$ & 020 & $100 \%$ & $\ldots$ & & $\Delta$ & & & & & & & \\
\hline 19800.3 & Stan, Oesign Engino Diffuser - ADD & & $9 / 30 / 96$ & 9/30/96 & 0.20 & $100 \%$ & & & $\Delta$ & & & & & & & \\
\hline 19800.4 & Completo, Test Engine Dilluser - ADD & & $5 / 31 / 97$ & $5 / 31 / 97$ & 025 & $100 \%$ & Tesl delerred lo S Engine duo to test engine availibility & & & $\Delta$ & & & & & & \\
\hline 19800.5 & Complete, Topical Report, Advanced Difuser & & $12 / 30 / 97$ & $12 / 30 / 97$ & 0.25 & $0 \%$ & & & & & & & & & & \\
\hline 19900 & Advanted Man-Machine Interface & & N15/96 & 4/21/98 & of & $15 \%$ & . & & $\square$ & & ] & & & & & \\
\hline 19900.1 & Complete, Analyze Dala - MMI & & 5416,997 & $5 / 16 / 97$ & 0.15 & $100 \%$ & & & & $\Delta$ & & & ' & & & \\
\hline 19900.2 & Complete, Test-MMI & & 121189 & in21/98 & 0.20 & $0 \%$ & & & & & 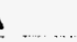 & & & & & \\
\hline 19900.3 & Complater Standardize - MMI & & $3 / 20198$ & 320199 & 0.25 & $\%$ & $\ldots \ldots \ldots$. & & & & $\Delta$ & & & & & \\
\hline 19900.4 & Complote, Topical Report - MMI & & 421198 & $1 / 21 / 98$ & 0.40 & $0 \%$ & - & & & & $\Delta$ & & & & & \\
\hline 20000 & Advanced Turbine Systom $-L$ & & $1 / 2 / 96$ & $5 / 1 / 1 / 2$ & 0] & $8 \%$ & $\ldots$ & & 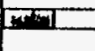 & & & & & & & \\
\hline 20100 & Technical Coordination $-L$ & & $5 \leqslant 197$ & sil1102 & 0 & $6 \%$ & $\ldots$ & & & & & & & & & \\
\hline 20200 & Engina Definition $-\mathrm{L}$ & & 12296 & 1331987. & of & 100\% & 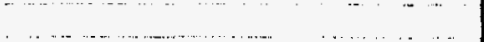 & & EDis & 年 & & & & & & \\
\hline 20200.1 & Complete ist Dran, Marketing Regunt Spec - $L$ & & $5 / 10 / 98$ & $5 / 10096$ & 0.10 & $100 \%$ & & & $\Delta$ & & & & & & & \\
\hline 20200.2 & Complete. 181 Engine Cross-section Dug $-L$ & & $6 / 1196$ & $6 / 1 / 96$ & 0.10 & $100 \%$ & & & $\Delta$ & & & & & & & \\
\hline 20200.3 & Complete ist Draft, Product Spoc - $\mathrm{L}$ & & $6 / 25 / 96$ & $6 / 25 / 96$ & $0.10^{\circ}$ & $100 \%$ & 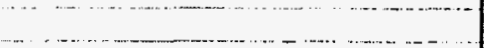 & & $\Delta$ & & & & & & & \\
\hline 20200.4 & Complete ist Dratt, Business Plan Reviow - $L$ & & 711096 & $7110 / 96$ & 0.10 & $100 \%$ & $\ldots$ & & $\Delta$ & & & & & & & \\
\hline 20200.5 & Revised Markel Reqmi Spec - $L$ & & $11 / 1198$ & (11/1/196 & 0.10 & $100 \%$ & $\ldots$ & & $\Delta$ & & & & & & & \\
\hline 20200.6 & Complete Dran, Business Ptan - L & & $12 / 10 / 96$ & $12 / 10 / 96$ & 0.05 & $100 \%$ & 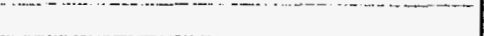 & & & & & & & & & \\
\hline 20200.7 & Present, Business Plan For PC Approval - $L$ & & $121 / 97$ & $1 / 2199$ & 0.10 & $100 \%$ & - & & & $\Delta$ & & & & & & \\
\hline 202000. & Finalize, Business Plan - 1 & - & 228397 & 228897 & 0.15 & $100 \%$ & (n) & & & $\Delta$ & & & & & & \\
\hline 202009 & Approval, Business Plan - L & 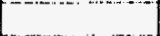 & $3 / 31 / 97$ & $3 / 31 / 97$ & 0.10 & $100 \%$ & -1 & & & $\Delta$ & & & & & & \\
\hline 202009 & Complete, Concept Design - I & & $8 / 3197$ & $8 / 31 / 97$ & 0.10 & $100 \%$ & & & & & & & & & & \\
\hline 20300 & Package Definition- $L$ & & 71199 & 10/31197 & & $1 \%$ & $\ldots$ & & & & & & & & & \\
\hline 20300.1 & Complete, System Design Review - L & & $10 / 31 / 97$ & $10 / 3197$ & 1.00 & $0 \%$ ] & - & & & & & & & & & \\
\hline 21000 & Engine Assembly $-L$ & & $6 / 30 / 97$ & Bition. & & $1 \%$ & & & & & & & & & & \\
\hline 21010 & Engine Design Support - L & & 731197 & $3 / 30101$ & & $1 \%$ & 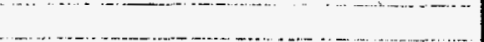 & & & & & & & & & \\
\hline 21020 & Engine Build $-L$ & & $215 / 99$ & stues & of & $0 \%$ ] & $\ldots$ & & & & & 5 & & $\Longrightarrow$ & & \\
\hline 210101 & Complote, Engino Assembly Drawings - $L$ & & $0 / 29 / 99$ & $9 / 29 / 99$ & 0.25 & $0 \%$ ? & $-x^{\prime}$ & & & & & $\bar{\Delta}$ & & & & \\
\hline 21010.2 & Cash, Engine $\$ 1-1$ & & $11 / 1 / 199$ & $111 / 199$ & 0.40 & $0 \%$ & $\ldots$ & & & & & $\Delta$ & & & & \\
\hline 21010.3 & Cash, Engine $* 2-L$ & & $2111 / 100$ & 221100 & of & $0 \%$ & & & & & & & $\Delta$ & & & \\
\hline 210104 & Complele Build, Engine $* 3 . \mathrm{L}$ & & $1 / 3 \% 01$ & $1 / 31 / 01$ & 0 & $0 \%$ & . & & & & & & & & & \\
\hline 21010.5 & Complete Rebuild, DOE Demo Engine $\| 1-L$ & & $8 / 1 / 01$ & $8 / 1 / 101$ & 0.35 & $0 \%$ & & & & & & & & $\Delta$ & & \\
\hline 21100 & Air Injet System - L & & $6 / 30 / 97$ & $3 / 8100$ & 0 & $1 \%$ & & & & ¿ & & & & & & \\
\hline 211110 & Inlat Collecior $-L$ & & $6 / 630197$ & (8/26/99 & of & $1 \%$ & - & & & & & & & & & \\
\hline 21110.1 & Release Final Dosign Inlet Air Assembly - $L$ & & $5 / 1 / 198$ & $5 / 1 / 98$ & 0.40 & $0 \%$ & $\ldots \ldots$ & & & & $\Delta$ & & & & & \\
\hline 21110.2 & Complete Fab, Inlet Air Assembly - $L$ & & 6212999 & $60 / 29 / 99$ & 0.60 & $0 \%$ & $\ldots \ldots \ldots \ldots$ & & & & & $\Delta$ & & & & \\
\hline 21120 & Inibt Air Assembly $-L$ & & $8 / 30197$ & $38 / 00$ & & $1 \%$ & 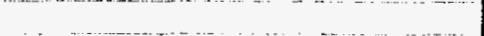 & & & & & & & & & \\
\hline 21120.1 & Rolaase Final Design, Intel Air Assembly - $L$ & & $101 / 98$ & 10it198 & 0.40 & $0 \%$ & $\cdots$ & & & & $\Delta$ & & & & & \\
\hline 21120.2 & Complete Fab, Inlel Air Assembly - $\mathrm{L}$ & - & $1 / 11100$ & 1111100 & 0.60 & $0 \%$ & & & & & & & & & & \\
\hline 21200 & Compressor Assembly - L & & $6 / 30 / 97$ & $4 / 19 / 00$ & & $1 \%$ & $\ldots$ & & & & & & & & & \\
\hline
\end{tabular}

Printed: 10/30/97

Page 17 
Advanced Turbine Systems

Phase 3 and 4

Filter: Total Program

\begin{tabular}{|c|c|c|c|c|c|c|c|c|c|c|c|c|c|c|c|c|}
\hline WBS & Task Name & Dwg / Part No & $\begin{array}{l}\text { Start } \\
\text { Date }\end{array}$ & $\begin{array}{c}\text { End } \\
\text { Date }\end{array}$ & Value & $\begin{array}{c}\text { Percent } \\
\text { Complete }\end{array}$ & Notes & 1995 & 1996 & 1897 & 1998 & 1999 & 2000 & 2001 & 2002 & 2003 \\
\hline 21620 & Turbine Casing Assy - L & & 6130/97 & $9 / 1099$ & 9. & $1 \%$ & & & & & $コ$ & & & & & \\
\hline 21620.1 & Rolease Final Design, Turtine Casing Assy - L & & $123 / 98$ & $1 / 23 / 98$ & 0.40 & $0 \%$ & & & & & & & & & & \\
\hline 21620.2 & Complete Fab. Turtine Casing Assy - L & & 711498 & 2114498 & 0.60 & $0 \%$ & & & & & $\Delta$ & & & & & \\
\hline 21630 & GP Turbine Nozxto Assy - L & & $6 / 30197$ & $8 / 10 / 98$ & of. & $1 \%$ & & & & & $\square$ & & & & & \\
\hline 216301 & Rolease Final Design, GP Turbine Nozzle Assy - L & & $1 / 23 / 98$ & $123 / 88$ & $0.40 \%$. & $.0 \%$. & 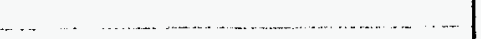 & & & & & & & & & \\
\hline 21630.2 & Complele $F$ ab, GP Tultina Nozzlo Assy - $\mathrm{L}$ & & 711498 & 711448 & 0.60 & $0 \%$ & - & & & & $\Delta$ & & & & & \\
\hline 21640 & GP Common Parts $-L$ & & 6/30/98 & $1125 / 99$ & & $1 \%$ & $\ldots \ldots \ldots \ldots$ & & & & 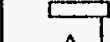 & & & & & \\
\hline 21640.1 & Roiease, GP Common Parls - $L$ & & $9 / 11 / 98$ & $9 / 11 / 98$ & 0.40 & $0 \%$ & $\ldots \ldots \ldots$ & & $\cdots$ & & $\Delta$ & & & & & \\
\hline $21640.2 \ldots$ & Complete. GP Common Pans - $L$ & & $1 / 25 / 99$ & $1 / 25 / 99$ & 0.60 & $0 \%$ & $\ldots \ldots \ldots$ & & & & & e & & & & \\
\hline 21700 & Power Turbine Section $-L$ & & $6 / 30 / 97$ & $5 / 4 / 99$ & & $1 \%$ & $\ldots \ldots \ldots$ & & & & I & & & & & \\
\hline 21710 & Power Turbine Rotor Assy - L & & $6 / 30 / 97$ & 514199 & & 18 & . . . n . & & & & & & & & & \\
\hline $21710 . !$ & Release Final Design, Powar Turtine Rolor Assy $\cdot \ell$ & & $5 / 1 / 98$ & $5 / 1 / 98$ & 0.40 & $0 \%$ & $\ldots$ & & & & $\Delta$ & & & & & \\
\hline 217102 & Complete Fab, Power Turbine Rolor Assy $-L$ & & $3 / 8 / 99$ & $3 / 8 / 99$ & 060 & $0 \%$ & $\cdots \cdots$ & & & & & $\Delta$ & & & & \\
\hline 21720 & PI Nozzle Assy -L & & $6 / 30 / 97$ & $7,29 / 98$ & o & $1 \%$ & 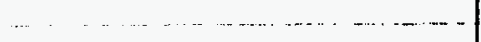 & & & & & & & & & \\
\hline 21720.1 & Release Final Design, PT Nozzle Assy - 1 & & $12 / 3 / 97$ & $12 / 3 / 97$ & & $0 \%$ & $\ldots \quad \ldots$ & & & 4 & & & & & & \\
\hline 217202 & Complete Fab, PT Nozzle Assy - L & & $6 / 1 / 98$ & $6 / 1 / 98$ & & $0 \%$ & $\ldots \ldots \ldots$ & & & & $\Delta$ & & & & & \\
\hline 21730 & PT Common Pans - L & & $6 / 1 / 98$ & $1 / 25 / 99$ & & $0 \%$ & $\ldots \ldots \ldots \ldots$ & & & & 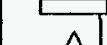 & & & & & \\
\hline 21730.8 & Rolease, PT Common Pans - $L$ & & 9rargi & $9 / 30 / 98$ & $\begin{array}{l}0.40 \\
0.60\end{array}$ & $\begin{array}{ll}0 \% \\
0 \%\end{array}$ & 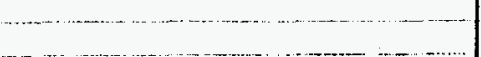 & & & & $\Delta$ & & & & & \\
\hline 21730.2 & Complete, PT Common Pans - $\mathrm{L}$ & 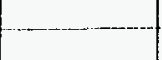 & $1 / 25 / 99$ & $125 / 99$ & & $1 \%$ & - & & & & & p & & & & \\
\hline 21800 & Exhaust Section - L & & $6 / 30 / 97$ & $5 / 17 / 99$ & & \% & $-\cdots+\cdots$ & & & 1. & 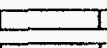 & & & & & \\
\hline 21010 & Exhaust Diffuser / Bearing Alsy $-L$ & & 630097 & 5317199 & 0.40 & $0 \%$ & ( & & & & $\Delta$ & & & & & \\
\hline 21810.1 & Release Final Design, Exhausl Diffuser / Bearing Assy - & & $4 / 15 / 98$ & $\begin{array}{l}4 / 15 / 98 \\
3 / 20 / 99 \\
3 / 29\end{array}$ & $\begin{array}{l}0.40 \\
0.60\end{array}$ & $0 \%$ & (1) & & & & & $\Delta$ & & & & \\
\hline 21810.2 & $\begin{array}{l}\text { Completele Fab, Exhaust Diffuser / Bearing Assy - L } \\
\text { Exhzust Colloctor Assy - L }\end{array}$ & & $\left|\begin{array}{ll}3 / 20 / 99 \\
6 / 30197\end{array}\right|$ & $\frac{3 / 20 / 99}{3 / 23 / 99}$ & & $i \%$ & . & & & & & & & & & \\
\hline 21820 & & & $16 / 30197$ & (1/15/15/97 & & $0 \%$ & $\ldots$ & & & $\Delta$ & & & & & & \\
\hline 21820.1 & $\begin{array}{l}\text { Release Final Design, Exhausi Colloctor Assy }-L \\
\text { Complete Fab, Exhaust Collector Assy }-L\end{array}$ & & 10 & $1 / 5 / 99$ & & $0 \%$ & 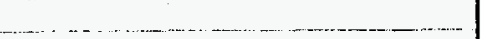 & & & & & $\vec{T}$ & & & & \\
\hline 21820.2 & $\begin{array}{l}\text { Complete Fab, Exhaust Collector Assy }-L \\
\text { Engine Common Parts } L\end{array}$ & & $5 / 1 / 98$ & $3 / 10 / 99$ & & $0 \%$ & 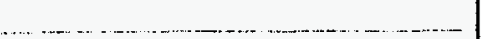 & & & & & 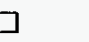 & & & & \\
\hline 21900 & $\begin{array}{l}\text { Engine Common Parts }-L \\
\text { Engine Common Parts }-L\end{array}$ & & 511198 & 3110199 & & $0 \%$. & & & & & $=-1$ & & & & & \\
\hline 21900 & $\begin{array}{l}\text { Engine Common Parts }-L \\
\text { Releaso Final Design, Bleed Valvo \& Duct Assy - } L\end{array}$ & & $10 / 15 / 98$ & 10/15/98 & 0.40 & $0 \%$ & $\ldots \ldots \ldots \ldots$ & & & & $\bar{\Delta}$ & & & & & \\
\hline 21910.1 & Compiete Fab, Bloed Valve \& Duct Assy - L & & $3 / 10 / 99$ & $3 / 10 / 99$ & 0.60 & $0 \%$ & 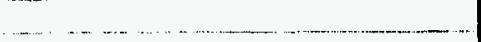 & & & & & $\Delta$ & & & & \\
\hline 21920.1 & Rele ase Design, Engina Assemly Hardwaro - $\mathrm{L}$ & & $10 / 15 / 98$ & $10 / 15 / 98$ & 0.40 & $0 \%$ & (1) & & & & $\Delta$ & & & & & \\
\hline 21920.2 & Complete Fab, Engine Assembly Hardware - $L$ & & $3 / 10 / 99$ & $3 / 10199$ & 0.60 & $0 \%$ & 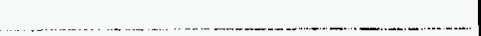 & & & & & $\Delta$ & & & & \\
\hline 22000 & Package Systems $-\mathbf{L}$ & & 9/2/97 & $2 / 14 / 02$ & & $0 \%$ & $\ldots \ldots \ldots$ & & & & & & & & & \\
\hline 22100 & Package Mechanical Systems - L & & $9 / 2 / 97$ & $3 / 9 / 01$ & & $0 \%$ & $\ldots \ldots \ldots$ & & & $\square$ & & & & & & \\
\hline 22110 & Generator $-L$ & & $92 / 297$ & 111000 & & $1 \%$ & & & & & & & & & & \\
\hline 22110.1 & Releaso, Generator Spec. - L & & 104497 & $10 / 497$ & 0.10 & $0 \%$ & 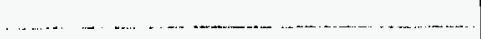 & & & $\Delta$ & & & & & & \\
\hline 22110.2 & Release, Generator Procurement Drawing $-L$ & & 10/18/97 & $10 / 18 / 97$ & 0.15 & $0 \%$ & $-1-2-1-1$ & & & $B$ & & & & & & \\
\hline 22110.3 & Review, Supplier Generalor Documentation - L & & $12 / 21 / 97$ & $12 / 21 / 97$ & 0.25 & $0 \%$ & 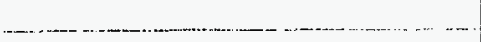 & & & & & & & & & \\
\hline 22110.4 & Procure, Generator - $\mathrm{L}$ & & 1110000 & $1 / 10 \% 0$ & 0.50 & $0 \%$ & (a) & & & & & & & & & \\
\hline 22120 & Reduction Geartox $-L$ & & 9/2/197. & 1117100 & of & 1\% & & & & & & & & & & \\
\hline 22120.1 & Release, Reduction Gearbox Spec. - L & & $10 / 15 / 97$ & $50 / 15 / 97$ & 0.10 & $0 \%$ & & & & t & & & & & & \\
\hline 221202 & Releaso, Reduction Gearbox Procurement Drawing - $\mathrm{L}$ & & $11 / 23 / 97$ & $11 / 23 / 97$ & 0.15 & $0 \%$. & & & & $\Delta$ & & & & & & \\
\hline 22120.3 & Review, Gearbox Supplier Documentation - $L$ & & $3 / 1998$ & $3 / 19 / 98$ & 0.25 & $0 \%$ & & & & & $\Delta$ & & & & & \\
\hline 22120.4 & Procure, Reduction Geartox - $L$ & & 13171700 & 1117700 & 0.50 & $0 \%$ & & & & & & & & & & \\
\hline 22130 & Electrical Conduit / J-Boxes - $L$ & & $66 / 3998$ & 14400 & o) & $0 \%$ & $-1,-1$ & & & & - & & & & & \\
\hline 22130.1 & Release, Electrical Conduit IJ-boxes - $-L$ & & $92 / 288$ & $9 / 2 / 98$ & 0.40 & $0 \%$ & 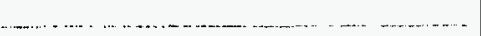 & & & & $\Delta$ & & & & & \\
\hline 22130.2 & Procuro Electrical System Components -1 & & 114100 & 14400 & 060 & $0 \%$ & & & & & & & & & & \\
\hline 22140 & Package I Generator Framo - L & & $3 / 10 / 39$ & 1127100 & & $\%$ & & & - & & $=$ & & & & & \\
\hline 22140.1 & Release, Final Design, Packago Frame $-L$ & & $115 / 98$ & $\mid \begin{array}{l}11 / 5 / 98 \\
-3\end{array}$ & $\quad 0.20$ & $0 \%$ & 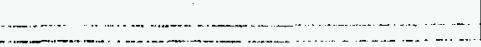 & & & & & & & & & \\
\hline
\end{tabular}

Printed: 10/30/97

Page 19 
Phase 3 and 4

Filter: Total Program

\begin{tabular}{|c|c|c|c|c|c|c|c|c|c|c|c|c|c|c|c|c|}
\hline WBS & Task Name & Dwg / Part No & $\begin{array}{l}\text { Start } \\
\text { Date }\end{array}$ & $\begin{array}{l}\text { End } \\
\text { Dalo }\end{array}$ & Value & $\mid \begin{array}{c}\text { Percent } \\
\text { Complete }\end{array}$ & Notes & 1995 & 1996 & 1897 & 1998 & 1998 & 2000 & 2001 & 2002 & 2003 \\
\hline 27200.2 & On Sitw - Balanco of Plant - $L$ & & $12 / 14 / 01$ & $12 / 14 / 01$ & 0.60 & $0 \%$ & & & & & & & & & & \\
\hline 28000 & Tooling / F ixtures - $L$ & & $3 / 25 / 98$ & $5 / 17100$ & of & $0 \%$ & & & & & & & ] & & & \\
\hline 23100 & Cold Section Tooling $\cdot L$ & & $3 / 25198$ & 5117100 & & a* & & & & & & & & & & \\
\hline 28200 & Hot Section Tooling $-L^{-}$ & & $32325 / 98$ & $5 / 177100$ & & $0 \%$ & $\ldots$ & & & & 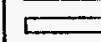 & & & & & \\
\hline $20300^{-1}$ & Rotors Tooling $-L$ & & $325 / 98$ & 51171700 & & $0 \%$ & $\ldots$ & & & & 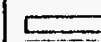 & 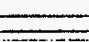 & & & & \\
\hline 28500 & Engine Assy Toolling $\cdot L$ & & $3 / 23 / 98$ & $3117100^{\circ}$ & & $0 \%$ & $\ldots$ & & & & 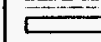 & & & & & \\
\hline 28600 & Production Tost Toolling $-L$ & & $429 / 86$ & $4119 / 99$ & & $0 \%$ & 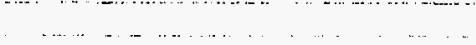 & & & & $\square$ & & & & & \\
\hline 28700 & Supenior Gear Tooling -L & & $3 / 25 / 98$ & $3 / 177100$ & & $0 \%$ & $\ldots \ldots$ & & & & $\square$ & & & & & \\
\hline 30000 & Product Support & & 9/14/95 & $12 / 5 / 02$ & & $2 \%$ & $\ldots \ldots \ldots$ & & & & & & & & & \\
\hline 30100 & Customer Services Coordination - $\mathbf{s}$ & & 9/14195 & $\mid 5 / 21 / 01$ & & $33 \%$ & 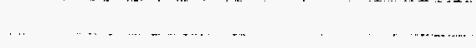 & & Tancerse & $\sin$ & & & & 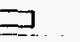 & & \\
\hline 30100 & Customer Senices Coordination - $L$ & & 9:1/97. & $12 / 5102$ & & $1 \%$ & $\ldots$ & & & & & & & & & \\
\hline 31000 & Product Support - S & & $3 / 1 / 96$ & $5 / 21 / 01$ & & $0 \%$ & $\ldots \ldots \ldots \ldots$ & & & & & & & & & \\
\hline 31100 & Product Support Planning - $S$ & & $3 / 1 / 96$ & $8 / 21 / 01$ & & $0 \%$ & $\ldots$ & & & & & & & & & \\
\hline 31110 & Product Support Plan -s & & $3 / 1 / 96$ & 71197 & & $1 \%$ & $-\cdots$ & & $\sqsubset$ & & & & & & & \\
\hline $3+110.1$ & Complete Product Support Plan - S & & $\mid 11 / 15 / 97$ & $111 / 15 / 97$ & & $0 \%$ & $\ldots \ldots$ & & & & & & & & & \\
\hline 31120 & Roc. Spare Parts List (RSPL).S & & $31 / 96$ & $\mid 415 / 97$ & & 1*: & $\ldots \ldots$ & & & & & & & & & \\
\hline 31120.1 & Complete, Spare Panst List - S & & $11115 / 97$ & $\mid 11 / 5 / 97$ & 100 & $0 \%$ & .................... & & & & & & & & & \\
\hline 31130 & Customer Senceas Provisioning Plan -S & & $3 / 196$ & 81197 & & $1 \%$ & (n............... & & & & & & & & & \\
\hline 31130.1 & Complete Customer Support Provision Plan - $\mathrm{s}$ & & i1115/97 & $11 / 15 / 97$ & 1.00 & $0 \%$ & - & & & & & & & & & \\
\hline 31140 & Senvice Support Plans -s & ( & $31 / 96$ & 5118100 & & $1 \%$ & $\ldots . .$. & & & & & & & & & \\
\hline 31140.1 & Completo, Mainlenance $\&$ Overthau Plan - 5 & . & (11115/97 & {$[1 / 1 / 15 / 97$} & 0.25 & $0 \%$ & 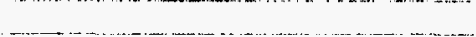 & & & & & & & & & \\
\hline 311402 & Complete, Field Senvice Plan - $S$ & & $12117 / 98$ & $212117 / 98$ & 0.25 & $0 \%$ & $\ldots$ & & & & & & & & & \\
\hline 31140.3 & Complele, Warranty Plan - 5 & $\ldots$ & 1217798 & $12 / 17 / 98$ & 0.25 & $0 \%$. & 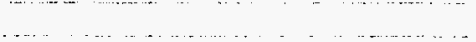 & & & & & & & & & \\
\hline 31140.4 & Complete, Extended Warrany Plan - $\bar{s}$ & & 4127100 & 427100 & 0.25 & $0 \%$ & 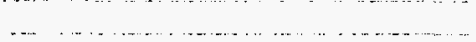 & & & & & & $\Delta$ & & & \\
\hline 31200 & Training $-\mathrm{s}$ & & 71198 & S1211001 & & 1\%) & 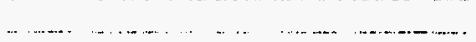 & & & & & & & & & \\
\hline 31200.1 & Complele First Drant Information Handbook - $s$ & & $10 / 15 / 97$ & $10 / 15 / 87$ & 0.10 & $0 \%$ & 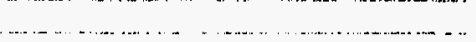 & & & th & & & & & & \\
\hline 31200.2 & Publish Intormation Handbook $-\mathrm{S}$ & & $11117 / 197$ & $11117 / 79$ & 0.15 & $0 \%$ & & & & & & & & & & \\
\hline 31200.3 & Complete Training Plan -s & & $3 / 20 / 98$ & $3 / 20198$ & 0.15 & $0 \%$ & $\ldots$ & & & & $\Delta$ & & & & & \\
\hline 31200.4 & Complete Course Material - $\mathrm{s}$ & & $338 / 99$ & 3//9/99 & 0.15 & $0 \%$ & (n) & & & & & $\Delta$ & & & & \\
\hline 31200.5 & Begin instructional Classes - $\mathrm{s}$ & & $6 / 22100$ & $6 / 22100$ & $0.10^{\circ}$ & $0 \%$ & & & & & & & $\Delta$ & & & \\
\hline 31200.6 & Complote Training-s & & $5 / 21 / 01$ & $5 / 21101$ & 0.35 & $0 \%$. & $\ldots \ldots$ & & & & & & & $\Delta$ & & \\
\hline 31300 & Product Support Capital lema-s & 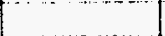 & $111199 / 99$ & $3 / 28101$ & of & $0 \%$ & (n.......... & & & & & & & & & \\
\hline 31300.1 & Complete, Overtacul Tooling - $\mathrm{s}$ & & $12 / 11100$ & $12 / 11100$ & 0.30 & $0 \%$ & (n)................... & & & & & & $\Delta$ & & & \\
\hline 313002 & Complete, Producl Support Upgrades - $\mathrm{S}$ & & 3/28/01 & $3 / 28 / 01$ & 0.35 & $0 \%$ & . & & & & & & & $\Delta$ & & \\
\hline 31300.3 & Complete, Produci Support Equipment - $\mathrm{s}$ & & $3 / 28 / 01$ & $3 / 28 / 01$ & 0.35 & $0 \%$ & - & & & & & & & $\Delta$ & & \\
\hline 32000 & Product Support - L & & 1/5/98 & $12 / 5 / 02$ & & $0 \%$ & $\ldots$ & & & & & & & & & \\
\hline 32100 & Product Support Planning - $L$ & & $1 / 5198$ & $11 / 29 / 02$ & & $0 \%$ & (n............. & & & & & & & & & \\
\hline 32110 & Product Support Plan - $L$ & & isises & $6 / 23 / 99$ & & $0 \%$ & (n)................ & & & & & & & & & \\
\hline 32110.1 & Complete, Product Support Plan - L & & $6 / 23199$ & $6 / 233 / 99$ & 100 & $0 \%$ & & & & & & $\Delta$ & & & & \\
\hline 32120 & Recom. Spare Parrs List - $L$ & & $115 / 98$ & 6/23/99 & of & $0 \%$ & & & & & & $\supseteq$ & & & & \\
\hline 32120.1 & Completel, Recom. Spare Parls Lists - L & & $6 / 23 / 89$ & $8 / 23 / 99$ & 1.00 & $0 \%$ & & & & & & $\Delta$ & & & & \\
\hline 32130 & Customer Services Provissoning Plan $-L$ & & $15 / 98$ & $6 / 23199$ & & $0 \%$ & & & & & & $\square$ & & & & \\
\hline 32130.1 & Complate, Sarvices Provisioning Plan - L & & $6 / 23 / 99$ & $6 / 23 / 99$ & 1.00 & $-0 \%$ & & & & & & $\Delta$ & & & & \\
\hline 32140 & $\begin{array}{l}\text { Sorvices Support Plans - } L \\
\text { Complolo Me Meintenances \& Overthaul Plan - } L\end{array}$ & se & $\begin{array}{l}15 / 98 \\
6 / 23 / 99 \\
6\end{array}$ & \begin{tabular}{|l}
$1 / 29 / 02$ \\
$6 / 23 / 99$
\end{tabular} & $\begin{array}{r}0 \\
025\end{array}$ & $0 \%$ & (................. & & & & & & & & & \\
\hline 32140.1 & $\begin{array}{l}\text { Complele, Mainlenance \& Overhaul Plan }-\mathrm{L} \\
\text { Complete, Field Senvice Plan }-L\end{array}$ & 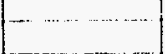 & $\begin{array}{l}6 / 23 / 99 \\
9 / 15 / 100\end{array}$ & $\mid \begin{array}{l}2 / 25399 \\
9 / 15 / 100\end{array}$ & $\begin{array}{r}0.25 \\
-0.25 \\
-0.25\end{array}$ & $0 \%$ & 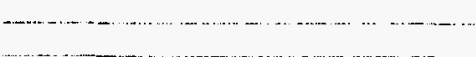 & & & & & $\Delta$ & $\Delta$ & & & \\
\hline 32140.3 & Complete, Warranty Plan - $L$ & $\ldots . . .$. & $9 / 15100$ & $9 / 1500$ & 0.25 & $0 \%$ & & & & & & & $\Delta$ & & & \\
\hline 32140.4 & Complete, Extended Warrenty Plan - L & - & 1129102 & $1 / 29 / 02$ & 0.25 & $0 \%$ & & & & & & & & & & \\
\hline 32200 & Tralining $-\mathrm{L}$ & & 14499 & 3126102 & & $\mathbf{0 \%}$ & $\cdots-$ & & & & & & & & & \\
\hline
\end{tabular}

Printed: 10/30/97

Page 21 
Filter: Total Program

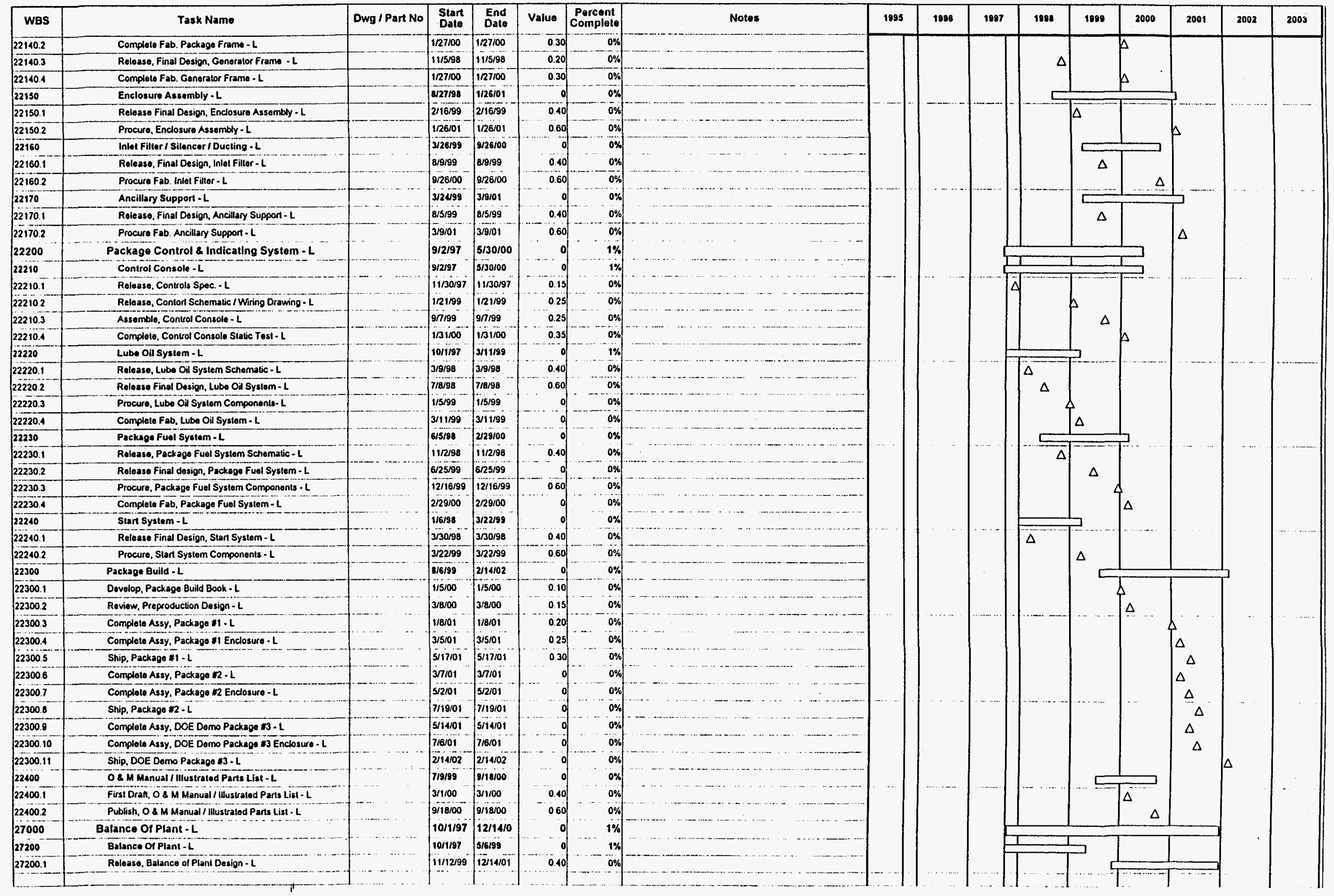

Printed: 10/30/97

Page 20 
Advanced Turbine Systems

Phase 3 and 4

Filter: Total Program

\begin{tabular}{|c|c|c|c|c|c|c|c|c|c|c|c|c|c|c|c|c|}
\hline WBS & Task Name & Dwg / Part No & $\begin{array}{l}\text { Start } \\
\text { Date }\end{array}$ & $\begin{array}{l}\text { End } \\
\text { Date }\end{array}$ & Value & $\begin{array}{l}\text { Porcent } \\
\text { Complete }\end{array}$ & Notes & 1995 & 1998 & 1897 & 1998 & 1989 & 2000 & 2001 & 2002 & $2003^{-}$ \\
\hline 32200.1 & Complete First Oraft, Information Handbook - $\mathrm{L}$ & & $12 / 9 / 99$ & 121999 & 0.10 & $\%$ & 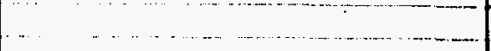 & & & & & & & & & \\
\hline 32200.2 & Complote, Training Plan - $\mathrm{L}$ & & 6,65100 & $6 / 5100$ & 0.15 & $0 \%$ & $\ldots+\ldots-$ & & & & & & $\Delta$ & & & \\
\hline 32200.3 & Publish, Information Handbook - $L$ & & 81100 & B/1100 & 0.15 & $0 \%$ & 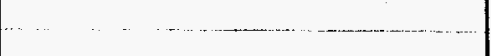 & & & & & & $\Delta$ & & & \\
\hline 32200.4 & & & $5 / 21 / 04$ & $5 / 21 / 01$ & $\begin{array}{r}0.15 \\
-0.10 \\
\end{array}$ & $0 \%$ & 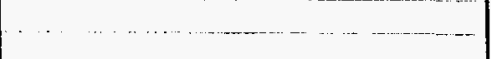 & & & & & & & $\Delta$ & & \\
\hline $\mid \begin{array}{l}32200.5 \\
322008\end{array}$ & $\begin{array}{l}\text { Begin Instruction }-L \\
\text { Complate Training }-L\end{array}$ & $\ldots$ & $9 / 28 / 01$ & $\begin{array}{l}9 / 28 / 01 \\
3 / 26 / 02\end{array}$ & $\begin{array}{l}0.10 \\
0.35\end{array}$ & $0 \%$ & $\ldots \ldots \ldots$ & & & & & & & $\Delta$ & & \\
\hline $\mid \begin{array}{ll}322008 \\
32300\end{array}$ & Product Support Capital Items $-\mathrm{L}$ & & $\begin{array}{l}2 / 2002 \\
3 / 1100\end{array}$ & 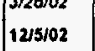 & a. & $0 \%$ & $\left.\begin{array}{llll} & \cdots & \ldots & \ldots\end{array}\right]$ & - & & & & & & & $\Delta$ & \\
\hline 32300.1 & Complote Product Support Upgrados - $i$ & & $12 / 5 / 02$ & $1215 / 02$ & 0.30 & $0 \%$ & . & & & & & & & & & \\
\hline 32300.2 & Complete, Product Suppon Equipment - $L$ & $\cdots n+y$ & [8/30/01 & |8/30/01 & 0.35 & $0 \%$ & 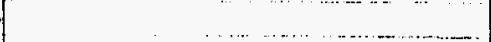 & & & & & & & $\Delta$ & & \\
\hline 32300.3 & Complate, Overtaul Tooling $-\mathrm{L}$ & $\cdots$ & $8 / 30 / 01$ & $8 / 30 / 01$ & 0.35 & $0 \%$ & 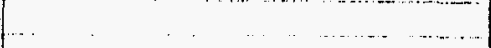 & & & & & & & $\Delta$ & & \\
\hline 40000 & Test \& Evaluation & & 9/14/95 & $\mid \begin{array}{l}9 / 18 / 03 \\
6 / 29 / 01\end{array}$ & & $21 \%$ & $\begin{array}{lllll}\ldots & . & \ldots & & \\
\cdots & \ldots & \end{array}$ & & $1=$ & $=1$ & & & & & & \\
\hline $\begin{array}{l}10100 \\
10100\end{array}$ & $\begin{array}{l}\text { Tost Coordination - } \mathrm{S} \\
\text { Test Coordination }-\mathrm{L}\end{array}$ & $\ldots$ & $\begin{array}{l}9 / 1495 \\
9 / 1 / 97\end{array} \mid$ & 6/2010103 & ? & & $\cdot \cdots+\cdots$ & & & & & & & & & \\
\hline $\begin{array}{l}10100 \\
22000\end{array}$ & Component Testing $\mathrm{s}$ & & $9 / 14 / 95$ & $11 / 21 / 0$ & 0 & $35 \%$ & - -1. & & & & & & & & & \\
\hline $\mid \begin{array}{ll}2000 \\
42100\end{array}$ & Compressor Rig Test - ACE & $\cdots$ & 9/14195 & a/1/197 & 0 & is\% & 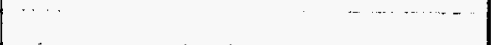 & & & thent & & & & & & \\
\hline 42100.1 & Deliver Design to Agilis, Compressor Rig. ACE & & 10/3/95 & $10 / 3 / 95$ & 0.10 & $100 \%$ & & & & & & & & & & \\
\hline 42100.2 & Complete Design, Compressor Rio-ACE & & 4/101968 & $4 / 1096$ & 0.25 & $100 \%$ & & & $\Delta$ & & & & $\cdots$ & & & \\
\hline$\$ 2100.3$ & Complete Assembly, Compresssor Rig-ACE & & $12 / 30196$ & $12 / 30 / 96$ & 0.25 & $100 \%$ & (2. & & & & & & & & & \\
\hline 42100.4 & Complete Test, Compressor Rig - ACE & 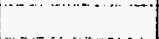 & 73199 & $731 / 97$ & 0.25 & $100 \% \mathrm{~A}$ & Added Tesi Scope & & & A) & & & & & & \\
\hline 42100.5 & Complate Test Repon, Compressor Rig - ACE & & $111 / 1 / 97$ & 1111197 & 0.15 & $0 \%$ & & & & 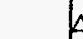 & & & & & & \\
\hline 42200 & Combustor Rig rest.s & & $1 / 16 / 96$ & $3 / 31 / 99$ & 0 & $22 \%$ & & & $\omega_{i+1}+\infty$ & & & & & & & \\
\hline 42210 & ULP Combustion Rig Tost-s & & 1116/96 & $3 / 31 / 98$ & of & $35 \%$ & $=0.0 .+2$ & & {$[x+1$} & & & & & & & \\
\hline 42210.1 & Complete Mod, Single Soctor Combustor Rig - $\mathrm{s}$ & $-2 .-1$ & $6 / 28196$ & $6 / 28 / 96$ & 0.10 & $100 \%$ & 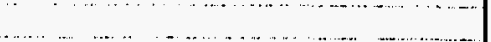 & & $\Delta$ & & & & & & & \\
\hline 42210.2 & Stan Design, ULP Combustor Rig. $\mathrm{S}$ & & $9 / 1 / 96$ & $9 / 1960$ & 0.10 & $100 \%$ & 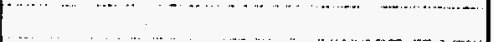 & & $\Delta$ & & & & & & & \\
\hline 42210.2 & Complete Design, ULP Combustor Rig-S & & $4 / 30 / 97$ & $430 / 97$ & 0.15 & $100 \%$ & 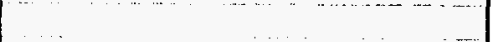 & & & $\Delta$ & & & & & & \\
\hline 122103 & Complete $\mathrm{Fab}$, ULP Combustor Rig - $\mathrm{S}$ & $\ldots .$. & $12110 / 97$ & $12 / 1099$ & 0.15 & $0 \%$ & $\ldots$ & & & & & & & & & \\
\hline 12210.4 & Complete Tests, Single Seclor Combustor Rig - S & 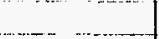 & $10 / 30 / 97$ & 10/30/97 & 0.15 & $0 \%$ & 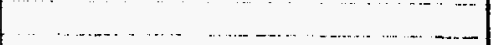 & & & 4 & & & $\cdots$ & & $\cdots$ & \\
\hline 42210.5 & Stant Tesis, ULP Combustor Rig-S & $\ldots \ldots \ldots \ldots$ & $12 / 15 / 97$ & $12115: 97$ & 0.15 & $0 \%$ & $\quad-1$, & & & & & & & & & \\
\hline 42210.6 & Complete Tesis, UtP Combustor Rig-s & $\ldots \ldots \ldots \ldots$ & $3 / 15 / 98$ & $3115 / 98$ & 0.20 & $0 \%$ & $\cdots \quad-1-1-1$ & & & & $\Delta$ & & & & & \\
\hline 42220 & Catalytic Combustion Rig Test - $\mathrm{S}$ & & 12131/96 & $3 / 31 / 98$ & 0 & $10 \%$ & $\ldots+\ldots-1-1-1$ & & & & & & & & & \\
\hline 42220.1 & Start Design, Cataly lic Combustor Rig - $S$ & & $12 / 31 / 96$ & $12 / 31 / 96$ & 0.10 & $100 \%$ & & & & & & & & & & \\
\hline 122220.2 & Complete Design, Catalylic Combustion Rig - $\mathrm{S}$ & & $12 / 31 / 97$ & $12 / 31 / 97$ & 0.15 & $0 \%$ & 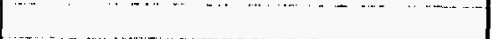 & & & & & & & & & $\ldots$ \\
\hline 42220.3 & Stant Tesis, Calayytic Combuslion Rig - $\mathbf{S}$ & $\ldots$ & $6 / 30 / 98$ & 630198 & 0.10 & $0 \%$ & & & & & $\Delta$ & & & & & \\
\hline 42220.4 & Complete Tests, Catalyuic Combustion Rig $-\bar{S}$ & & $2 / 28 / 99$ & $2 / 28 / 99$ & 0.25 & $0 \%$ & & & & & & $\Delta$ & & & & \\
\hline 422205 & Complete Topical Repor, Catahric Combustion Rig -s & & 3/34/99 & |3/31/99 & & $0 \%$ & 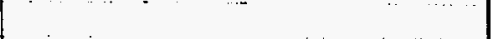 & & & & & $\Delta$ & & & & \\
\hline 12400 & Turtina Rig Teat -S & & sin/196 & $12 / 15 / 97$ & & $35 \%$ & & & Ex+1 & & & & & & & \\
\hline$\$ 2400.1$ & Design to CAT Tech Cir, Turbine Rig - $\mathrm{s}$ & & 6/28/96 & 6/2a/96 & 0.15 & $100 \%$ & & & $\Delta$ & & & $\cdots$ & & & & \\
\hline 42400.2 & Complete Design, Turbine Rig - $\mathbf{s}$ & & 1730197 & $4 / 30 / 97$ & 0.25 & $100 \%[\mathrm{c}$ & Complete except for rodo of stg 182 bleding & & & $\Delta$ & & & & & & \\
\hline 12400.3 & Complete A Assy, Turbine Rig - $\mathrm{S}$ & & $111 / 15 / 97$ & $111 / 15 / 97$ & 0.25 & $0 \% \mathrm{D}$ & Dolayed by design ileralions & & & & & & & & & \\
\hline 42400.4 & Complote Test, Turbine Rig - $\mathrm{S}$ & & $12 / 15 / 97$ & $12 / 15 / 97$ & 0.35 & $0 \%$ & 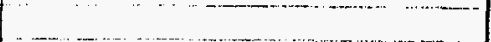 & & & & & & & & & \\
\hline 42500 & Squesezo film Damper Tost - $S$ & & $5 / 10190^{\circ}$ & (1/29/97 & o]. & 50\% & $-\ldots$ & & Har: & & & & & & & \\
\hline 42500.1 & Start Dosign, Squeoze Film Damper Rig - $\mathrm{S}$ & & 51096 & $5110 / 96$ & 0.10 & $100 \%$ & - & & $\Delta$ & & & & & & & \\
\hline$\$ 2500.2$ & Completo Assy, Squaezo Film Damper Rig - $S$ & & 1/30/97 & 430197 & 0.40 & $100 \% \mathrm{~s}$ & Slipped due to adding allemate damper configurations & & & $\Delta$ & & & & & & \\
\hline 22500.3 & Complete Tests, Squeaze Film Damper Rig - $\mathrm{S}$ & 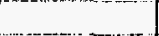 & $11 / 15 / 97$ & $11 / 1199$ & 0.50 & $0 \% \mathrm{~A}$ & Allernate Design being lested. & & & & & & & & & \\
\hline 42600 & In House Engina Test . $\mathrm{s}$ & & 6 & $11 / 21100$ & of & $1 \%$ & & & & & & & & & & \\
\hline 42600.1 & Stan Build, Engino * 1.s & & $3 / 31 / 98$ & $3 / 31 / 98$ & 0.05 & $0 \%$ & 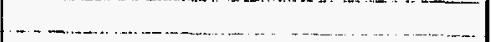 & & & & $\Delta$ & & & & & \\
\hline 42600.2 & Stant Dev Test. Engines $\# 182-\mathrm{S}$ & & $5 / 12198$ & $5 / 12 / 98$ & 0.10 & $0 \%$ & (n) & & & & $\Delta$ & $\cdots$ & & & $*$ & \\
\hline 42600.3 & Stan Build, Engino * 2 (DOE) - 5 & & $5 / 12298$ & $5 / 12 / 98$ & 0.10 & $0 \%$ & $(\ldots \ldots \ldots+\ldots$ & & & & $\Delta$ & & & & & \\
\hline 42600.4 & Complete, Engine \& 2 (DOE) Tost - 5 & & 10/31/98 & $10 / 31 / 98$ & 0.25 & $0 \%$ & $-\ldots+\ldots-1-1-1$ & & & & & & & & & \\
\hline
\end{tabular}

Printed: 10/30/97

Page 22 
Advanced Turbine Systems

Phase 3 and 4

Filter: Total Program

\begin{tabular}{|c|c|c|c|c|c|c|c|c|c|c|c|c|c|c|c|c|}
\hline WBS & Task Name & Dwg / Part No & $\begin{array}{l}\text { Start } \\
\text { Date }\end{array}$ & $\begin{array}{l}\text { End } \\
\text { Date }\end{array}$ & Value & $\begin{array}{c}\text { Percent } \\
\text { Complete }\end{array}$ & Notes & 1995 & 1996 & 1997 & 1998 & 1999 & 2000 & 2001 & 2002 & 2003 \\
\hline 2600.5 & Complate, Engine $* 1$ ULP Test - $\mathrm{s}$ & & 411000 & $4 / 10100$ & 0.25 & $\%$ & 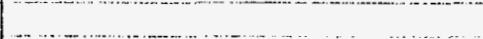 & & & & & & $\Delta$ & & & \\
\hline 42600.6 & Complate, Engine o 1 Cotalytic Test - $\mathrm{s}$ & & $11 / 21100$ & $11 / 2100$ & 0.25 & $0 \%$ & ........... & & & & & & $\Delta$ & & & \\
\hline 12000 & Component Testing $\cdot L$ & & $9 / 30 / 97$ & $3 / 30 / 01$ & 0 & $0 \%$ & 然 & & & & & & & & & \\
\hline 12300 & Combustor Rig Test - $L$ & & $9 / 30 / 97$ & ant1/98 & & $0 \%$ & $\ldots \ldots \ldots$ & & & & & & & & & \\
\hline 42300.1 & Completo Dessign, Combustor Rig - L & & $10 / 20 / 97$ & $10 / 20 / 97$ & & $0 \%$ & $\ldots$ & & & & & & & & & \\
\hline 42300.2 & Comphele Assy, Combustor Rig $\cdot L$ & & $11 / 15198$ & $1115 / 98$ & & $0 \%$ & & & & & $\Delta$ & & & & & \\
\hline 42300.3 & Complete Tessis Combustor Rig $\cdot L$ & & $7 / 14 / 98$ & $7114 / 98$ & & $0 \%$ & $\cdots$ & & & & $\Delta$ & & & & & \\
\hline 42700 & In House Engine Test - $L$ & & 2111100 & 121400 & 0 & $0 \%$ & & & & & & & $E$ & & & \\
\hline 42700.1 & Start Tests, Engine $\| 1-L$ & & 611100 & $611 / 00$ & 0 & $0 \%$ & ......... & & & & & & $\Delta$ & & & \\
\hline 42700.2 & Complete Prod. Test, Engina $* 2-L$ & & $12 / 22100$ & $12 / 22100$ & & $0 \%$ & $\ldots$ & & & & & & & & & \\
\hline 42700.3 & Complete Prod. Test, Engine \#3.L & & $2 / 28 / 01$ & $2 / 28 / 01$ & & $0 \%$ & (n) & & & & & & & $\Delta$ & & \\
\hline 42700.4 & Complete Prod Tesi, DOE Demo Engino * 1 - $\mathrm{L}$ & & $3 / 30101$ & $3 / 30 / 01$ & & $0 \%$ & ....................... & & & & & & & $\Delta$ & & \\
\hline 42800 & Compressor Rlo Test $-L$ & & 61100 & 330101 & & $0 \%$ & (n) & & & & & & & & & \\
\hline 43000 & System Test/Demo-S & & $2 / 13 / 98$ & $11 / 1 / 99$ & & $0 \%$ & 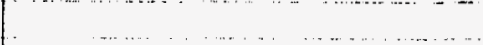 & & & & & & & & & \\
\hline 43100 & Engine C Package Test/Demo -s & & 2113198 & $11 / 1 / 99$ & & $0 \%$ & $\ldots$ & & & & $\square$ & 马 & & & & \\
\hline 43100.1 & Rele ase, Engine Acceptance Test Specification - 5 & & $5 / 5 / 98$ & $5 / 598$ & 0.15 & $0 \%$ & $\ldots$ & & & & $\Delta$ & & & & & \\
\hline 3100.2 & Release. Package Test Specification - $S$ & & 9/9/98 & 9998 & 0.15 & $0 \%$ & 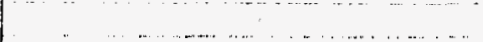 & & & & $\Delta$ & & & & & \\
\hline 431003 & Start Test, Package / Engine * 2 (DOE) - S & & $10 / 5 / 99$ & $1015 / 99^{\circ}$ & 0.30 & $0 \%$ & & & & & & $\Delta$ & & & & \\
\hline 43100.4 & Complete Demo, Package / Engine \& 2 (DDE) - S & & 1111199 & $11 / 1 / 199$ & 0.40 & $0 \%$ & ....... & & & & & $\Delta$ & & & & \\
\hline 43000 & System Test / Demo - L & & $8 / 4 / 00$ & $6 / 21 / 01$ & 0 & $0 \%$ & $\ldots \ldots$ & & & & & & & & & \\
\hline 13200 & Engine E Package Test I Demo - L & & ansoo & $6 / 2101$ & & $0 \%$ & $\ldots$ & & & & & & & & & \\
\hline 43200.1 & Release. Engine Acceptance Test Specificalion - $L$ & & $8 / 31 / 00$ & $8 / 3100$ & 0.15 . & $0 \%$ & $\ldots$ & & & & & & $\Delta$ & & & \\
\hline 43200.2 & Release, Pachage Tesi Specification - L & & 1027100 & $10 / 27 / 00$ & & $0 \%$ & $\ldots \ldots$ & & & & & & $\Delta$ & & & \\
\hline 43200.3 & Slar Test, Package at:-L & & 19/01 & ingro1 & 0.30 . & $0 \%$ & & & & & & & & & & \\
\hline 43200.4 & Complete Acceptance. Package *1 - $L$ & & 2119101 & $2 / 19 / 01$ & o & $0 \%$ & & & & & & & & $\Delta$ & & \\
\hline 432005 & Complete Acceplanco, Package *2 - L & & $4 / 18 / 01$ & $4118 / 01$ & of & $0 \%$ & & & & & & & & $\Delta$ & & \\
\hline 432006 & Complete Accepplance. DOE Demo Package $* 3$ - L & & $6 / 21101$ & $6 / 21101$ & 0.40 & $0 \%$ & 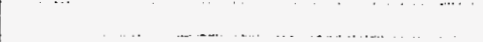 & & & & & & & $\triangle$ & & \\
\hline 44000 & Hosi Site Tesi \& Evaluation -S & & $10 / 1 / 97$ & $6 / 30 / 01$ & & $0 \%$ & & & & & & & & & & \\
\hline 4100 & Host Site Test 8 Evaluation. $-\mathrm{s}$ & & $1011 / 37$ & $6 / 29 / 01$ & \% & $0 \%$ & $\ldots$ & & & & & & & & & \\
\hline 4100.1 & Release, System Tesi Plan - $s$ & & $1 / 27 / 99$ & $1 / 27 / 99$ & 010 & $0 \%$ & ..... & & & & & $\Delta$ & & & & \\
\hline 44100.2 & Deliver Host Sile Senvice Pants - $\mathrm{S}$ & & 212199 & 2/2199 & 0.15 & $0 \%$ & 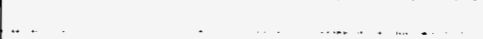 & & & & & $\Delta$ & & & & \\
\hline$\$ 1003$ & Commission, ATS System - $S$ & & $5 / 31 / 00$ & $5 / 31 / 00$ & 0.25 & $0 \%$ & .. & & & & & & $\Delta$ & & & \\
\hline 441004 & Complete, Host Site Evaluation - $\mathbf{s}$ & & $6 / 30101$ & 6/30101 & 045 & $\%$ & $\ldots$ & & & & & & & $\Delta$ & & \\
\hline $\begin{array}{l}4000 \\
44200\end{array}$ & $\begin{array}{c}\text { HosI Site Test \& Evaluation }-L \\
\text { Host Site Test \& Evaluation - } L\end{array}$ & & 9/30/97 & $9 / 18 / 03$ & & $0 \%$ & . . - & & & & & & & & & \\
\hline $\mid \begin{array}{ll}4200 \\
44200.1\end{array}$ & $\begin{array}{l}\text { Host Site Test \& Evaluation - } L \\
\text { Release, System Test Plan - L }\end{array}$ & & $\begin{array}{l}9 / 30 / 91 \\
3 / 27 / 01\end{array}$ & $\begin{array}{l}9 / 18 / 03 \\
3 / 27 / 01\end{array}$ & 0.10 & $0 \%$ & 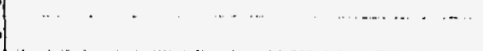 & & & & & & & & & \\
\hline$\left|\begin{array}{l}44200.1 \\
44200.2\end{array}\right|$ & $\begin{array}{l}\text { Release, System Test Plan }-L \\
\text { Deliver Host Site Senvice Part - } L\end{array}$ & & $\begin{array}{l}3 / 27 / 01 \\
8 / 20 / 01\end{array}$ & $\begin{array}{l}3 / 27701 \\
8 / 20 / 01\end{array}$ & 0.15 & $0 \%$ & $\cdots$ & & & & & & & $\Delta$ & & \\
\hline $\begin{array}{l}4200.2 \\
42200.3\end{array}$ & $\begin{array}{l}\text { Deliver Host Site Senvice Part }-L \\
\text { Commission, ATS Sysiem - } L\end{array}$ & & $8 / 14102$ & 8114102 & 0.25 & $0 \%$ & $\cdots$ & & & & & & & & $\Delta$ & \\
\hline 442004 & Complete, Host Sito Evaluation - $L$ & & $8 / 14 / 03$ & $8 / 14 / 03$ & 0.45 & $0 \%$ & - & & & & & & & & & $\Delta$ \\
\hline 50000 & Program Management & & $9 / 14 / 95$ & $8 / 20 / 03$ & 0 & $33 \%$ & & & & & & & & & & \\
\hline 50100 & Program Coordination & $-T_{-1}$ & $9114 / 95$ & $10 / 11101$ & & $33 \%$ & 然 & & & & & & 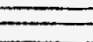 & & & \\
\hline 50200 & Management Rossonve & & 9114195 & $5 \pi 102$ & & $33 \%$ & 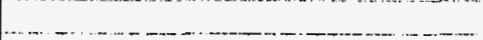 & & & & & - & & $\equiv$ & & \\
\hline 51000 & Customer Present. \& Reports & & $9 / 14 / 95$ & $8 / 20 / 03$ & o) & $33 \%$ & & & & & & & & & & \\
\hline 5 & $\begin{array}{l}\text { Periodic Reports } \\
\text { Special Reports }\end{array}$ & & $\begin{array}{l}\text { g/14/95 } \\
\text { g/14/95 }\end{array}$ & $\mid \begin{array}{l}10 / 1101 \\
0 / 20103\end{array}$ & & $\begin{array}{l}33 \% \\
33 \%\end{array}$ & (n) & & & & & & & & & \\
\hline 51200.1 & Complete, Phase 38 Cosi Proposal & & $12 / 15 / 97$ & $12 / 15 / 97$ & & $0 \%$ & Reschoduled One Year Later, $12 / 96$ & & & & & & & & & \\
\hline 512002 & Completê, Phase 3 A Progresss Report & & $8 / 6 / 98$ & $8 / 6 / 98$ & & $0 \%$ & Rescheduled Ona Yoar Later, $12 / 96$ & & & & $\Delta$ & & & & & \\
\hline 51200.3 & Comprele, Phase 4 Cost Proposal & & $8 / 6 / 99$ & $8 / 6 / 99$ & & $0 \%$ & Reschaduled One Year Later, $12 / 96$ & & & & & $\Delta$ & & & & \\
\hline 51200.4 & Complete, Praso 38 Progress Reporn & & $8 / 3 / 100$ & $9 / 3 / 100$ & & & Roscheduled One Year Later, 12196 & & & & & & & & & \\
\hline
\end{tabular}

Printed: 10/30/97

Page 23 
Advanced Turbine Systems

Phase 3 and 4

Filter: Total Program

\begin{tabular}{|c|c|c|c|c|c|c|c|c|c|c|c|c|c|c|c|c|}
\hline WBS & Task Name & Dwg / Part No & $\begin{array}{l}\text { Start } \\
\text { Date }\end{array}$ & $\begin{array}{l}\text { End } \\
\text { Date }\end{array}$ & Value & \begin{tabular}{c|} 
Percent \\
Complate
\end{tabular} & Notes & 1995 & 1996 & 1897 & 1998 & 1999 & 2000 & 2001 & 2002 & 2003 \\
\hline 51200.5 & Completele, Phase 4 Final Report & & $8 / 20103$ & $8 / 20103$ & o & $0 \%$ & Reschoduleo One Yoar Later, $12 / 96$ & & & & & & & & & $\Delta$ \\
\hline 51300 & Raviewa \& Presentations & & 911495 & 10011101 & of & $33 \%$ & & & & & & & & $\square$ & & \\
\hline 52000 & Project Support & & $9 / 14 / 95$ & $10 / 1 / 01$ & 0 & $33 \%$ & & & & Dif & & & & $\square$ & & \\
\hline $52100^{-1}$ & Business Management & & 911495 & 1011100 & of & $33 \%$ & & & & 然 & & & & $\square$ & & \\
\hline 52200 & Contract Administration & & s/14/95 & $10 / 1 / 101$ & 7 & $33 \%$ & & & & Iin=- & & & & $\square$ & & \\
\hline 52300 & Costischadule Conirol & & O214495 & $10 / 1 / 109$ & 0 & $33 \%$ & & & & 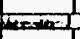 & & & & $\square$ & & \\
\hline $52400^{-}$ & Marketing Coordination & & 911495 & 10/1101 & of & $33 \%$ & & & $E$ & Dit? & & & & $\Longrightarrow$ & & \\
\hline 52500 & Product Cost Management & & grialas & 10/170t & & $33 \%$ & & & 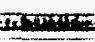 & anes & & & & $\Longrightarrow$ & & \\
\hline 60000 & Commercialization & & 9/14/95 & $5 / 1 / 02$ & o) & $24 \%$ & & & tandsents & iil & & & & & & \\
\hline 61000 & Markei Development & & 911495 & 1011/01 & . & $33 \%$ & & & प्राकात्र & D些 & & & & $\square$ & & \\
\hline 61000.1 & Complete, Market Strategy \& Commercialization Plan & & $3 / 1497$ & $3 / 4<97$ & 0.15 & $100 \%$ & Compleied Draft & & & $\Delta$ & & & & & & \\
\hline 61000.2 & Complete. Market Readiness Plan - Prelim & & 9/30/97 & 9/30/97 & 0.10 & $100 \%$ & & & & & & & & & & \\
\hline 61000.3 & Star, Markel Plan Implemention & & 3/1/99 & 3/1/99 & 0.25 & $0 \%$ & & & & & & $\Delta$ & & & & \\
\hline 610004 & Complete, Market Plan Implementation & & 8/31/01 & $8 / 31 / 01$ & 0.50 & $0 \%$ & & & & & & & & $\Delta$ & & \\
\hline 62000 & Manufacturing Readiness & & 9/14/95 & $5 / 1 / 102$ & o) & $20 \%$ & 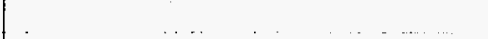 & & & & & & & & & \\
\hline 62100 & Production implementation -s & ..... & 9/14/95 & 9/28/01 & of & $40 \%$ & & & & & & & & & & \\
\hline 621001 & Complate, Production Tooling Plan - $\mathrm{s}$ & & $6 / 14 / 96$ & $6 / 14 / 96$ & 0.10 & $100 \%$ & 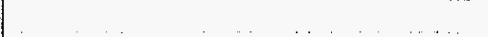 & & $\Delta$ & & & & & & & \\
\hline 621002 & Complete, Production Equipment Requirements - 5 & & $10 / 1796$ & $10 / 17 / 96$ & 0.15 & $100 \%$ & . & & $\Delta$ & & & & & & & \\
\hline 621003 & Complete, Equipment Design \& Facility Requirements -s & & $6 / 16 / 97$ & $6616 / 97$ & 0.15 & $100 \%$ & $\therefore$ & & & $\Delta$ & & & & & & \\
\hline 621004 & Complete Equip Acquisition \& Facilities Rearrange - $s$ & & $5 / 15 / 98$ & 5/15/58 & 0.15 & $0 \%$ & & & & & $\Delta$ & & & & & \\
\hline 621005 & Complete Equip installation, Manpower Acquisition - $S$ & & $5 / 13 / 99$ & $5 / 13 / 99$ & 0.15 & $0 \%$ & . & & & & & $\Delta$ & & & & \\
\hline 621006 & Start Produclion - $\mathrm{s}$ & & $4127 / 100$ & 427700 & 0.15 & $0 \%$ & $\ldots \ldots \ldots$ & & & & & & $\Delta$ & & & \\
\hline 62100.7 & Complote, Production Implementation - $\mathrm{s}$ & & $8 / 30 / 01$ & 8/30/01 & 0.15 & $0 \%$ & $\ldots \ldots$ & & & & & & & $\Delta$ & & \\
\hline 62200 & Production implementation $-L$ & & 9/2/97 & 5/1402 & & $1 \%$ & $\ldots$ & & & & & & & & & \\
\hline 622001 & Complele, Production Tooling Plan- $L$ & & $11 / 15 / 97$ & $\mid 11 / 15 / 97$ & 0.10 & $0 \%$ & 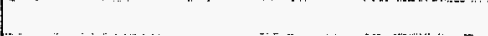 & & & & & & & & & \\
\hline 622002 & Complate. Production Equipment Requiraments - $L$ & & $11 / 15 / 97$ & $11 / 15 / 97$ & 0.15 & $.0 \%$ & . & & & & & & & & & \\
\hline 62200.3 & Complate, Equipment Desion \& Facility Requirements - I & & 3/19/98 & $3 / 199 / 98$ & 0.15 & $0 \%$ & 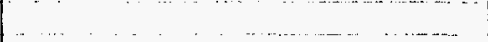 & & & & $\Delta$ & & & & & \\
\hline 622004 & Complete. Equip Acquis ition \& Facilities Rearrango - 1 & $\ldots$ & $2 / 117999$ & $2 / 17199$ & 0.15 & $0 \%$ &. & & & & & $\Delta$ & & & & \\
\hline 62200.5 & Complete, Equip Installation, Manpower Acquisition - $L$ & & $3 / 2100$ & 321200 & 0.15 & $0 \%$ & ............... & & & & & & $\Delta$ & & & \\
\hline 622006 & Slant. Production $-L$ & & 1427701 & 427101 & 0.15 & $0 \%$ & $\ldots \ldots \ldots \ldots$ & & & & & & & $\Delta$ & & \\
\hline 622007 & Complete. Production Implementalion - 1 & & $2 / 28102$ & $2128 / 02$ & 0.15 & $0 \%$ & $\ldots \ldots \ldots-a$ & & & & & & & & $\Delta$ & \\
\hline 63000 & Spin-Off Technology Plan & & 9114/95 & assutot & & $33 \%$ & $\cdots$ & & & & & & & & & \\
\hline 63000.1 & Complete, Preliminary Spin off Technology Plan & & $3 / 15 / 98$ & $3 / 15198$ & 0.40 & $0 \%$ & (n) & & & & $\Delta$ & & & & & \\
\hline 63000.2 & Complete, Final Spin Off Technology Plan & - & $8 / 31 / 01$ & $8 / 31 / 01$ & 0.60 & $0 \%$ & ( & & & & & & & $\Delta$ & & \\
\hline 64000 & Lilo-Cycle Cost Analysis & & 9114/95 & 8/341401 & & $33 \%$ & $\begin{array}{ccccc}\ldots & \ldots & & & \\
\ldots & \ldots & \ldots & \ldots\end{array}$ & & & & & & & & & \\
\hline 65000 & RAMD & & $9 / 1495$ & $8 / 31 / 01$ & & $30 \%$ & 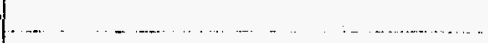 & & & al & & & & & & \\
\hline 65000.1 & Complato, RAMD System Spec & & $8 / 27797$ & $8 / 27 / 97$ & 0.30 & $100 \%$ & ........ & & & $\Delta$ & & & & & & \\
\hline 65000.2 & Complete, RAMD Software \& Hardware Procurement & & $6 / 15 / 98$ & $6 / 15 / 98$ & 0.30 & $0 \%$ & $\ldots$ & & & & $\Delta$ & & & & & \\
\hline 65000.3 & Complete, RAMD Demonsiration & & $8 / 31101$ & $8 / 3101$ & 0.35 & $0 \%$ & . . . . & & & & & & & $\Delta$ & & \\
\hline 70000 & Host Sites & & 9/14/95 & $5 / 2 / 00$ & & $2 \%$ & I. & & & & & & & & & \\
\hline 71000 & Host Site - S & & 9/14/95 & $11 / 1 / 99$ & 0 & $5 \%$ & & & & & & & & & & \\
\hline 71100 & Host Sito Rasearch \& Solection - 5 & & 9/1495 & $5 / 30197$ & of & $100 \%$ & - & & समrex & 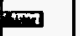 & & & & & & \\
\hline 71100.1 & Complete. Hast Silo Rosoarch - $\mathrm{s}$ & & $9 / 1496$ & $9 / 14 / 96$ & 0.40 & $100 \%$ & & & & & & & & & & \\
\hline 711002 & Complate, Host Site Selection - $\mathrm{s}$ & & $5 / 31 / 97$ & $5 / 31 / 97$ & 0.60 & $100 \%$ & $\ldots \ldots . .$. & & & $\Delta$ & & & & & & \\
\hline 71200 & Host Site Design - S & & 101/1/97. & 1112199 & & $1 \%$ & .......... & & & & & & & & & \\
\hline 72001 & Complate Hosi Site Conversion Plan -s & & $\mid 531 / 98$ & 553199 & 0.40 & $0 \%$ & (n) & & & & $\Delta$ & & & & & \\
\hline 71200.2 & Complate Design, Hosi Site - S & 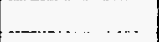 & $11 / 30 / 90$ & $11 / 30 / 98$ & 0.60 & $0 \%$ & 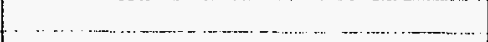 & & & & & & & & & \\
\hline 71300 & Hosi Sile Construction-s & & 10i1/98 & $111 / 1199$ & of & $0 \%$ & 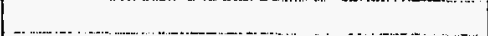 & & & & & & & & & \\
\hline 71300.1 & Complete Construction, Host Sita - $\mathbf{s}$ & & $11 / 1 / 19$ & $11 / 1 / 19$ & 1.00 & $0 \%$ & $\ldots$ & & & & & & & & & \\
\hline
\end{tabular}

Printed: 10/30/97

Page 24 
Advanced Turbine Systems

Phase 3 and 4

Filter: Total Program

\begin{tabular}{|c|c|c|c|c|c|c|c|c|c|c|c|c|c|c|c|c|}
\hline WBS & Task Name & Dwg / Part No & $\begin{array}{l}\text { Stant } \\
\text { Date }\end{array}$ & $\begin{array}{l}\text { End } \\
\text { Date }\end{array}$ & Value & $\begin{array}{c}\text { Percent } \\
\text { Complete }\end{array}$ & Notes & 1995 & 1996 & 1997 & 1988 & 1999 & 2000 & 2001 & 2002 & 2003 \\
\hline 72000 & Host Site $-L$ & & $10 / 1 / 97$ & $5 / 2 / 00$ & & $0 \%$ & & & & & & & $\square$ & & & \\
\hline 72100 & Host Site Research \& Selection - 1 & & 10/1197 & $12 / 18 / 98$ & & $1 \%$ & & & & & & & & & & \\
\hline 72100.1 & Complete, Host Site Research - $L^{-}$ & & S/1/98 & $5 / 1198$ & 0.35 & $0 \%$ & ........ & & & & $\Delta$ & & & & & \\
\hline 72100.2 & Complate, Host Site Selection - $\mathrm{L}$ & & $12 / 18 / 98$ & $12 / 18 / 98$ & 065 & $0 \%$ & $\ldots$ & & .. & & & & & & & \\
\hline 72200 & Host Sile Design - $L$ & & $10 / 2298$ & $10 \pi 199$ & 0 & $0 \%$ & .. .. & & 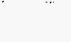 & & $\frac{1}{1}$ & 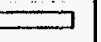 & & & & \\
\hline 72200. & Complete, Host Sile Conversion Plan - $L$ & & 2122199 & $2 / 22199$ & 0.25 & $0 \%$. & & & & & & $\Delta$ & & & & \\
\hline 72200.2 & Complete Design. Host Site $-l$ & & $10 \pi / 99$ & $10 / 799$ & 0.75 & $0 \%$ & 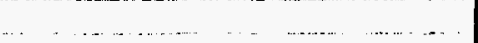 & & & & & $\Delta$ & & & & \\
\hline 72300 & Host Sitt Construction - L & & 1018/99 & 512100 & & $0 \%$ & $\ldots \ldots \ldots$ & & & & & & $\square$ & & & \\
\hline 72300.1 & Complete Consiruction, Host Site - L & & $5 / 2 / 00$ & $5 / 2100$ & 1.00 & $0 \%$ & $\ldots$ & & & & & & $\Delta$ & & & \\
\hline 80000 & Industrial Facilities & & $5 / 1 / 97$ & $7 / 31 / 99$ & 0 & $0 \%$ & 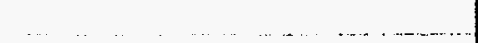 & & & & - & & & & & \\
\hline 81000 & Test Cells - S & $\cdots$ & $5 / 1197$ & $3 / 31 / 99$ & & $1 \%$. & $\ldots$ & & & & & & & & & \\
\hline 810001 & Comploto NR, Tesi Cell Facility - s & & $1211 / 97$ & 1211197 & 0.15 & $0 \%$ & & & & & & & & & & \\
\hline 81000.2 & Complele Pemits, Yost Coll Facility -s & & 1/31/98 & $1 / 31 / 98$ & 025 & $0 \%$ & $\ldots$ & & & & $\Delta$ & & & & & \\
\hline 810003 & Start Construction, Tesi Cell Faciity -s & & $2 / 18 / 98^{\circ}$ & $2118 / 98$ & 0.25 & $0 \%$ & & & & & & & & & & \\
\hline 81000.4 & Complete Checkoul, Test Cell Facility - $\mathbf{S}$ & & $3 / 31 / 99$ & $3 / 31 / 99$ & 0.35 & $0 \%$ & . & & & & & $\Delta$ & & & & \\
\hline 82000 & Test Cells $-\mathrm{L}$ & & rool1997. & $7330 / 99$ & 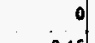 & $0 \%$ & $\ldots . .$. & & & & & & & & & \\
\hline 82000.1 & Complete ANR, Tosi Cell Facility $-\mathrm{L}$ & & $1 / 12,98$ & 1112198 & 0.15 & $0 \%$ & $\ldots \ldots$ & & & & & & & & & \\
\hline 82000.2 & Complete Parmits, Tessi Cell Faciliy $-\mathbf{L}$ & & $5 / 18 / 98$ & $5 / 18 / 98$ & 0.25 & $0 \%$ & $\ldots$ & & & & $\Delta$ & & & & & \\
\hline 82000.3 & Star Construction, Tesi Cell Facilty - L & & $10 / 198$ & $10 / 1 / 98$ & 0.25 & $0 \%$ & $\ldots \ldots \ldots$ & & & & $\Delta$ & & & & & \\
\hline 02000.4 & Complete Checkout. Test Cell Facility $-L$ & & $7 / 31 / 99$ & $7 / 31 / 99$ & 035 & $0 \%$ & $\ldots$ & & & & & $\Delta$ & & & & \\
\hline
\end{tabular}

